\title{
CHARACTER LEVELS AND CHARACTER BOUNDS
}

\author{
ROBERT M. GURALNICK ${ }^{1}$, MICHAEL LARSEN ${ }^{2}$ and PHAM HUU TIEP ${ }^{3}$ \\ ${ }^{1}$ Department of Mathematics, University of Southern California, Los Angeles, CA 90089, USA; \\ email: guralnic@math.usc.edu \\ ${ }^{2}$ Department of Mathematics, Indiana University, Bloomington, IN 47405, USA; \\ email: mjlarsen@indiana.edu \\ ${ }^{3}$ Department of Mathematics, Rutgers University, Piscataway, NJ 08854, USA; \\ email: tiep@math.rutgers.edu
}

Received 30 January 2019; accepted 14 November 2019

\begin{abstract}
We develop the concept of character level for the complex irreducible characters of finite, general or special, linear and unitary groups. We give characterizations of the level of a character in terms of its Lusztig label and in terms of its degree. Then we prove explicit upper bounds for character values at elements with not-too-large centralizers and derive upper bounds on the covering number and mixing time of random walks corresponding to these conjugacy classes. We also characterize the level of the character in terms of certain dual pairs and prove explicit exponential character bounds for the character values, provided that the level is not too large. Several further applications are also provided. Related results for other finite classical groups are obtained in the sequel [Guralnick et al. 'Character levels and character bounds for finite classical groups', Preprint, 2019, arXiv:1904.08070] by different methods.
\end{abstract}

2010 Mathematics Subject Classification: 20C33 (primary); 20C15, 20 P05 (secondary)

\section{Introduction}

It is well known that the complex irreducible characters of the symmetric group $\mathrm{S}_{n}$ are indexed by partitions $\lambda=\left(\lambda_{1}, \ldots, \lambda_{r}\right)$ of $n$. If $\lambda_{2}, \ldots, \lambda_{r}$ are fixed and $\lambda_{1}$ (and therefore $n$ ) goes to infinity, by the hook length formula, the degree of the character $\chi^{\lambda}$ is given by a polynomial in $n$ of degree $d:=\lambda_{2}+\cdots+$ $\lambda_{r}$. For instance, $d=0$ corresponds to the partition $(n)$ and therefore to the trivial character; $d=1$ corresponds to $(n-1,1)$ and therefore to the standard representation of $\mathrm{S}_{n} ; d=2$ corresponds to $(n-2,2)$ and $(n-2,1,1)$, each of

(c) The Author(s) 2020. This is an Open Access article, distributed under the terms of the Creative Commons Attribution licence (http://creativecommons.org/licenses/by/4.0/), which permits unrestricted re-use, distribution, and reproduction in any medium, provided the original work is properly cited. 
degree quadratic in $n$, and each appearing in the tensor square of the standard representation. It makes sense, therefore, to sort the irreducible characters of $\mathrm{S}_{n}$, at least those for which $n$ is large compared to $d$, by their level which is defined to be $d$. If we consider not $\mathrm{S}_{n}$ but $\mathrm{A}_{n}$, then all irreducible characters of $\mathrm{S}_{n}$ of low level compared to $n$ restrict to irreducible characters of $\mathrm{A}_{n}$, and all irreducible characters of $A_{n}$ of low degree arise in this way. Replacing $A_{n}$ by a Schur cover would not change this picture since the minimal degree of a projective representation of $\mathrm{A}_{n}$ which does not lift to a linear representation of $\mathrm{A}_{n}$ grows exponentially in $n$.

Something similar happens for finite simple, or quasisimple, groups of Lie type. For instance, a glance at the list of character degrees of the finite special linear group $G=\mathrm{SL}_{n}(q)$ or of the finite special unitary group $G=\mathrm{SU}_{n}(q)$ reveals that $G$ has one irreducible character of degree 1 ('zero level'), then roughly $q$ characters of degree roughly $q^{n-1}$ ('first level'), then roughly $q^{2}$ characters of degree roughly $q^{2 n-4}$ and $q^{2 n-3}$ ('second level'), and so on. In many applications, it happens that characters of high enough level display a generic behavior and can be handled by uniform arguments, whereas the ones of low level can only be treated individually and after their explicit identification and construction.

But how should one define the level of irreducible characters $\chi \in \operatorname{Irr}(G)$ for a finite group $G$ of Lie type defined over $\mathbb{F}_{q}$ ? There appear to be a few possible approaches, one using the Deligne-Lusztig theory [L], particularly the notions of unipotent support [Geck, GM, L] and wave front sets [K], and another utilizing restrictions to 'nice' subgroups; cf. [GMST, GH1, GH2, GT, T2, TZ]. At present, our attempts in exploring the first approach have yielded strong asymptotic results on character values for the elements $g \in G$ with the property that the centralizer of the semisimple part of $g$ in the underlying algebraic group $\mathcal{G}$ is contained in a proper Levi subgroup of $\mathcal{G}$; see [BLST, TT]. The second approach can lead to more explicit results, but, so far, only for characters of very low (first and, possibly, second) level.

The goal of this paper is to develop the concept of character level for complex irreducible characters of finite, general or special, linear and unitary groups, that is for $\mathrm{GL}_{n}(q), \mathrm{GU}_{n}(q), \mathrm{SL}_{n}(q)$, and $\mathrm{SU}_{n}(q)$, where $q$ is a prime power. We will use the notation $\mathrm{GL}^{\epsilon}$ to denote GL when $\epsilon=+$ and GU when $\epsilon=-$, and similarly for $\mathrm{SL}^{\epsilon}$. For a given $q$ and a given $\epsilon= \pm$, we define $Q:=q$ if $\epsilon=+$ and $Q:=q^{2}$ if $\epsilon=-$. Let $V=\mathbb{F}_{Q}^{n}$ be the natural module of $G \in\left\{\operatorname{GL}_{n}^{\epsilon}(q)\right.$, $\left.\mathrm{SL}_{n}^{\epsilon}(q)\right\}$, where $V$ is endowed with a $G$-invariant nondegenerate Hermitian form if $\epsilon=-$. It is known that the class function

$$
\tau: g \mapsto \epsilon^{n}(\epsilon q)^{\operatorname{dim}_{\mathbb{F}} \operatorname{Ker}\left(g-1_{V}\right)}
$$


is actually a (reducible) character of $G$ (in fact, it is a sum of irreducible Weil characters of $G$, see, for example, $[\mathrm{Ge}, \mathbf{H w}]$, which are finite analogues of the representations constructed in $[\mathrm{W}])$. The true level $\mathfrak{r}^{*}(\chi)$ of an irreducible character $\chi \in \operatorname{Irr}(G)$ is then defined to be the smallest nonnegative integer $j$ such that $\chi$ is an irreducible constituent of $\tau^{j}$, and the level $\mathfrak{l}(\chi)$ is the smallest nonnegative integer $j$ such that $\lambda \chi$ is an irreducible constituent of $\tau^{j}$ for some character $\lambda$ of degree 1 of $G$.

For any, not necessarily irreducible, character $\varphi$ of $G$, the true level $\mathfrak{I}^{*}(\varphi)$ is defined to be the largest true level of its irreducible constituents, and similarly for the level $\mathfrak{l}(\varphi)$. We also define $\mathfrak{l}^{*}(\varphi)=\mathfrak{l}(\varphi)=-\infty$ if $\varphi=0$.

In both the cases $\epsilon= \pm$, we can find a Frobenius endomorphism $F: \mathcal{G} \rightarrow \mathcal{G}$ on the reductive algebraic group $\mathcal{G}=\mathrm{GL}_{n}\left(\overline{\mathbb{F}}_{p}\right)$ such that $\mathcal{G}^{F} \cong G_{n}=\mathrm{GL}_{n}^{\epsilon}(q)$. Moreover, given any $1 \leqslant j \leqslant n$, we can find a (not necessarily $F$-stable) parabolic subgroup $\mathcal{P}$ of $\mathcal{G}$ with an $F$-stable Levi subgroup $\mathcal{L}$ such that $\mathcal{L}^{F} \cong$ $G_{j} \times G_{n-j}=\mathrm{GL}_{j}^{\epsilon}(q) \times \mathrm{GL}_{n-j}^{\epsilon}(q)$. In this case, we will denote the Lusztig functors $R_{\mathcal{L} \subset \mathcal{P}}^{\mathcal{G}}$ and ${ }^{*} R_{\mathcal{L} \subset \mathcal{P}}^{\mathcal{G}}$ [DM2, Section 11] of induction and restriction by $R_{G_{j} \times G_{n-j}}^{G_{n}}$ and ${ }^{*} R_{G_{j} \times G_{n-j}}^{G_{n}}$, respectively.

Our first main result, Theorem 3.9, completely determines irreducible characters of $G$ of any given level $j$ in terms of their Lusztig labels. We also give another characterization of characters of level $j$ in terms of certain dual pairs $[\mathrm{Hw}]$.

THEOREM 1.1. Let $q$ be any prime power, $\epsilon= \pm, G=G_{n}=\mathrm{GL}^{\epsilon}(A) \cong \mathrm{GL}_{n}^{\epsilon}(q)$ with $A=\mathbb{F}_{Q}^{n}, S=\mathrm{GL}^{\epsilon}(B) \cong \mathrm{GL}_{j}^{\epsilon}(q)$ with $B=\mathbb{F}_{Q}^{j}$ and $1 \leqslant j \leqslant n$. Let $V:=$ $A \otimes_{\mathbb{F}_{Q}} B$, which in the case $\epsilon=-i$ s endowed with the tensor product of the Hermitian forms on $A$ and $B$, and consider the (reducible) Weil character $\tau$ of $\mathrm{GL}^{\epsilon}(V) \cong \mathrm{GL}_{n j}^{\epsilon}(q)$ as defined in (1.1). Via the natural action of $G \times S$ on $V$, we can view $\tau$ as a character of $G \times S$ and decompose

$$
\left.\tau\right|_{G \times S}=\sum_{\alpha \in \operatorname{Irr}(S)} D_{\alpha} \otimes \alpha,
$$

where $D_{\alpha}$ is either 0 or a character of $G$. Then the following statements hold.

(i) The map

$$
\Theta: \chi \mapsto\left({ }^{*} R_{G_{j} \times G_{n-j}}^{G_{n}}(\chi)\right)^{G_{n-j}}
$$

yields a canonical bijection between the two sets

$$
\left\{\theta \in \operatorname{Irr}\left(\mathrm{GL}_{n}^{\epsilon}(q)\right) \mid \mathfrak{l}^{*}(\theta)=j\right\}
$$

and

$$
\left\{v \in \operatorname{Irr}\left(\mathrm{GL}_{j}^{\epsilon}(q)\right) \mid \mathfrak{l}^{*}(v) \geqslant 2 j-n\right\} .
$$


(ii) If $\epsilon=+$, then the following statements hold:

(a) If $\mathfrak{l}^{*}(\alpha)<2 j-n$, then $\mathfrak{I}^{*}\left(D_{\alpha}\right)<j$.

(b) If $\mathfrak{l}^{*}(\alpha) \geqslant 2 j-n$, then there is a unique irreducible constituent $D_{\alpha}^{\circ} \in$ $\operatorname{Irr}(G)$ of true level $j$ of $D_{\alpha}$ such that $\mathfrak{l}^{*}\left(D_{\alpha}-D_{\alpha}^{\circ}\right)<j$.

(c) The map $\alpha \mapsto D_{\alpha}^{\circ}$ is the inverse of the bijection $\Theta$.

(iii) Let $\epsilon=-$. Suppose that $1 \leqslant j \leqslant n / 2$ if $q$ is odd, and $1 \leqslant j \leqslant \sqrt{n-3 / 4}-$ $1 / 2$ if $q$ is even. Then for any $\alpha \in \operatorname{Irr}(S)$, there is a unique irreducible character $D_{\alpha}^{\circ} \in \operatorname{Irr}(G)$ of true level $j$ of $D_{\alpha}$ such that $\mathfrak{l}^{*}\left(D_{\alpha}-D_{\alpha}^{\circ}\right)<j$.

Using this characterization, we then establish the following upper and lower bounds for the degree of characters in terms of their level. These bounds show, in particular, that if $\chi(1)$ is not too large, then $\mathfrak{l}(\chi)$ is $\left\lceil\frac{\log _{q} \chi(1)}{n}\right\rceil$, agreeing with intuitive understanding of the character level.

TheOREM 1.2. Let $n \geqslant 2, \epsilon= \pm$, and $G=\mathrm{GL}_{n}^{\epsilon}(q)$. Set $\kappa_{+}=1$ and $\kappa_{-}=1 / 2$. Let $\chi \in \operatorname{Irr}(G)$ have level $j=\mathfrak{l}(\chi)$. Then the following statements hold:

(i) $\kappa_{\epsilon} q^{j(n-j)} \leqslant \chi(1) \leqslant q^{n j}$.

(ii) If $j \geqslant n / 2$, then

$$
\chi(1)>(9 / 16)(q-1) q^{n^{2} / 4-1}
$$

if $\epsilon=+$, and

$$
\chi(1) \geqslant(q-1) q^{n^{2} / 4-1}
$$

if $\epsilon=-$. In particular, $\chi(1)>q^{n^{2} / 4-2}$ if $\mathfrak{l}(\chi) \geqslant n / 2$.

(iii) If $n \geqslant 7$ and $\left\lceil(1 / n) \log _{q} \chi(1)\right\rceil<\sqrt{n-1}-1$, then

$$
\mathfrak{l}(\chi)=\left\lceil\frac{\log _{q} \chi(1)}{n}\right\rceil .
$$

The next result gives an analogue of Theorem 1.2 for special linear and unitary groups.

THEOREM 1.3. Let $n \geqslant 2, \epsilon= \pm$, and $S=\operatorname{SL}_{n}^{\epsilon}(q)$. Set $\sigma_{+}=1 /(q-1)$ and $\sigma_{-}=$ $1 / 2(q+1)$. Let $\varphi \in \operatorname{Irr}(S)$ have level $j=\mathfrak{l}(\varphi)$. Then the following statements hold:

(i) $\sigma_{\epsilon} q^{j(n-j)} \leqslant \varphi(1) \leqslant q^{n j}$.

(ii) If $j \geqslant n / 2$, then $\varphi(1)>q^{n^{2} / 4-2} /(q-\epsilon) \geqslant(2 / 3) q^{n^{2} / 4-3}$. 
(iii) If $n \geqslant 7$ and $\left\lceil(1 / n) \log _{q} \varphi(1)\right\rceil<\sqrt{n-1}-1$, then

$$
\mathfrak{l}(\varphi)=\left\lceil\frac{\log _{q} \varphi(1)}{n}\right\rceil .
$$

Next we prove exponential bounds for character values at the group elements with not-too-large centralizers. The first bound shows that character values $\chi(g)$ of 'almost regular' elements in $G$ are arbitrarily small exponentially in comparison to $\chi(1)$ as long as $n$ is sufficiently large.

THEOREM 1.4. There is an explicit function $h=h(C, m): \mathbb{R}_{\geqslant 1} \times \mathbb{Z}_{\geqslant 0} \rightarrow \mathbb{R}_{\geqslant 1}$ such that, for any $C \in \mathbb{R}_{\geqslant 1}, m \in \mathbb{Z}_{\geqslant 0}$, the following statement holds. For any prime power $q$, any $\epsilon= \pm$, any $G=\mathrm{GL}_{n}^{\epsilon}(q)$ or $\mathrm{SL}_{n}^{\epsilon}(q)$ with $n \geqslant h(C, m)$, any $\chi \in \operatorname{Irr}(G)$, and any $g \in G$ with $\left|\mathbf{C}_{\mathrm{GL}_{n}^{\epsilon}(q)}(g)\right| \leqslant q^{C n}$,

$$
|\chi(g)| \leqslant \chi(1)^{1 / 2^{m}} \text {. }
$$

The second bound shows that if the centralizer of $g \in G$ is not too large, then the character values $|\chi(g)|$ can be bounded away from $\chi(1)$ exponentially (and explicitly).

THEOREM 1.5. Let $q$ be any prime power and let $G=\mathrm{GL}_{n}^{\epsilon}(q)$ or $\operatorname{SL}_{n}^{\epsilon}(q)$ with $\epsilon= \pm$. Suppose that $g \in G$ satisfies $\left|\mathbf{C}_{\mathrm{GL}_{n}^{\epsilon}(q)}(g)\right| \leqslant q^{n^{2} / 12}$. Then

$$
|\chi(g)| \leqslant \chi(1)^{8 / 9}
$$

for all $\chi \in \operatorname{Irr}(G)$.

Our characterization of characters of level $j$ in terms of dual pairs (Theorem 1.1) allows us to obtain strong (and explicit) exponential character bounds for all elements in the group, provided that $j$ is not large. For any $g \in \mathrm{GL}_{n}(q)=\mathrm{GL}(A)$, let $\delta(g)$ denote the largest dimension of eigenspaces of $g$ on $A \otimes_{\mathbb{F}_{q}} \overline{\mathbb{F}}_{q}$.

THEOREM 1.6. Let $q$ be any prime power, $n \geqslant 1, \epsilon= \pm$, and $G=\operatorname{GL}_{n}^{\epsilon}(q)$. Then the following statements hold for any $\chi \in \operatorname{Irr}(G)$ :

(i) Suppose $\epsilon=+$ and $\mathfrak{l}(\chi) \leqslant \sqrt{(8 n-17) / 12}-1 / 2$. Then

$$
|\chi(g)|<1.76 \chi(1)^{1-1 / n}
$$

for all $g \in G \backslash \mathbf{Z}(G)$. Moreover, if $\mathfrak{l}(\chi) \leqslant(\sqrt{12 n-59}-1) / 6$, then

$$
|\chi(g)|<1.76 \chi(1)^{\max (1-1 / 2 \mathrm{l}(\chi), \delta(g) / n)}
$$

for all $g \in G$. 
(ii) The same statements as in (i) hold if we replace $G$ by $\mathrm{SL}_{n}(q)$.

(iii) Suppose $\epsilon=-$ and $\mathfrak{l}(\chi) \leqslant \sqrt{n-3 / 4}-1 / 2$. Then

$$
|\chi(g)|<2.43 \chi(1)^{1-1 / n}
$$

for all $g \in G \backslash \mathbf{Z}(G)$. Moreover, if $\mathfrak{l}(\chi) \leqslant \sqrt{n / 2-1}$, then

$$
|\chi(g)|<2.43 \chi(1)^{\max (1-1 / 2 \mathfrak{l}(\chi), \delta(g) / n)}
$$

for all $g \in G$.

(iv) The same statements as in (iii) hold if we replace $G$ by $\mathrm{SU}_{n}(q)$.

The character bounds in Theorems 1.4-1.6 have already proved to be useful in various applications (see, for example, Theorem 1.7 and [LST]). Note that the exponent $1-1 / n$ in the character bounds in Theorem 1.6 is optimal; see Example 9.6(i). Similarly, if $g \in \mathrm{GL}_{n}(q)$, respectively $g \in \mathrm{GU}_{n}(q)$ is close to be a scalar matrix, that is, $\delta(g)$ is very close to $n$, then the exponent $\max (1-1 / 2 \mathfrak{l}(\chi), \delta(g) / n)$ in Theorem 1.6 is again optimal; see Example 9.6(ii). Furthermore, the bijections in Theorem 1.1 are explicitly described (in terms of character labels) in Corollary 3.10. See also Corollary 8.6 for a bijection between irreducible characters of level $j<n / 2$ of $\mathrm{SL}_{n}(q)$, respectively $\mathrm{SU}_{n}(q)$ and irreducible characters of $\mathrm{GL}_{j}(q)$, respectively $\mathrm{GU}_{j}(q)$. These bijections are canonical, see Remark 9.7, and may be helpful in certain situations (for instance, when one would like to control the action of outer automorphisms and Galois automorphisms on irreducible characters).

As an immediate application, we derive upper bounds on the covering number and mixing time of random walks corresponding to certain conjugacy classes in $\mathrm{SL}_{n}^{\epsilon}(q)$.

For a finite group $S$ and a fixed element $g \in S$, consider the conjugacy class $C=g^{S}$ and random walks on the (oriented) Cayley graph $\Gamma(S, C)$ (whose vertices are $x \in S$ and edges are $(x, x h)$ with $x \in S$ and $h \in C)$. Let $P^{t}(x)$ denote the probability that a random product of $t$ conjugates of $g$ is equal to $x \in S$, and let $U(x):=1 /|S|$ denote the uniform probability distribution on $S$. Also, let

$$
\left\|P^{t}-U\right\|_{L^{1}}:=\sum_{x \in S}\left|P^{t}(x)-U(x)\right|, \quad\left\|P^{t}-U\right\|_{L^{\infty}}:=|S| \cdot \max _{x \in S}\left|P^{t}(x)-U(x)\right| .
$$

The mixing time of the random walk on $\Gamma(S, C)$ is defined to be the smallest integer $t \geqslant 1$ such that $\left\|P^{t}-U\right\|_{L^{1}}<1 / e$. 
THEOREM 1.7. Let $S=\mathrm{SL}_{n}^{\epsilon}(q)$ with $\epsilon= \pm$. Let $g \in S$ be such that

$$
\left|\mathbf{C}_{\mathrm{GL}_{n}^{\epsilon}(q)}(g)\right| \leqslant q^{n^{2} / 12},
$$

and let $C=g^{S}$.

(i) Suppose $n \geqslant 19$. If $t \geqslant 19$, then $P^{t}$ converges to $U$ in the $\|\cdot\|_{\infty}$-norm when $q \rightarrow \infty$; in particular, the Cayley graph $\Gamma(S, C)$ has diameter at most 19.

(ii) If $n \geqslant 10$, then the mixing time $T(S, C)$ of the random walk on $\Gamma(S, C)$ is at most 10 for $q$ sufficiently large.

We expect our results on character levels and character bounds to be useful in several other applications as well. Recently, various interesting conjectures on almost uniform distribution of the commutator map on simple groups of Lie type have been raised. Theorem 1.6 has been used in [ST] to refute one of these conjectures, and evidence in support of another, stated by Avni and Shalev [ST, Conjecture 1.7], is provided by our next result, Corollary 7.5. More recently, Theorem 1.4 has been used in [LST] to study probabilistic Waring problems on finite simple groups. As another application, Theorem 4.6 gives a decomposition of the restriction of the Weil representation to a dual pair $\operatorname{GL}_{m}^{\epsilon}(q) \times \operatorname{GL}_{n}^{\epsilon}(q)$ - when $q$ is sufficiently large this is one of the main results of [S2]. Next, Theorem 9.5 explicitly determines the $U$-rank (introduced in [GH2]) for any character of $\mathrm{GL}_{n}(q)$, resolving an open problem raised in [GH2, Conjecture 6.3.5] and generalizing the complex case of the main result of [T2]. We also observe a parity phenomenon for the characters of finite unitary groups, see Corollary 4.8.

Our method relies crucially on the Deligne-Lusztig theory [C, DM2]. It also utilizes several features of finite general and unitary groups $G$, including the fact that the centralizer $\mathbf{C}_{\mathcal{G}^{*}}(s)$ of any semisimple element in the underlying (dual) algebraic group $\mathcal{G}^{*}$ (of type $\mathrm{GL}_{n}$ ) is a Levi subgroup, geometric constructions of unipotent representations of $\mathrm{GL}_{n}(q)$, and the Ennola duality providing a bridge between the character theories of $\mathrm{GL}_{n}(q)$ and $\mathrm{GU}_{n}(q)$. These properties allow us to develop a complete character level theory for $\mathrm{GL}_{n}(q)$ and $\mathrm{GU}_{n}(q)$. They do not, however, hold true for the remaining finite classical groups. A different method, which relies on results proved for $\mathrm{GL}_{n}(q)$ in this paper and leads to only a partial character level theory but still suffices to yield exponential character bounds, is used in the sequel [GLT] to handle all these remaining groups.

Our paper is organized as follows. After proving some preliminary results in Section 2, in Section 3, we prove Theorem 3.9 which characterizes the characters of $\mathrm{GL}_{n}^{\epsilon}(q)$ of given level $j$ in terms of their Lusztig level and also produces a bijection between these characters and a subset of irreducible characters of 
$\mathrm{GL}_{j}^{\epsilon}(q)$. These results allow us to characterize characters of a given level in terms of their degree (Theorem 1.2) in Section 5 and to establish various bounds on character values (Theorems 1.4 and 1.5) for $\mathrm{GL}_{n}^{\epsilon}(q)$ in Section 6. Further results concerning the level and the Alvis-Curtis duality are given in Section 4. Extensions of several main results to special linear and special unitary groups, as well as Theorem 1.7, are proved in Section 7. The proofs of Theorems 1.1 and 1.6 are completed in Section 8. Some further results are given in Section 9, including Theorem 9.5 which determines the rank of any character in terms of its level.

Notation. For any finite group $G, \operatorname{Irr}(G)$ denotes the set of complex irreducible characters of $G, \mathbf{r e g}_{G}$ denotes the regular character of $G$ (that is, $\operatorname{reg}_{G}(g)$ equals $|G|$ if $g=1$ and 0 if $1 \neq g \in G$ ), and $1_{G}$ denotes the principal character of $G$. For a subgroup $H$ of a finite group $G$, a class function $\alpha$ of $H$, and class functions $\beta, \gamma$ of $G,\left.\gamma\right|_{H}$ denotes the restriction of $\gamma$ to $H, \operatorname{Ind}_{H}^{G}(\alpha)$ denotes the induced class function on $G$, and $[\beta, \gamma]_{G}$ denotes the usual scalar product of class functions. We will say that $\chi \in \operatorname{Irr}(G)$ is an irreducible constituent of a class function $\alpha$ on $G$ (and that $\alpha$ contains $\chi$ ) if $[\alpha, \chi]_{G} \neq 0$. Slightly abusing the language, in various arguments, we will also consider the zero function on $G$ as a character of $G$. A generalized character of $G$ is a $\mathbb{Z}$-linear combination of irreducible characters of $G$. For finite groups $G$ and $H, \alpha \otimes \beta$ denotes the (outer) tensor product of class functions $\alpha$ on $G$ and $\beta$ on $H$, and similarly for representations. If $\varphi$ is a generalized character of the direct product $G \times H$, then we will let $\varphi^{H}$ denote the generalized character $\sum_{\alpha \in \operatorname{Irr}(G)} a_{\alpha, 1_{H}} \alpha$ if

$$
\varphi=\sum_{\alpha \in \operatorname{Irr}(G), \beta \in \operatorname{Irr}(H)} a_{\alpha, \beta} \alpha \otimes \beta .
$$

Other notation is standard.

\section{Preliminaries}

Recall that the complex irreducible characters of the symmetric group $\mathrm{S}_{n}$ are labeled by partitions $\lambda \vdash n: \chi=\chi^{\lambda}$. In particular, $\chi^{(n)}=1_{\mathrm{S}_{n}}$. As usual, we write $\lambda=\left(\lambda_{1}, \lambda_{2}, \ldots, \lambda_{r}\right) \vdash n$ if $\lambda_{1} \geqslant \lambda_{2} \geqslant \cdots \geqslant \lambda_{r} \geqslant 0$ and $\sum_{i=1}^{r} \lambda_{i}=n$.

LEMMA 2.1. Consider $\boldsymbol{\gamma}=\left(\gamma_{1}, \gamma_{2}, \ldots, \gamma_{r}\right) \vdash n+m$ and the Young subgroup $Y=\mathrm{S}_{n} \times \mathrm{S}_{m}$ of $S:=\mathrm{S}_{n+m}$ for some $m, n \geqslant 1$.

(i) $\chi^{\gamma}$ can occur in $\operatorname{Ind}_{Y}^{S}\left(\chi^{\alpha} \otimes \chi^{(m)}\right)$ for some $\boldsymbol{\alpha} \vdash n$ if and only if $\gamma_{1} \geqslant m$.

(ii) If $\gamma_{1}=m$, then $\chi^{\gamma}$ occurs in $\operatorname{Ind}_{Y}^{S}\left(\chi^{\alpha} \otimes \chi^{(m)}\right)$ exactly when $\boldsymbol{\alpha}=\left(\gamma_{2}, \gamma_{3}\right.$, $\left.\ldots, \gamma_{r}\right)$, in which case it occurs with multiplicity one. 
Proof. According to Young's rule [JK, 2.8.2], $\chi^{\gamma}$ can occur in $\operatorname{Ind}_{Y}^{S}\left(\chi^{\alpha} \otimes \chi^{(m)}\right)$ for $\boldsymbol{\alpha}=\left(\alpha_{1}, \alpha_{2}, \ldots, \alpha_{r}\right) \vdash n$ if and only if

$$
\alpha_{r} \leqslant \gamma_{r} \leqslant \alpha_{r-1} \leqslant \gamma_{r-1} \leqslant \alpha_{r-2} \leqslant \cdots \leqslant \alpha_{2} \leqslant \gamma_{2} \leqslant \alpha_{1} \leqslant \gamma_{1}
$$

In particular,

$$
n=\sum_{i=1}^{r} \alpha_{i} \geqslant \sum_{i=1}^{r-1} \alpha_{i} \geqslant \sum_{i=2}^{r} \gamma_{i}=(n+m)-\gamma_{1},
$$

and so $\gamma_{1} \geqslant m$. Moreover, if $\gamma_{1}=m$, then we get $\alpha_{r}=0$ and $\boldsymbol{\alpha}=\left(\gamma_{2}, \gamma_{3}, \ldots\right.$, $\left.\gamma_{r}\right)$.

Conversely, suppose that $\gamma_{1} \geqslant m$. Then we can find $r$ integers $t_{1}, t_{2}, \ldots, t_{r} \geqslant 0$ such that $\sum_{i=1}^{r} t_{i}=m$ and

$$
t_{1} \leqslant \gamma_{1}-\gamma_{2}, \quad t_{2} \leqslant \gamma_{2}-\gamma_{3}, \ldots, t_{r-1} \leqslant \gamma_{r-1}-\gamma_{r}, t_{r} \leqslant \gamma_{r} .
$$

Setting $\alpha_{i}=\gamma_{i}-t_{i}$, we see that $\boldsymbol{\alpha}=\left(\alpha_{1}, \alpha_{2}, \ldots, \alpha_{r}\right) \vdash n$ and $\boldsymbol{\alpha}$ satisfies (2.1), and so $\chi^{\gamma}$ occurs in $\operatorname{Ind}_{Y}^{S}\left(\chi^{\alpha} \otimes \chi^{(m)}\right)$. The multiplicity-one claim also follows from Young's rule.

The following well-known observation is essentially due to Brauer.

LEMMA 2.2. Let $\Theta$ be a generalized character of a finite group $G$ which takes exactly $N$ different values $a_{0}=\Theta(1), a_{1}, \ldots, a_{N-1}$ on $G$. Suppose also that $\Theta(g) \neq \Theta(1)$ for all $1 \neq g \in G$. Then every irreducible character $\chi$ of $G$ occurs as an irreducible constituent of $\Theta^{k}$ for some $0 \leqslant k \leqslant N-1$.

Proof. Consider any $\chi \in \operatorname{Irr}(G)$. By assumption,

$$
\left[\chi, \prod_{i=1}^{N-1}\left(\Theta-a_{i} \cdot 1_{G}\right)\right]_{G}=\frac{\chi(1)}{|G|} \prod_{i=1}^{N-1}\left(a_{0}-a_{i}\right) \neq 0,
$$

whence $\left[\chi, \Theta^{k}\right]_{G} \neq 0$ for some $0 \leqslant k \leqslant N-1$.

LEMMA 2.3. Let $G$ be a finite group and let $\mathcal{X}_{0}, \mathcal{X}_{1}, \ldots, \mathcal{X}_{n}$ be $n+1$ disjoint (possibly empty) subsets of $\operatorname{Irr}(G)$. Let $\alpha_{0}, \alpha_{1}, \ldots, \alpha_{n}$ be (not necessarily irreducible) complex characters of $G$ and $\beta_{0}, \beta_{1}, \ldots, \beta_{n}$ be generalized characters of $G$ such that

(a) $\operatorname{Span}_{\mathbb{Z}}\left(\alpha_{0}, \alpha_{1}, \ldots, \alpha_{j}\right)=\operatorname{Span}_{\mathbb{Z}}\left(\beta_{0}, \beta_{1}, \ldots, \beta_{j}\right)$; 
(b) each $\chi \in \mathcal{X}_{j}$ occurs in $\sum_{i=0}^{j} \alpha_{i}$; and

(c) all irreducible constituents of $\beta_{j}$ belong to $\bigcup_{i=0}^{j} \mathcal{X}_{i}$,

for all $0 \leqslant j \leqslant n$. Then for all $0 \leqslant j \leqslant n, \mathcal{X}_{j}$ is precisely the set of irreducible characters of $G$ that occur in $\alpha_{j}$ but not in $\sum_{i=0}^{j-1} \alpha_{i}$.

Proof. We proceed by induction on $0 \leqslant j \leqslant m$. For $j=0$, any $\chi \in \mathcal{X}_{0}$ occurs in $\alpha_{0}$ by (b). Conversely, any irreducible constituent of $\alpha_{0}$ belongs to $\mathcal{X}_{0}$ by (a) and (c).

For the induction step, consider any $\chi \in \mathcal{X}_{j}$. By (b), $\chi$ occurs in $\sum_{i=0}^{j} \alpha_{i}$. If, moreover, $\chi$ occurs in $\sum_{i=0}^{j-1} \alpha_{i}$, then $\chi$ belongs to $\bigcup_{i=0}^{j-1} \mathcal{X}_{i}$ by (a) and (c), a contradiction. Conversely, assume that $\chi \in \operatorname{Irr}(G)$ occurs in $\alpha_{j}$ but not in $\sum_{i=0}^{j-1} \alpha_{i}$. By (a), $\chi$ occurs in $\beta_{i}$ for some $0 \leqslant i \leqslant j$ and so $\chi \in \mathcal{X}_{k}$ for some $0 \leqslant k \leqslant i$ by (c). Hence, $k=j$ by (b), as desired.

LEMMA 2.4. Let $(G, Q)$ be either $\left(\mathrm{GL}_{n}(q), q\right)$ or $\left(\mathrm{GU}_{n}(q), q^{2}\right)$, and let $V=\mathbb{F}_{Q}^{n}$ denote the natural module for $G$. Then, for any $1 \leqslant j \leqslant n$, the number $N_{j}$ of $G$-orbits on the set $\Omega_{j}$ of ordered $j$-tuples $\left(v_{1}, \ldots, v_{j}\right)$ with $v_{i} \in V$ is at most $8 q^{j^{2} / 4}$ in the first case and at most $2 q^{j^{2}}$ in the second case.

Proof. (i) Consider $U=\mathbb{F}_{Q}^{j}$ with a fixed basis $\left(e_{1}, \ldots, e_{j}\right)$. Then there is a natural bijection between $\Omega_{j}$ and $\operatorname{Hom}(U, V)$ : any $\varpi=\left(v_{1}, \ldots, v_{j}\right)$ corresponds to $f=f_{\varpi} \in \operatorname{Hom}(U, V)$ with $f\left(e_{i}\right)=v_{i}$. Suppose that $\varpi^{\prime}=g(\varpi)$ for some $g \in G$. Then $f_{\varpi^{\prime}}=g f_{\varpi}$ and $\operatorname{Ker}\left(f_{\varpi^{\prime}}\right)=\operatorname{Ker}\left(f_{\varpi}\right)$. Moreover, in the case $Q=q^{2}$, the Hermitian forms of $V$ restricted to $f_{\varpi}(V)$ and $f_{\varpi^{\prime}}(V)$ have the same Gram matrices in the bases $\left(f_{\varpi}\left(u_{1}\right), \ldots, f_{\varpi}\left(u_{k}\right)\right)$ and $\left(f_{\varpi^{\prime}}\left(u_{1}\right), \ldots\right.$, $\left.f_{\varpi^{\prime}}\left(u_{k}\right)\right)$, if $\left(u_{1}, \ldots, u_{k}\right)$ is a basis of $U / \operatorname{Ker}\left(f_{\varpi}\right)$. Conversely, assume that $f_{\varpi}$ and $f_{\varpi^{\prime}}$ have the same kernel $W$ for some $\varpi, \varpi^{\prime} \in \Omega_{j}$. Again we fix a basis $\left(u_{1}, \ldots, u_{k}\right)$ of $U / W$. If $Q=q^{2}$, assume, in addition, that the Hermitian forms of $V$ restricted to $f_{\varpi}(V)$ and $f_{\varpi^{\prime}}(V)$ have the same Gram matrices in the bases $\left(f_{\varpi}\left(u_{1}\right), \ldots, f_{\varpi}\left(u_{k}\right)\right)$ and $\left(f_{\varpi^{\prime}}\left(u_{1}\right), \ldots, f_{\varpi^{\prime}}\left(u_{k}\right)\right)$. By Witt's lemma [A, page $81]$, there is some $g \in G$ such that $g\left(f_{\varpi}\left(u_{i}\right)\right)=f_{\varpi^{\prime}}\left(u_{i}\right)$ for all $1 \leqslant i \leqslant k$. Hence, $f_{\varpi^{\prime}}=g f_{\varpi}$ and so $\varpi^{\prime}=g(\varpi)$. Note that there are at most $q^{k^{2}}$ of possibilities for the Gram matrices in the basis $\left(f_{\varpi}\left(u_{1}\right), \ldots, f_{\varpi}\left(u_{k}\right)\right)$.

(ii) Suppose that $Q=q$. Our arguments in (i) show that $N_{j}$ is just the total number of subspaces $W$ in $U$, that is,

$$
N_{j}=\sum_{i=0}^{j}\left(\begin{array}{l}
j \\
i
\end{array}\right)_{q}
$$


where $\left(\begin{array}{l}j \\ i\end{array}\right)_{q}$ denotes the Gaussian binomial coefficient

$$
\left(\begin{array}{l}
j \\
i
\end{array}\right)_{q}:=\frac{\prod_{t=0}^{i-1}\left(q^{j}-q^{t}\right)}{\prod_{t=0}^{i-1}\left(q^{i}-q^{t}\right)}
$$

By [LMT, Lemma 4.1(i)], we have

$$
\left(\begin{array}{l}
j \\
i
\end{array}\right)_{q}=\frac{\prod_{t=0}^{i-1}\left(q^{j}-q^{t}\right)}{\prod_{t=0}^{i-1}\left(q^{i}-q^{t}\right)}=q^{i(j-i)} \frac{\prod_{t=0}^{i-1}\left(1-1 / q^{j-t}\right)}{\prod_{t=0}^{i-1}\left(1-1 / q^{i-t}\right)}<(32 / 9) q^{i(j-i)},
$$

and so $N_{j}<(32 / 9) \sum_{i=0}^{j} q^{i(j-i)}$. Suppose $j=2 j_{0}+1 \geqslant 1$. For $0 \leqslant i \leqslant j$, we can find $0 \leqslant k \leqslant j_{0}$ such that $i \in\left\{j_{0}-k, j_{0}+1+k\right\}$ and $i(j-i)=j_{0}\left(j_{0}+1\right)-k(k+1)$. It follows that

$$
\frac{32}{9} \sum_{i=0}^{j} q^{i(j-i)}<\frac{64}{9} q^{j_{0}\left(j_{0}+1\right)} \sum_{k=0}^{\infty} \frac{1}{q^{k(k+1)}}<8 q^{j^{2} / 4},
$$

yielding the claim. Similarly, if $j=2 j_{0} \geqslant 2$, then writing $i=j_{0} \pm k$ for $0 \leqslant i \leqslant j$, we have $i(j-i)=j_{0}^{2}-k^{2}$, whence

$$
\frac{32}{9} \sum_{i=0}^{j} q^{i(j-i)}<\frac{32}{9} q^{j_{0}^{2}}\left(1+2 \sum_{k=1}^{\infty} \frac{1}{q^{k^{2}}}\right)<8 q^{j^{2} / 4},
$$

and we are done again.

Note that $N_{j} \geqslant q^{\left\lfloor j^{2} / 4\right\rfloor}$ as $G$ has at least $q^{\left\lfloor j^{2} / 4\right\rfloor}$ orbits on $j$-tuples $\varpi$ corresponding to $f_{\varpi}$ with $\operatorname{dim} \operatorname{Ker}\left(f_{\varpi}\right)=\lfloor j / 2\rfloor$.

(iii) Suppose that $Q=q^{2}$. Our arguments in (i) show that $N_{j}$ is at most the sum over $k$ of the total number of $(j-k)$-dimensional subspaces $W$ in $U$ weighted by a factor of $q^{k^{2}}$, that is,

$$
N_{j} \leqslant \sum_{i=0}^{j} q^{(j-i)^{2}}\left(\begin{array}{l}
j \\
i
\end{array}\right)_{Q}=\sum_{i=0}^{j} q^{(j-i)^{2}} \frac{\prod_{t=0}^{i-1}\left(q^{2 j}-q^{2 t}\right)}{\prod_{t=0}^{i-1}\left(q^{2 i}-q^{2 t}\right)} .
$$

For $j-1 \geqslant i \geqslant 1$, by [LMT, Lemma 4.1(i)], we have

$$
\begin{aligned}
& \frac{\prod_{t=0}^{i-1}\left(q^{2 j}-q^{2 t}\right)}{\prod_{t=0}^{i-1}\left(q^{2 i}-q^{2 t}\right)} \\
& \quad=q^{2 i(j-i)} \frac{\prod_{t=0}^{i-1}\left(1-1 / q^{2(j-t)}\right)}{\prod_{t=0}^{i-1}\left(1-1 / q^{2(i-t)}\right)}<\frac{q^{2 i(j-i)}}{1-q^{-2}-q^{-4}+q^{-10}}<(1.46) q^{2 i(j-i)} .
\end{aligned}
$$


It follows that

$$
N_{j}<q^{j^{2}}+(1.46) \sum_{i=1}^{j} q^{j^{2}-i^{2}}<q^{j^{2}}\left(1+(1.46) \sum_{i=1}^{\infty} \frac{1}{q^{i^{2}}}\right)<1.83 q^{j^{2}} .
$$

Note that $N_{j} \geqslant q^{j^{2}}$ if $j \leqslant n / 2$ as $G$ has $q^{j^{2}}$ orbits on linearly independent $j$-tuples, and, in fact, $N_{j}=\prod_{i=1}^{j}\left(q^{2 i-1}+1\right)$ if $1 \leqslant j \leqslant n / 2$, as we show in Corollary 4.7.

Let $\mathcal{G}$ be a connected reductive algebraic group defined over a finite field of characteristic $p$, and let $F: \mathcal{G} \rightarrow \mathcal{G}$ be a Frobenius endomorphism. Let $\mathcal{P}$ be a (not necessarily $F$-stable) parabolic subgroup of $\mathcal{G}$ with a Levi subgroup $\mathcal{L}$ which is $F$-stable. Then the Lusztig induction $R_{\mathcal{L} \subset \mathcal{P}}^{\mathcal{G}}$ is defined and sends generalized characters $\psi$ of $L:=\mathcal{L}^{F}$ to generalized characters of $G:=\mathcal{G}^{F}$; see [DM2, Section 11]. The character formula for $R_{\mathcal{L} \subset \mathcal{P}}^{\mathcal{G}}(\psi)$, see [DM2, Proposition 12.2], utilizes Green functions

$$
Q_{\mathcal{L} \subset \mathcal{P}}^{\mathcal{G}}: \mathcal{G}_{u}^{F} \times \mathcal{L}_{u}^{F} \rightarrow \mathbb{Z}
$$

as defined in [DM2, Definition 12.1], with $\mathcal{G}_{u}^{F}$ and $\mathcal{L}_{u}^{F}$ denoting the set of unipotent elements in $G$ and in $L$. If $\mathcal{P}$ is $F$-stable in addition, then $R_{\mathcal{L} \subset \mathcal{P}}^{\mathcal{G}}$ is just the Harish-Chandra induction $R_{L}^{G}$ (that first inflates any character of $L$ to a character of $P:=\mathcal{P}^{F}$ and then induces to $G$ ). It is known that the Lusztig induction is transitive (see [DM2, Proposition 11.5]); furthermore, it satisfies the 'Mackey formula' in some cases, including the cases where $\mathcal{P}$ is $F$-stable [DM2, Theorem 5.1], or if one of the involved Levi subgroups is a maximal torus [DM2, Proposition 11.3], or if $\mathcal{G}=\mathrm{GL}_{n}(\mathbb{F})$ [DM1, Theorem 2.6]. Moreover, when the Mackey formula holds, it implies that $R_{\mathcal{L} \subset \mathcal{P}}^{\mathcal{G}}$ does not depend on the choice of $\mathcal{P}$ (see [DM2, page 88]). Hence, when we work with $\mathcal{G}$ of type GL, we will therefore write $R_{L}^{G}$ instead of $R_{\mathcal{L} \subset \mathcal{P}}^{\mathcal{G}}$.

We will record some properties of Lusztig induction.

LEMMA 2.5. Let $\mathcal{G}=\mathcal{G}_{1} \times \mathcal{G}_{2}$ be a direct product of connected reductive algebraic groups with a Frobenius endomorphism $F: \mathcal{G} \rightarrow \mathcal{G}$ which stabilizes both $\mathcal{G}_{1}$ and $\mathcal{G}_{2}$. Let $\mathcal{P}_{i}$ be a parabolic subgroup of $\mathcal{G}_{i}$ with an $F$-stable Levi subgroup $\mathcal{L}_{i}$ for $i=1,2$, and let $\mathcal{P}=\mathcal{P}_{1} \times \mathcal{P}_{2}, \mathcal{L}=\mathcal{L}_{1} \times \mathcal{L}_{2}$.

(i) Suppose that $u_{i} \in\left(\mathcal{G}_{i}^{F}\right)_{u}$ and $v_{i} \in\left(\mathcal{L}_{i}^{F}\right)_{u}$ for $i=1,2$. Then

$$
Q_{\mathcal{L} \subset \mathcal{P}}^{\mathcal{G}}\left(u_{1} u_{2}, v_{1} v_{2}\right)=Q_{\mathcal{L}_{1} \subset \mathcal{P}_{1}}^{\mathcal{G}_{1}}\left(u_{1}, v_{1}\right) Q_{\mathcal{L}_{2} \subset \mathcal{P}_{2}}^{\mathcal{G}_{2}}\left(u_{2}, v_{2}\right) .
$$


(ii) Suppose that $\gamma_{i}$ is a generalized character of $\mathcal{L}_{i}^{F}$ for $i=1,2$. Then

$$
R_{\mathcal{L} \subset \mathcal{P}}^{\mathcal{G}}\left(\gamma_{1} \otimes \gamma_{2}\right)=R_{\mathcal{L}_{1} \subset \mathcal{P}_{1}}^{\mathcal{G}_{1}}\left(\gamma_{1}\right) \otimes R_{\mathcal{L}_{2} \subset \mathcal{P}_{2}}^{\mathcal{G}_{2}}\left(\gamma_{2}\right)
$$

Proof. (i) Let $\mathbf{L}: \mathcal{G} \rightarrow \mathcal{G}$ denote the Lang map $\mathbf{L}(g)=g^{-1} F(g)$, and let $\mathcal{U}$ be the unipotent radical of $\mathcal{P}$. Then $(g, l) \in \mathcal{G}^{F} \times \mathcal{L}^{F}$ acts on $\mathbf{L}^{-1}(\mathcal{U})$ via $x \mapsto g x l$, and this turns the $\ell$-adic cohomology group $H_{c}^{j}\left(\mathbf{L}^{-1}(\mathcal{U}), \overline{\mathbb{Q}}_{\ell}\right)$ into a $\mathcal{G}^{F}$-module- $\mathcal{L}^{F}$ for all $j \geqslant 0$, where $\ell \neq p$ is a fixed prime. Next,

$$
Q_{\mathcal{L} \subset \mathcal{P}}^{\mathcal{G}}(u, v)=\frac{1}{\left|\mathcal{L}^{F}\right|} \mathfrak{L}\left((u, v), \mathbf{L}^{-1}(\mathcal{U})\right),
$$

where the Lefschetz number $\mathfrak{L}\left((u, v), \mathbf{L}^{-1}(\mathcal{U})\right)$ is the trace of $(u, v)$ acting on

$$
H_{c}^{*}\left(\mathbf{L}^{-1}(\mathcal{U})\right)=\sum_{j \geqslant 0}(-1)^{j} H_{c}^{j}\left(\mathbf{L}^{-1}(\mathcal{U}), \overline{\mathbb{Q}}_{\ell}\right)
$$

see [DM2, 10.3,12.1].

In our case, $\mathcal{U}=\mathcal{U}_{1} \times \mathcal{U}_{2}$, where $\mathcal{U}_{i}$ is the unipotent radical of $\mathcal{P}_{i}$ for $i=1,2$. It follows that $\mathbf{L}^{-1}(\mathcal{U})=\mathbf{L}^{-1}\left(\mathcal{U}_{1}\right) \times \mathbf{L}^{-1}\left(\mathcal{U}_{2}\right)$, and so

$$
\mathfrak{L}\left(\left(u_{1} u_{2}, v_{1} v_{2}\right), \mathbf{L}^{-1}(\mathcal{U})\right)=\mathfrak{L}\left(\left(u_{1}, v_{1}\right), \mathbf{L}^{-1}\left(\mathcal{U}_{1}\right)\right) \cdot \mathfrak{L}\left(\left(u_{2}, v_{2}\right), \mathbf{L}^{-1}\left(\mathcal{U}_{2}\right)\right)
$$

by [DM2, Proposition 10.9(ii)]. Together with (2.3), this implies the claim.

(ii) By [DM2, Proposition 11.2],

$$
\left(R_{\mathcal{L} \subset \mathcal{P}}^{\mathcal{G}} \gamma\right)(g)=\frac{1}{\left|\mathcal{L}^{F}\right|} \sum_{l \in \mathcal{L}^{F}} \mathfrak{L}\left((g, l), \mathbf{L}^{-1}(\mathcal{U})\right) \gamma\left(l^{-1}\right)
$$

for any generalized character $\gamma$ of $\mathcal{G}^{F}$. Applying this formula to $\gamma:=\gamma_{1} \otimes$ $\gamma_{2}$ and using [DM2, Proposition 10.9(ii)] again (also noting that $\gamma\left((x y)^{-1}\right)=$ $\gamma\left(x^{-1} y^{-1}\right)$ for all $\left.x, y \in \mathcal{G}^{F}\right)$, we obtain the claim.

In view of Lemma 2.5 and the discussion prior to it, when we work with $\mathcal{G}$ a direct product of groups of type GL, we can also write $R_{L}^{G}$ instead of $R_{\mathcal{L} \subset \mathcal{P}}^{\mathcal{G}}$.

COROLLARY 2.6. Let $\mathcal{G}=\mathcal{G}_{1} \times \mathcal{G}_{2}$ be a direct product of connected reductive algebraic groups with a Frobenius endomorphism $F: \mathcal{G} \rightarrow \mathcal{G}$ which stabilizes both $\mathcal{G}_{1}$ and $\mathcal{G}_{2}$. Let $\mathcal{P}_{1}$ be a parabolic subgroup of $\mathcal{G}_{1}$ with an $F$-stable Levi subgroup $\mathcal{L}_{1}$, and let $\mathcal{P}=\mathcal{P}_{1} \times \mathcal{G}_{2}, \mathcal{L}=\mathcal{L}_{1} \times \mathcal{G}_{2}$. Suppose that $\gamma$ is a generalized character of $\mathcal{L}_{1}^{F}$ and $\delta$ is a generalized character of $\mathcal{G}_{2}^{F}$. Then

$$
R_{\mathcal{L} \subset \mathcal{P}}^{\mathcal{G}}(\gamma \otimes \delta)=R_{\mathcal{L}_{1} \subset \mathcal{P}_{1}}^{\mathcal{G}_{1}}(\gamma) \otimes \delta .
$$


Proof. Note that (2.4) applied to $R_{\mathcal{L}_{2} \subset \mathcal{P}_{2}}^{\mathcal{G}_{2}}(\delta)$, where $\mathcal{P}_{2}:=\mathcal{L}_{2}:=\mathcal{G}_{2}$, yields $R_{\mathcal{L}_{2} \subset \mathcal{P}_{2}}^{\mathcal{G}_{2}}(\delta)=\delta$. Now the statement follows by applying Lemma 2.5(ii).

Note that [GKNT, Lemma 2.7(ii)] is a partial case of Corollary 2.6.

\section{Character levels in finite general linear and unitary groups}

In this section, we will characterize the irreducible characters of $\mathrm{GL}_{n}^{\epsilon}(q)$ of given level $j$, in terms of their Lusztig level, and construct a bijection between them and irreducible characters of $\mathrm{GL}_{j}^{\epsilon}(q)$. To this end, in the first two subsections, we will construct some Lusztig induced characters. These characters will play a key role in the subsequent characterizations in the third subsection of characters of given level.

Let $q$ be a prime power, $\epsilon= \pm$, and let $G:=G_{n}:=\mathrm{GL}_{n}^{\epsilon}(q)$ with $n \geqslant 2$, and natural module $V=\left\langle e_{1}, \ldots, e_{n}\right\rangle_{\mathbb{F}_{Q}}$. When $\epsilon=+$, let $\eta_{n}:=\tau_{n}$ denote the permutation character of the action of $G$ on the set of vectors of $V$ so that

$$
\tau_{n}(g)=q^{\operatorname{dim}_{\mathbb{F}_{q}} \operatorname{Ker}\left(g-1_{V}\right)}
$$

for all $g \in G$. When $\epsilon=-$, let $\eta_{n}:=\zeta_{n}$ denote the reducible Weil character of $G$ (see, for example, [Ge, TZ]), that is,

$$
\zeta_{n}(g)=(-1)^{n}(-q)^{\operatorname{dim}_{q^{2}} \operatorname{Ker}\left(g-1_{V}\right)}
$$

for all $g \in G$. Applying Lemma 2.2 to $(G, \Theta)=\left(\operatorname{GL}_{n}^{\epsilon}(q), \eta_{n}\right)$, we get the following.

COROLlaRY 3.1. Each irreducible character of $\mathrm{GL}_{n}^{\epsilon}(q)$ occurs as an irreducible constituent of $\left(\eta_{n}\right)^{k}$ for some $0 \leqslant k \leqslant n$.

In view of Corollary 3.1, we can introduce the following.

DeFinition 1. Let $\chi \in \operatorname{Irr}\left(\operatorname{GL}_{n}^{\epsilon}(q)\right)$.

(i) We say that $\chi$ has true level $j$, and write $\mathfrak{l}^{*}(\chi)=j$, if $j$ is the smallest nonnegative integer such that $\chi$ is an irreducible constituent of $\left(\eta_{n}\right)^{j}$.

(ii) We say that $\chi$ has level $j$, and write $\mathfrak{l}(\chi)=j$, if $j$ is the smallest nonnegative integer such that $\chi \lambda$ is an irreducible constituent of $\left(\eta_{n}\right)^{j}$ for some character $\lambda \in \operatorname{Irr}\left(\mathrm{GL}_{n}^{\epsilon}(q)\right)$ of degree 1 . 
3.1. A Harish-Chandra induced character of $\mathbf{G L}_{\boldsymbol{n}}(\boldsymbol{q})$. Consider the case $\epsilon=+$ so that $G=G_{n}=\mathrm{GL}_{n}(q)$. Fix some $1 \leqslant j \leqslant n-1$ and consider the parabolic subgroup

$$
P=\operatorname{Stab}_{G}\left(\left\langle e_{1}, \ldots, e_{j}\right\rangle_{\mathbb{F}_{q}}\right)
$$

with unipotent radical $U$ and Levi subgroup $L=G_{j} \times G_{n-j}$, where $G_{j}$ is identified with

$$
\bigcap_{i=j+1}^{n} \operatorname{Stab}_{P}\left(e_{i}\right) \cong \operatorname{GL}\left(\left\langle e_{1}, \ldots, e_{j}\right\rangle_{\mathbb{F}_{q}}\right)
$$

and $G_{n-j}$ is identified with

$$
\bigcap_{i=1}^{j} \operatorname{Stab}_{P}\left(e_{i}\right) \cap \operatorname{Stab}_{P}\left(\left\langle e_{j+1}, \ldots, e_{n}\right\rangle_{\mathbb{F}_{q}}\right) \cong \operatorname{GL}\left(\left\langle e_{j+1}, \ldots, e_{n}\right\rangle_{\mathbb{F}_{q}}\right) .
$$

PROPOSITION 3.2. In the above notation,

$$
R_{G_{j} \times G_{n-j}}^{G}\left(\operatorname{reg}_{G_{j}} \otimes 1_{G_{n-j}}\right)=\prod_{i=0}^{j-1}\left(\tau_{n}-q^{i} \cdot 1_{G}\right) .
$$

Proof. Note that $G$ acts transitively on the set $\tilde{\Omega}$ of ordered $j$-tuples $\left(f_{1}, \ldots\right.$, $f_{j}$ ) of linearly independent vectors in $V$, and the corresponding permutation character is

$$
\pi:=\prod_{i=0}^{j-1}\left(\tau_{n}-q^{i} \cdot 1_{G}\right)
$$

Since the stabilizer of $\left(e_{1}, \ldots, e_{j}\right)$ is $H:=U G_{n-j} \triangleleft P$, we have

$$
\pi=\operatorname{Ind}_{H}^{G}\left(1_{H}\right)=\operatorname{Ind}_{P}^{G}(\alpha)=R_{G_{j} \times G_{n-j}}^{G}\left(\operatorname{reg}_{G_{j}} \otimes 1_{G_{n-j}}\right),
$$

where $\alpha$ is trivial at $U$ and equals to

$$
\operatorname{Ind}_{G_{n-j}}^{G_{j} \times G_{n-j}}\left(1_{G_{n-j}}\right)=\operatorname{reg}_{G_{j}} \otimes 1_{G_{n-j}}
$$

as $P / U$-character. Hence, the statement follows.

We use the parametrization of unipotent characters of $\mathrm{GL}_{n}(q)$ by partitions $\lambda \vdash$ $n: \psi=\psi^{\lambda}$, as in [C, Section 13.1]. We will need the following well-known fact about the Harish-Chandra induction of unipotent characters of Levi subgroups of $\mathrm{GL}_{n}(q)$ (see, for example, [J2, (3.5)]). 
LEMMA 3.3. Let $\boldsymbol{\alpha} \vdash m$ and $\boldsymbol{\beta} \vdash n$, and consider the Young subgroup $\mathrm{S}_{m} \times \mathrm{S}_{n}$ of $\mathrm{S}_{m+n}$ and the Levi subgroup $G_{m} \times G_{n}$ of $G_{m+n}=\mathrm{GL}_{m+n}(q)$. Then

$$
\operatorname{Ind}_{\mathrm{S}_{m} \times \mathrm{S}_{n}}^{\mathrm{S}_{m+n}}\left(\chi^{\alpha} \otimes \chi^{\beta}\right)=\sum_{\lambda \vdash m+n} a_{\alpha \beta \lambda} \chi^{\lambda}, \quad R_{G_{m} \times G_{n}}^{G_{m+n}}\left(\psi^{\alpha} \otimes \psi^{\beta}\right)=\sum_{\lambda \vdash m+n} a_{\alpha \beta \lambda} \psi^{\lambda},
$$

where $a_{\alpha \beta \lambda}$ is the Littlewood-Richardson coefficient.

3.2. A Lusztig induced character of $\mathbf{G U}_{n}(\boldsymbol{q})$. In this subsection, we assume $\epsilon=-$ so that $G=G_{n}=\mathrm{GU}_{n}(q)$. We use the parametrization of unipotent characters of $\mathrm{GU}_{n}(q)$ by partitions $\lambda \vdash n: \psi=\psi^{\lambda}$, as in [C, Section 13.1]. We will need the following property of the Lusztig induction of unipotent characters of $\mathrm{GU}_{n}(q)$; see [FS, Proposition (1C)].

LEMMA 3.4. Let $\boldsymbol{\alpha} \vdash m$ and $\boldsymbol{\beta} \vdash n$, and consider the Levi subgroup $G_{m} \times G_{n}$ of $G_{m+n}=\mathrm{GU}_{m+n}(q)$. Then

$$
R_{G_{m} \times G_{n}}^{G_{m+n}}\left(\psi^{\alpha} \otimes \psi^{\beta}\right)=\sum_{\lambda \vdash m+n}\left( \pm a_{\alpha \beta \lambda}\right) \psi^{\lambda},
$$

where $a_{\alpha \beta \lambda}$ is the Littlewood-Richardson coefficient as in Lemma 3.3.

We will now introduce some notation as in [ThV]. Let $F$ act on $\overline{\mathbb{F}}_{q}^{\times}$via $F(x)=$ $x^{-q}$ and let $\Theta$ be the set of $F$-orbits on $\overline{\mathbb{F}}_{q}^{\times}$. Then there is a natural bijection between $\Theta$ and $\Phi$, the set of $F$-irreducible polynomials $f=f(t)$ over $\mathbb{F}_{q^{2}}$, that is, the monic polynomials $f \in \mathbb{F}_{q^{2}}[t]$ for which there is an $F$-orbit $\mathcal{O}$ of length $\operatorname{deg}(f)$ such that $f(t)=\prod_{z \in \mathcal{O}}(f-z)$. Let $\mathcal{P}_{n}$ denote the set of partitions of $n \geqslant 0$, and let $\mathcal{P}=\bigcup_{n=0}^{\infty} \mathcal{P}_{n}$. For $\lambda \in \mathcal{P}_{n}$, we define $|\lambda|=n$. Fix a linear order on $\Phi$. Now, a $\Phi$-partition

$$
\boldsymbol{v}=\left(\boldsymbol{v}\left(f_{1}\right), \boldsymbol{v}\left(f_{2}\right), \boldsymbol{v}\left(f_{3}\right), \ldots\right)
$$

is a sequence of partitions in $\mathcal{P}$ indexed by $\Phi$, of size $\|\boldsymbol{v}\|=$ $\sum_{f \in \mathcal{X}}|\boldsymbol{v}(f)| \operatorname{deg}(f)$. Denote

$$
\mathcal{P}_{n}^{\Phi}=\{\Phi \text {-partitions } \boldsymbol{v} \mid\|\boldsymbol{v}\|=n\}, \quad \mathcal{P}^{\Phi}=\bigcup_{n=1}^{\infty} \mathcal{P}_{n}^{\Phi} .
$$

Then the conjugacy classes $c_{\mu}$ in $\mathrm{GU}_{n}(q)$ are naturally indexed by $\boldsymbol{\mu} \in \mathcal{P}_{n}^{\Phi}$. We let $c_{1}$ denote the class of the identity. Also, for a class $c_{\mu}$, let $\pi_{\mu}$ denote the class function that takes value 1 on $c_{\mu}$ and 0 elsewhere. 
Let $\mathcal{C}_{n}$ denote the space of complex-valued class functions on $\operatorname{GU}_{n}(q)$. Then Ennola defined in [E] the following product $\alpha_{1} \star \alpha_{2} \in \mathcal{C}_{n_{1}+n_{2}}$ for $\alpha_{1} \in \mathcal{C}_{n_{1}}$ and $\alpha_{2} \in \mathcal{C}_{n_{2}}$, where

$$
\alpha_{1} \star \alpha_{2}\left(c_{\lambda}\right)=\sum_{\left\|\mu_{1}\right\|=n_{1},\left\|\mu_{2}\right\|=n_{2}} g_{\boldsymbol{\mu}_{1} \boldsymbol{\mu}_{2}}^{\lambda} \alpha_{1}\left(c_{\boldsymbol{\mu}_{1}}\right) \alpha_{2}\left(c_{\boldsymbol{\mu}_{2}}\right),
$$

and $g_{\mu_{1} \mu_{2}}^{\lambda}$ is defined using the Hall polynomials [M, Ch. II]:

$$
g_{\boldsymbol{\mu}_{1} \boldsymbol{\mu}_{2}}^{\lambda}=\prod_{f \in \Phi} g_{\boldsymbol{\mu}_{1}(f) \boldsymbol{\mu}_{2}(f)}^{\lambda(())}\left((-q)^{\operatorname{deg}(f)}\right) .
$$

It turns out, see [ThV, Corollary 4.2], that $\star$ coincides with the Lusztig induction.

Proposition 3.5. Let $\alpha$ be a class function on $G_{m}=\mathrm{GU}_{m}(q)$ and $\beta$ be a class function on $G_{n}=\operatorname{GU}_{n}(q)$. Then

$$
R_{G_{m} \times G_{n}}^{G_{m+n}}(\alpha \otimes \beta)=\alpha \star \beta .
$$

PROPOSITION 3.6. In the above notation, for $1 \leqslant j \leqslant n-1$, we have

$$
R_{G_{j} \times G_{n-j}}^{G_{n}}\left(\operatorname{reg}_{G_{j}} \otimes 1_{G_{n-j}}\right)=(-1)^{j(n-j)} \prod_{i=0}^{j-1}\left(\zeta_{n}-(-1)^{n-i} q^{i} \cdot 1_{G}\right) .
$$

Proof. Since $\operatorname{reg}_{G_{j}}=\left|G_{j}\right| \pi_{1}$, Proposition 3.5 and (3.3) and (3.4) imply that

$$
\begin{aligned}
R_{G_{j} \times G_{n-j}}^{G_{n}}\left(\operatorname{reg}_{G_{j}} \otimes 1_{G_{n-j}}\right)(g) & =\left|G_{j}\right| \sum_{\|\boldsymbol{v}\|=n-j} g_{\mathbf{1} \boldsymbol{v}}^{\lambda} \\
& =\left|G_{j}\right| \sum_{\|\boldsymbol{v}\|=n-j} \prod_{f \in \Phi} g_{\mathbf{1}(f) \boldsymbol{v}(f)}^{\lambda(f)}\left((-q)^{\operatorname{deg}(f)}\right)
\end{aligned}
$$

if $g \in G_{n}$ belongs to the conjugacy class $c_{\lambda}$. For any prime $r$ and any integer $n$, a finite abelian $r$-group $M$ is said to have type $\lambda=\left(\lambda_{1}, \ldots, \lambda_{k}\right) \vdash n$ if

$$
M \cong C_{r^{\lambda_{1}}} \times C_{r^{\lambda_{2}}} \times \cdots \times C_{r^{\lambda_{k}}} .
$$

Note that the Hall polynomial $g_{\mu \nu}^{\lambda}(x)$ is characterized by the property that, for every prime $r, g_{\mu \nu}^{\lambda}(r)$ is the number of subgroups $N \leqslant M$ such that $N$ has type $\mu$ and $M / N$ has type $\nu$, where $M$ is a fixed abelian $r$-group of type $\lambda$.

Recall that $\mathbf{1}(f)$ equals $\left(1^{j}\right)$ if $f=t-1$ and 0 otherwise. Hence, for any $f \in \Phi$ with $f \neq t-1$, we see that $g_{\mathbf{1}(f) v}^{\lambda}(r)$ equals 1 if $v=\lambda$ and 0 otherwise, 
whence $g_{\mathbf{1}(f) v}^{\lambda}=\delta_{\lambda, v}$. Hence, in the summation in (3.5), we need to consider only the $\boldsymbol{v}$ with $\|\boldsymbol{v}\|=n-j$ and $\boldsymbol{v}(f)=\lambda(f)$ for all $f \neq t-1$, for which

$$
\prod_{f \in \Phi} g_{\mathbf{1}(f) \boldsymbol{v}(f)}^{\lambda(f)}\left((-q)^{\operatorname{deg}(f)}\right)=g_{\left(1^{j}\right), \boldsymbol{v}^{1}}^{\lambda^{1}}(-q),
$$

where $\lambda^{1}:=\lambda(t-1)$ and $\boldsymbol{v}^{1}:=v(t-1)$. Writing $\lambda^{1}=\left(\lambda_{1}, \lambda_{2}, \ldots, \lambda_{k}\right)$ with $k$ nonzero parts, we see that the unipotent part $u$ of $g$ has Jordan canonical form $\operatorname{diag}\left(J_{\lambda_{1}}, J_{\lambda_{2}}, \ldots, J_{\lambda_{k}}\right)$ on $V_{1}=\operatorname{Ker}\left(s-1_{V}\right)$, where $s$ is the semisimple part of $g$ (and $J_{m}$ is the Jordan block of size $m$ with eigenvalue 1). It follows that

$$
k=\operatorname{dim}_{\mathbb{F}_{q^{2}}} \operatorname{Ker}\left(g-1_{V}\right)
$$

First, we consider the case $k<j$. Then $g_{\left(1^{j}\right), v^{1}}^{\lambda^{1}}(r)=0$ for all $r$ (indeed, any abelian $r$-group $M$ of type $\lambda^{1}$ has $r$-rank $k$ and so cannot contain any subgroup $N$ of type $\left(1^{j}\right)$ ), whence $g_{\left(1^{j}\right), v^{1}}^{\lambda^{1}}(-q)=0$, regardless of $\boldsymbol{v}^{1}$. Together with (3.5) and (3.6), this implies that $R_{G_{j} \times G_{n-j}}^{G_{n}}\left(\operatorname{reg}_{G_{j}} \otimes 1_{G_{n-j}}\right)(g)=0$. On the other hand, (3.7) and the condition $k<j$ yield $\prod_{i=0}^{j-1}\left(\zeta_{n}(g)-(-1)^{n-i} q^{i}\right)=0$, and so we are done in this case.

Now we consider the case $k \geqslant j$. Recall that we need to consider only those $\boldsymbol{v}$ with $\boldsymbol{v}(f)=\lambda(f)$ for all $f \neq t-1$, whence $\left|\boldsymbol{v}^{1}\right|=\left|\lambda^{1}\right|-j$. If $M$ is an abelian $r$-group of type $\lambda^{1}$, then it has $r$-rank $k$ and so $\Omega_{1}(M)$ is elementary abelian of rank $k$. Any subgroup $N$ of type $\left(1^{j}\right)$ is then an elementary abelian $r$-subgroup of rank $j$ in $\Omega_{1}(M)$, and $M / N$ has type $v^{\prime}$ for some $v^{\prime} \vdash\left(\left|\lambda^{1}\right|-j\right)$. It follows that $h(r)$ is just the number of elementary abelian subgroups of rank $j$ in $\Omega_{1}(M)$, that is,

$$
h(r)=\frac{\prod_{i=0}^{j-1}\left(r^{k}-r^{i}\right)}{\prod_{i=0}^{j-1}\left(r^{j}-r^{i}\right)},
$$

if we set

$$
h(x):=\sum_{\boldsymbol{v}^{1} \vdash\left(\left|\lambda^{1}\right|-j\right)} g_{\left(1^{j}\right), \boldsymbol{v}^{1}}^{\lambda^{1}}(x) \in \mathbb{C}[x] .
$$

Since this happens for all primes $r$, we can conclude (with using also (3.7)) that

$$
\begin{aligned}
\sum_{\boldsymbol{v}^{1} \vdash\left(\left|\lambda^{1}\right|-j\right)} g_{\left(1^{j}\right), \boldsymbol{v}^{1}}^{\lambda^{1}}(-q) & =h(-q)=\frac{\prod_{i=0}^{j-1}\left((-q)^{k}-(-q)^{i}\right)}{\prod_{i=0}^{j-1}\left((-q)^{j}-(-q)^{i}\right)} \\
& =\frac{\prod_{i=0}^{j-1}\left((-1)^{n} \zeta_{n}(g)-(-q)^{i}\right)}{(-1)^{j^{2}}\left|G_{j}\right|} .
\end{aligned}
$$


Together with (3.5) and (3.6), this implies that

$$
R_{G_{j} \times G_{n-j}}^{G_{n}}\left(\operatorname{reg}_{G_{j}} \otimes 1_{G_{n-j}}\right)(g)=(-1)^{j^{2}} \prod_{i=0}^{j-1}\left((-1)^{n} \zeta_{n}(g)-(-q)^{i}\right),
$$

as stated.

3.3. Characterizations of characters of given level. We will now return to the notation $G=G_{n}=\operatorname{GL}_{n}^{\epsilon}(q)$, with $\epsilon= \pm$ and $q$ any prime power. Let

$$
\omega_{n}:= \begin{cases}\tau_{n}, & \epsilon=+, \\ (-1)^{n} \zeta_{n}, & \epsilon=-.\end{cases}
$$

Then we can combine Propositions 3.2 and 3.6 into a single statement.

Proposition 3.7. For $\epsilon= \pm$ and for any $1 \leqslant j \leqslant n-1$, we have

$$
R_{G_{j} \times G_{n-j}}^{G_{n}}\left(\operatorname{reg}_{G_{j}} \otimes 1_{G_{n-j}}\right)=(-1)^{j} \prod_{i=0}^{j-1}\left(\omega_{n}-(\epsilon q)^{i} \cdot 1_{G}\right) .
$$

We can identify the dual group $G^{*}$ with $G=\mathrm{GL}_{n}^{\epsilon}(q)$ and use Lusztig's classification of complex characters of $G$; see [C, DM2]. If $s \in G$ is a semisimple element, then $\mathcal{E}(G,(s))$ denotes the rational series of irreducible characters of $G$ labeled by the $G$-conjugacy class of $s$. For any semisimple $s \in G$, we can decompose $V=V^{0} \oplus V^{1}$ as direct (orthogonal if $\epsilon=-$ ) sum of $s$-invariant subspaces, where $V^{0}=\bigoplus_{\delta \in \mu_{q-\epsilon 1}} V_{\delta}, s$ acts on $V_{\delta}$ as $\delta \cdot 1_{V_{\delta}}$, and no eigenvalue of $s^{1}:=\left.s\right|_{V^{1}}$ belongs to

$$
\mu_{q-\epsilon 1}:=\left\{x \in \mathbb{F}_{q^{2}}^{\times} \mid x^{q-\epsilon 1}=1\right\} .
$$

Then

$$
\mathbf{C}_{G}(s)=\prod_{\delta \in \mu_{q-\epsilon 1}} \mathrm{GL}^{\epsilon}\left(V_{\delta}\right) \times \mathbf{C}_{\mathrm{GL}^{\epsilon}\left(V^{1}\right)}\left(s^{1}\right) .
$$

Correspondingly, any unipotent character $\psi$ of $\mathbf{C}_{G}(s)$ can be written in the form

$$
\psi=\bigotimes_{\delta \in \mu_{q-\epsilon 1}} \psi^{\gamma_{\delta}} \otimes \psi_{1},
$$

where $\psi^{\gamma_{\delta}}$ is the unipotent character of $\mathrm{GL}^{\epsilon}\left(V_{\delta}\right)$ labeled by a partition $\boldsymbol{\gamma}_{\delta}$ of $\operatorname{dim}_{\mathbb{F}_{Q}} V_{\delta}$, and $\psi_{1}$ is a unipotent character of $\mathbf{C}_{\mathrm{GL}^{\epsilon}\left(V^{1}\right)}\left(s^{1}\right)$. If $V_{\delta}=0$, then we view $\boldsymbol{\gamma}_{\delta}$ as the partition (0) of 0 . (It will follow from Theorem 3.9 that the irreducible 
character labeled by $(s, \psi)$ has true level $n$ if $V_{1}=0$ and level $n$ if $V_{\delta}=0$ for all $\delta \in \mu_{q-\epsilon 1}$.)

Fix an embedding of $\overline{\mathbb{F}}^{\times}$into $\mathbb{C}^{\times}$. Then one can identify $\mathbf{Z}\left(\mathbf{C}_{G}(s)\right)$ with

$$
\operatorname{Hom}\left(\mathbf{C}_{G}(s) /\left[\mathbf{C}_{G}(s), \mathbf{C}_{G}(s)\right], \mathbb{C}^{\times}\right)
$$

as in [FS, (1.16)], and the linear character of $\mathbf{C}_{G}(s)$ corresponding to $s$ will be denoted by $\hat{s}$. Now, the irreducible character $\chi$ of $G$ labeled by $s$ and the unipotent character $\psi$ is

$$
\chi= \pm R_{\mathbf{C}_{G}(s)}^{G}(\hat{s} \psi)
$$

see [FS, page 116].

Proposition 3.8. For $G=\mathrm{GL}_{n}^{\epsilon}(q)$ and $1 \leqslant j \leqslant n$, all irreducible constituents of

$$
\pi:=\prod_{i=0}^{j-1}\left(\omega_{n}-(\epsilon q)^{i} \cdot 1_{G}\right)
$$

are among the characters given in (3.9), where $\psi$ is as in (3.8) and the first part of the partition $\boldsymbol{\gamma}_{1}$ is at least $n-j$. Moreover, if the first part of the partition $\boldsymbol{\gamma}_{1}$ is exactly $n-j$, then the corresponding character is an irreducible constituent of $\pi$.

Proof. (i) Note that the case $j=n$ follows from the proof of Lemma 2.2 applied to $\pi$. So we will assume that $1 \leqslant j \leqslant n-1$. Any $\varphi \in \operatorname{Irr}(G)$ occurs in $\pi$ precisely when it is a constituent of $R_{L}^{G}\left(\operatorname{reg}_{G_{j}} \otimes 1_{G_{n-j}}\right)$ for $L:=G_{j} \times G_{n-j}$, by Proposition 3.7. Thus, there is an irreducible character $\alpha \in \operatorname{Irr}\left(G_{j}\right)$ such that $\varphi$ is an irreducible constituent of $R_{L}^{G}\left(\alpha \otimes 1_{G_{n-j}}\right)$. Now we can find a semisimple element $s=\left(s_{W}, 1_{U}\right) \in G$, where $V=W \oplus U$ (an orthogonal sum if $\epsilon=-$ ), $G_{j}=\mathrm{GL}^{\epsilon}(W), G_{n-j}=\mathrm{GL}^{\epsilon}(U)$, and $\alpha \in \mathcal{E}\left(G_{j},\left(s_{W}\right)\right)$. We also consider the decomposition $W=W^{0} \oplus W^{1}$ for the element $s_{W}$ as prior to (3.8). Then the decomposition $V=V^{0} \oplus V^{1}$ for $s$ satisfies

$$
V^{1}=W^{1}, \quad V_{1}=W_{1} \oplus U, \quad V_{\delta}=W_{\delta}, \quad \forall \delta \in \mu_{q-\epsilon 1} \backslash\{1\} .
$$

Let $\operatorname{dim}_{\mathbb{F}_{Q}} W_{1}=k$ for some $0 \leqslant k \leqslant j$ and $t:=\left.s\right|_{V^{1}}=\left.\left(s_{W}\right)\right|_{W^{1}}$. Now we have

$$
\begin{gathered}
\mathbf{C}_{G_{j}}\left(s_{W}\right)=\mathbf{C}_{\mathrm{GL}^{\epsilon}\left(V^{1}\right)}(t) \times \prod_{1 \neq \delta \in \mu_{q-\epsilon 1}} \mathrm{GL}^{\epsilon}\left(V_{\delta}\right) \times \mathrm{GL}^{\epsilon}\left(W_{1}\right), \\
\mathbf{C}_{G_{n}}(s)=\mathbf{C}_{\mathrm{GL}^{\epsilon}\left(V^{1}\right)}(t) \times \prod_{1 \neq \delta \in \mu_{q-\epsilon 1}} \mathrm{GL}^{\epsilon}\left(V_{\delta}\right) \times \mathrm{GL}^{\epsilon}\left(V_{1}\right) .
\end{gathered}
$$


In what follows, for brevity, we will denote the restriction of $\hat{s}$, the linear character of $\mathbf{C}_{G}(s)$ corresponding to $s$, to any subgroup of $\mathbf{C}_{G}(s)$ by the same symbol $\hat{s}$. In particular, the linear character of $\mathbf{C}_{G_{j}}\left(s_{W}\right)$ corresponding to $s_{W}$ will also be denoted by $\hat{s}$.

According to (3.9) applied to $\alpha$, we have

$$
\alpha= \pm R_{\mathbf{C}_{G_{j}}\left(s_{W}\right)}^{G_{j}}\left(\hat{s}\left(\beta \otimes \psi^{v}\right),\right.
$$

for some unipotent character $\beta$ of $\mathbf{C}_{\mathrm{GL}^{\epsilon}\left(V^{1}\right)}(t) \times \prod_{1 \neq \delta \in \mu_{q-\epsilon 1}} \mathrm{GL}^{\epsilon}\left(V_{\delta}\right) \leqslant G_{j-k}$ and unipotent character $\psi^{\nu}$ of $\mathrm{GL}^{\epsilon}\left(W_{1}\right)=G_{k}$ (so $\boldsymbol{v} \vdash k$ ). Note that $\hat{s}$ is trivial on $\mathrm{GL}^{\epsilon}\left(V_{1}\right)$. By transitivity of the Lusztig induction and Corollary 2.6 (and using [DM2, Proposition 12.6]), we have

$$
\pm \alpha=R_{G_{j-k} \times G_{k}}^{G_{j}}\left(R_{\mathbf{C}_{G_{j}}\left(s_{W}\right)}^{G_{j-k} \times G_{k}}\left(\hat{s} \beta \otimes \psi^{\nu}\right)\right)=R_{G_{j-k} \times G_{k}}^{G_{j}}\left(\tilde{\beta} \otimes \psi^{\nu}\right),
$$

where $\tilde{\beta}:=R_{\mathbf{C}_{G_{j-k}}^{G_{j-k}}\left(s_{W}\right)}(\hat{s} \beta)$. It follows, using the same properties of the Lusztig induction, that

$$
\pm \alpha \otimes 1_{G_{n-j}}=R_{G_{j-k} \times G_{k}}^{G_{j}}\left(\tilde{\beta} \otimes \psi^{\nu}\right) \otimes 1_{G_{n-j}}=R_{G_{j-k} \times G_{k} \times G_{n-j}}^{G_{j} \times G_{n-j}}\left(\tilde{\beta} \otimes \psi^{\nu} \otimes 1_{G_{n-j}}\right) .
$$

Hence,

$$
\begin{aligned}
\pm R_{L}^{G}\left(\alpha \otimes 1_{G_{n-j}}\right) & =R_{G_{j} \times G_{n-j}}^{G_{n}}\left(R_{G_{j-k} \times G_{k} \times G_{n-j}}^{G_{j} \times G_{n-j}}\left(\tilde{\beta} \otimes \psi^{v} \otimes 1_{G_{n-j}}\right)\right) \\
& =R_{G_{j-k} \times G_{k} \times G_{n-j}}^{G_{n}}\left(\tilde{\beta} \otimes \psi^{v} \otimes 1_{G_{n-j}}\right) \\
& =R_{G_{j-k} \times G_{n-j+k}}^{G_{n}}\left(R_{G_{j-k} \times G_{k} \times G_{n-j}}^{G_{j-k} \times G_{n-j k}}\left(\tilde{\beta} \otimes \psi^{v} \otimes 1_{G_{n-j}}\right)\right) \\
& =R_{G_{j-k} \times G_{n-j+k}}^{G_{n}}\left(\tilde{\beta} \otimes R_{G_{k} \times G_{n-j}}^{G_{n-j+k}}\left(\psi^{v} \otimes 1_{G_{n-j}}\right)\right) .
\end{aligned}
$$

By Lemmas 2.1, 3.3, and 3.4, each irreducible constituent of

$$
R_{G_{k} \times G_{n-j}}^{G_{n-j}}\left(\psi^{\nu} \otimes 1_{G_{n-j}}\right)
$$

is $\psi^{\lambda}$ for some $\lambda=\left(\lambda_{1}, \lambda_{2}, \ldots, \lambda_{r}\right) \vdash n-j+k$ with $\lambda_{1} \geqslant n-j$. Again by transitivity of the Lusztig induction [DM2, Proposition 4.7] and Corollary 2.6, we have for any such $\lambda$ that

$$
\begin{aligned}
R_{G_{j-k} \times G_{n-j+k}}^{G_{n}}\left(\tilde{\beta} \otimes \psi^{\lambda}\right) & =R_{G_{j-k} \times G_{n-j+k}}^{G_{n}}\left(R_{\left.\mathbf{C}_{G_{j-k}\left(s_{W}\right)}^{G_{j k}}(\hat{s} \beta) \bigotimes \psi^{\lambda}\right)}\right. \\
& =R_{G_{j-k} \times G_{n-j+k}}^{G_{n}}\left(R_{\mathbf{C}_{G}(s)}^{G_{j-k} \times G_{n-j+k}}\left(\hat{s} \beta \bigotimes \psi^{\lambda}\right)\right) \\
& =R_{\mathbf{C}_{G}(s)}^{G_{n}}\left(\hat{s} \beta \bigotimes \psi^{\lambda}\right) \\
& =R_{\mathbf{C}_{G}(s)}^{G_{n}}\left(\hat{s}\left(\beta \bigotimes \psi^{\lambda}\right)\right),
\end{aligned}
$$


and so by (3.9), it is an irreducible character of $G$ up to sign. We have, therefore, shown that the irreducible constituents of $\pi$ are among the characters given in (3.9) with the first part of the partition $\boldsymbol{\gamma}_{1}=\lambda$ being at least $n-j$.

(ii) Conversely, suppose that the partition $\boldsymbol{\gamma}_{1}=\lambda$ for the character $\varphi$ has the first part $\lambda_{1}=n-j$. The above arguments and Lemmas 2.1, 3.3, and 3.4 show that the multiplicity of $\varphi$ in $R_{L}^{G}\left(\alpha \otimes 1_{G_{n-j}}\right)$ can be nonzero precisely when $\boldsymbol{v}=$ $\left(\lambda_{2}, \lambda_{3}, \ldots, \lambda_{r}\right)$, in which case it is \pm 1 . Certainly, $s$ (up to conjugacy), $\hat{s}$, and $\beta$ are uniquely determined by $\varphi$. Now (3.10) implies that $\alpha$ is uniquely determined by $\varphi$. Thus, there is a unique $\alpha \in \operatorname{Irr}\left(G_{j}\right)$ such that $\varphi$ is an irreducible constituent of $R_{L}^{G}\left(\alpha \otimes 1_{G_{n-j}}\right)$. Using the adjoint functor of Lusztig restriction, we then have

$$
\alpha=\left({ }^{*} R_{G_{j} \times G_{n-j}}^{G_{n}}(\varphi)\right)^{1_{G_{n-j}}} .
$$

Therefore, even though the Lusztig induction $R_{L}^{G}$ may send characters to generalized characters and cancel out irreducible constituents of different $R_{L}^{G}\left(\alpha^{\prime} \otimes 1_{G_{n-j}}\right)$, the established uniqueness of $\alpha$ allows us to conclude that $\varphi$ is an irreducible constituent of $\pi$. Since $\operatorname{reg}_{G_{j}}=\sum_{\alpha^{\prime} \in \operatorname{Irr}\left(G_{j}\right)} \alpha^{\prime}(1) \alpha^{\prime}$, we also see that $[\varphi, \pi]_{G}= \pm \alpha(1)$.

THEOREM 3.9. Let $\varphi$ be an irreducible character of $G=G_{n}=\operatorname{GL}_{n}^{\epsilon}(q)$ which is labeled by the $G$-conjugacy class of a semisimple element $s \in G$ and a unipotent character $\psi$ of $\mathbf{C}_{G}(s)$ written as in (3.8). Let $0 \leqslant j \leqslant n$ be an integer.

(i) $\varphi$ has true level $j$ precisely when the first part of the partition $\boldsymbol{\gamma}_{1}$ is $n-j$. In this case, there is a unique $\alpha \in \operatorname{Irr}\left(G_{j}\right)$ such that $\varphi$ is a constituent of $R_{G_{j} \times G_{n-j}}^{G_{n}}\left(\alpha \otimes 1_{G_{n-j}}\right)$; equivalently,

$$
\alpha=\left({ }^{*} R_{G_{j} \times G_{n-j}}^{G_{n}}(\varphi)\right)^{1_{G_{n-j}}} ;
$$

and $\varphi$ occurs in $\left(\tau_{n}\right)^{j}$ if $\epsilon=+$ and in $\left(\zeta_{n}\right)^{j}$ if $\epsilon=-$ with multiplicity $\alpha(1)$, and $\mathfrak{l}^{*}(\alpha) \geqslant 2 j-n$. Furthermore, the map

$$
\Theta: \varphi \mapsto\left({ }^{*} R_{G_{j} \times G_{n-j}}^{G_{n}}(\varphi)\right)^{1_{G_{n-j}}}
$$

yields a bijection between

$$
\left\{\chi \in \operatorname{Irr}\left(\mathrm{GL}_{n}^{\epsilon}(q)\right) \mid \mathfrak{l}^{*}(\chi)=j\right\}
$$

and

$$
\left\{v \in \operatorname{Irr}\left(\mathrm{GL}_{j}^{\epsilon}(q)\right) \mid \mathfrak{l}^{*}(v) \geqslant 2 j-n\right\} .
$$

(ii) $\varphi$ has level $j$ precisely when the longest among the first parts of the partitions $\boldsymbol{\gamma}_{\delta}, \delta \in \mu_{q-\epsilon 1}$, is $n-j$. 
Proof. (i) For $1 \leqslant i \leqslant n$, define $\alpha_{i}=\left(\tau_{n}\right)^{i}$ when $\epsilon=+$ and $\alpha_{i}=\left(\zeta_{n}\right)^{i}$ when $\epsilon=-$, and let $\beta_{i}:=\prod_{k=0}^{i-1}\left(\omega_{n}-(\epsilon q)^{k} \cdot 1_{G}\right)$. Also, let $\alpha_{0}=\beta_{0}:=1_{G}$. Then condition 2.3(a) is fulfilled. Next, for $0 \leqslant i \leqslant n-1$, let $\mathcal{X}_{i}$ denote the set of irreducible characters $\chi$ labeled by a semisimple element $s \in G$ and a unipotent character $\psi$ of $\mathbf{C}_{G}(s)$ as in (3.8), where the first part of $\boldsymbol{\gamma}_{1}$ is equal to $n-i$, and let $\mathcal{X}_{n}$ denote the set of such irreducible characters $\chi$ where $V_{1}=0$ (that is, $s-1_{V}$ is nondegenerate). Then Proposition 3.8 shows that conditions 2.3(b), (c) are fulfilled. Hence, by Lemma 2.3 and Definition $1, \mathcal{X}_{j}$ is precisely the set of characters of true level $j$.

Suppose now that $I^{*}(\varphi)=j$. Then the existence and uniqueness of $\alpha$ have been established in the proof of Proposition 3.8, where we also showed that $\left[\varphi, \beta_{j}\right]_{G}=$ $\pm \alpha(1)$ and observed (3.11). Note that $\alpha_{j}-(\epsilon 1)^{n j} \beta_{j}$ is a linear combination of $\left(\tau_{n}\right)^{i}, 0 \leqslant i<j$, when $\epsilon=+$, and of $\left(\zeta_{n}\right)^{i}, 0 \leqslant i<j$, when $\epsilon=-$, and so has no constituent of true level $j$ by Definition 1 . Hence,

$$
\left[\varphi, \alpha_{j}\right]_{G}= \pm\left[\varphi, \beta_{j}\right]_{G}= \pm \alpha(1) .
$$

Since $\alpha_{j}$ is a true character, we must have that $\left[\varphi, \alpha_{j}\right]_{G}=\alpha(1)$. Also, in the notation of the proof of Proposition 3.8, $\boldsymbol{\gamma}_{1}=\lambda=\left(n-j, \lambda_{2}, \ldots, \lambda_{r}\right)$ and $\boldsymbol{v}=$ $\left(\lambda_{2}, \ldots, \lambda_{r}\right)$. In particular, $\lambda_{2} \leqslant n-j$, whence $\mathfrak{I}^{*}(\alpha) \geqslant 2 j-n$ by the first statement applied to $G_{j}$, and the map $\Theta$ is well defined. It is injective, as $\varphi$ is uniquely determined by $\alpha$.

Conversely, suppose that $\alpha \in \operatorname{Irr}\left(G_{j}\right)$ as given in (3.10) satisfies $\mathfrak{l}^{*}(\alpha) \geqslant 2 j-n$. Then, in the above notation $\boldsymbol{v}=\left(\lambda_{2}, \ldots, \lambda_{r}\right) \vdash k$, we have $\lambda_{2} \leqslant j-(2 j-n)=$ $n-j$; hence, $\lambda:=\left(n-j, \lambda_{2}, \ldots, \lambda_{r}\right)$ is a partition of $n-j+k$. With $\varphi$ defined as in (3.9), the proof of Proposition 3.8 shows that $\Theta(\varphi)=\alpha$ and so $\Theta$ is onto.

(ii) The linear characters of $G$ are precisely the characters $\hat{t}$ with $t \in \mathbf{Z}(G)$. Also note that if $s \in G$ is semisimple, then $\left.\hat{t}\right|_{\mathbf{C}_{G}(s)} \hat{s}=\widehat{s t}$. Hence, by [DM2, Proposition 12.6], we have $R_{\mathbf{C}_{G}(s)}^{G}(\hat{s} \psi) \hat{t}=R_{\mathbf{C}_{G}(s)}^{G}(\widehat{s t} \psi)$. In particular, if $t=\delta \cdot 1_{V}$ for some $\delta \in \mu_{q-\epsilon 1}$, then the partition $\boldsymbol{\gamma}_{\delta^{-1}}$ defined for $\varphi$ plays the role of $\boldsymbol{\gamma}_{1}$ for $\hat{t} \varphi$. Hence, the statement follows from (i) and Definition 1.

We record the following consequence of the above proof.

COROLlary 3.10. Let $\mathfrak{l}^{*}(\alpha) \geqslant 2 j-n$ for $\alpha \in \operatorname{Irr}\left(\mathrm{GL}_{j}^{\epsilon}(q)\right)$ and express $\alpha$ as in (3.10). If $\Theta$ denotes the bijection in Theorem 3.9(i), then $\Theta(\varphi)=\alpha$, where $\varphi$ is as defined in (3.9), with $\boldsymbol{\lambda}=\boldsymbol{\gamma}_{1}$ obtained by adding $n-j$ to the front of $\boldsymbol{v}=\left(\lambda_{2}, \ldots, \lambda_{r}\right): \lambda=\left(n-j, \lambda_{2}, \ldots, \lambda_{r}\right)$.

EXAMPLE 3.11.

(i) It is well known (see, for example, [T1]) that $\tau_{n}$ is the sum of $1_{G}$ and some irreducible Weil characters of $G=\mathrm{GL}_{n}(q)$, and every Weil character, 
multiplied by a suitable linear character, occurs in $\tau_{n}$. Thus, $1_{G}$ has level 0 , and Weil characters are precisely the characters of level 1.

(ii) The Steinberg character of $\mathrm{GL}_{n}(q)$ is the unipotent character corresponding to the partition $\left(1^{n}\right)$; hence, it has level $n-1$.

(iii) Consider the case of $G=\mathrm{GL}_{2}(q)$ and its irreducible characters, as labeled in [DM2, Table 1]. The $q-1$ characters of degree 1 have level 0 . The $q-1$ characters of degree $q$ (including the Steinberg character) and the $(q-1)(q-2) / 2$ characters of degree $q+1$ are all of level 1 . Finally, the remaining $q(q-1) / 2$ characters of degree $q-1$ are all of level 2 .

\section{EXAMPLE 3.12.}

(i) It is well known (see, for example, [TZ]) that $\zeta_{n}$ is a sum of irreducible Weil characters of $G=\mathrm{GU}_{n}(q)$, and every Weil character, multiplied by a suitable linear character, occurs in $\zeta_{n}$. Thus, Weil characters of $G$ are precisely the characters of level 1 .

(ii) The Steinberg character of $\mathrm{GU}_{n}(q)$ is the unipotent character corresponding to the partition $\left(1^{n}\right)$, hence it has level $n-1$.

In the case $\epsilon=+$, that is, $G=\mathrm{GL}(V) \cong \mathrm{GL}_{n}(q)$, sometimes it is convenient for us to use the Dipper-James classification of complex irreducible characters of $G$, as described in [J1]. Namely, suppose $\chi \in \operatorname{Irr}(G)$ is labeled by $s \in G \cong G^{*}$ and $\psi$ as in (3.9). The Frobenius map $F: x \mapsto x^{q}$ acts on the set of eigenvalues of $s$ acting on $V \otimes_{\mathbb{F}_{q}} \overline{\mathbb{F}}_{q}$; let $s_{1}, s_{2}, \ldots, s_{m}$ be a full set of representatives of $F$ orbits on it. Then one has

$$
\mathbf{C}_{G}(s) \cong \mathrm{GL}_{k_{1}}\left(q^{d_{1}}\right) \times \mathrm{GL}_{k_{2}}\left(q^{d_{2}}\right) \times \cdots \times \mathrm{GL}_{k_{m}}\left(q^{d_{m}}\right),
$$

where $k_{i} \mathbb{Z}_{\geqslant 1}, s_{i}$ has degree $d_{i}=\left[\mathbb{F}_{q}\left(s_{i}\right): \mathbb{F}_{q}\right]$, and $\sum_{i=1}^{m} k_{i} d_{i}=n$. Correspondingly, the unipotent character $\psi$ of $\mathbf{C}_{G}(s)$ can be written as

$$
\psi=\psi^{\lambda_{1}} \otimes \psi^{\lambda_{2}} \otimes \cdots \otimes \psi^{\lambda_{m}},
$$

where $\psi^{\lambda_{i}}$ is the unipotent character of $\mathrm{GL}_{k_{i}}\left(q^{d_{i}}\right)$ labeled by the partition $\lambda_{i} \vdash k_{i}$. Then the Dipper-James label of $\chi$ is

$$
\chi=S\left(s_{1}, \lambda_{1}\right) \circ S\left(s_{2}, \lambda_{2}\right) \circ \cdots \circ S\left(s_{m}, \lambda_{m}\right) .
$$

In fact, in (3.12), we will use the convention that if $1 \in\left\{s_{1}, \ldots, s_{m}\right\}$, then $s_{1}=1$, and if $1 \notin\left\{s_{1}, \ldots, s_{m}\right\}$, then we artificially add $S\left(s_{1}, \lambda_{1}\right)$ with $\lambda_{1}=(0)$. With this convention, we have $\boldsymbol{\gamma}_{1}=\lambda_{1}$ and can reformulate part of Theorem 3.9 for $\mathrm{GL}_{n}(q)$ as follows. 
THEOREM 3.13. Let $\chi$ be any irreducible character of $G_{n}=\operatorname{GL}_{n}(q)$, written as in (3.12). Let $0 \leqslant j \leqslant n$ be an integer.

(i) Then $\chi$ has true level $j$ precisely when the first part of the partition $\lambda_{1}$ is $n-j$.

(ii) Suppose $\mathfrak{l}^{*}(\chi)=n-j$. Let $\bar{\lambda}_{1}$ denote the partition obtained from $\lambda_{1}$ by removing the first part $n-j$, and let

$$
\alpha=S\left(s_{1}, \bar{\lambda}_{1}\right) \circ S\left(s_{2}, \lambda_{2}\right) \circ \cdots \circ S\left(s_{m}, \lambda_{m}\right) .
$$

Then $\alpha \in \operatorname{Irr}\left(G_{j}\right), \mathfrak{l}^{*}(\alpha) \geqslant 2 j-n$, and $\alpha=\left({ }^{*} R_{G_{j} \times G_{n-j}}^{G_{n}}(\varphi)\right)^{1_{G_{n-j}}}$. Furthermore, the map $\Theta: \chi \mapsto \alpha$ yields a bijection between

$$
\left\{\theta \in \operatorname{Irr}\left(\mathrm{GL}_{n}(q)\right) \mid \mathfrak{l}^{*}(\theta)=j\right\}
$$

and

$$
\left\{v \in \operatorname{Irr}\left(\mathrm{GL}_{j}(q)\right) \mid \mathfrak{l}^{*}(v) \geqslant 2 j-n\right\} .
$$

(iii) $\chi$ has level $j$ precisely when the longest among the first parts of the partitions $\lambda_{i}$ with $s_{i} \in \mathbb{F}_{q}^{\times}$is $n-j$.

\section{Further results on character levels}

Recall that if $\mathcal{G}$ is a connected reductive algebraic group and $F: \mathcal{G} \rightarrow \mathcal{G}$ is a Frobenius endomorphism, then the Alvis-Curtis (duality) isometry $D_{\mathcal{G}}$ sends any irreducible character of $G:=\mathcal{G}^{F}$ to an irreducible character, up to a sign, see [DM2, Ch. 8], and defines an involutive unitary transformation on the space $\mathcal{C}(G)$ of complex-valued class functions on $G$. If $\left(\mathcal{G}^{*}, F^{*}\right)$ is dual to $(\mathcal{G}, F)$ and $G^{*}:=\left(\mathcal{G}^{*}\right)^{F^{*}}$, then $\mathcal{E}(G,(s))$ denotes the rational series corresponding to the $G^{*}$-conjugacy class of a semisimple element $s \in G^{*}$; see [DM2, page 136]. Furthermore, let $\varepsilon_{\mathcal{G}}:=(-1)^{\sigma_{\mathcal{G}}}$, where $\sigma_{\mathcal{G}}$ is the relative rank of $\mathcal{G}$, as defined on [C, page 197].

LEMMA 4.1. In the above notation, the following statements hold.

(i) The Alvis-Curtis isometry respects rational series of irreducible characters of $G$.

(ii) Suppose $f \in \mathcal{C}(G)$ is such that $f(g)=f(s)$ whenever $s$ is the semisimple part of $g \in G$. Then $D_{\mathcal{G}}(f \alpha)=f D_{\mathcal{G}}(\alpha)$ for any $\alpha \in \mathcal{C}(G)$. 
Proof. (i) If $\mathcal{T}$ is any $F$-stable maximal torus, then note by [DM2, Definition 8.8] that $D_{\mathcal{T}}$ acts trivially on $\mathcal{C}\left(\mathcal{T}^{F}\right)$. Hence, by [DM2, Proposition 8.11], for any character $\theta \in \operatorname{Irr}\left(\mathcal{T}^{F}\right)$, we have that

$$
D_{\mathcal{G}}\left(R_{\mathcal{T}}^{\mathcal{G}}(\theta)\right)=\varepsilon_{\mathcal{G}} \varepsilon_{\mathcal{T}} R_{\mathcal{T}}^{\mathcal{G}}\left(D_{\mathcal{T}}(\theta)\right)=\varepsilon_{\mathcal{G}} \varepsilon_{\mathcal{T}} R_{\mathcal{T}}^{\mathcal{G}}(\theta)= \pm R_{\mathcal{T}}^{\mathcal{G}}(\theta),
$$

whence the statement follows.

(ii) Let $\mathcal{L}$ be an $F$-stable Levi subgroup of an $F$-stable parabolic subgroup of $\mathcal{G}$, and let ${ }^{*} R_{\mathcal{L}}^{\mathcal{G}}$ denote the Harish-Chandra restriction. By [DM2, Proposition 12.6], we have

$$
\left.R_{\mathcal{L}}^{\mathcal{G}}\left({ }^{*} R_{\mathcal{L}}^{\mathcal{G}}(f \alpha)\right)=R_{\mathcal{L}}^{\mathcal{G}}\left(\left.\left({ }^{*} R_{\mathcal{L}^{\mathcal{G}}}^{\alpha}\right) \cdot f\right|_{\mathcal{L}^{F}}\right)=f \cdot R_{\mathcal{L}}^{\mathcal{G}}{ }^{*} R_{\mathcal{L}}^{\mathcal{G}}(\alpha)\right) .
$$

Now the statement follows by applying [DM2, Definition 8.8].

Let $\mathcal{G}=\mathrm{GL}_{n}\left(\overline{\mathbb{F}}_{p}\right)$ and let $F$ denote the Frobenius endomorphism $X=$ $\left(x_{i j}\right) \mapsto X^{(q)}:=\left(x_{i j}^{q}\right)$ or $F: X \mapsto\left({ }^{t} X^{(q)}\right)^{-1}$ so that $\mathcal{G}^{F} \cong \mathrm{GL}_{n}(q)$, respectively $\mathrm{GU}_{n}(q)$. Following [FS, Section 1], we will always fix an $F$-stable maximal torus $\mathcal{T}_{1}$ consisting of diagonal matrices so that $\left|\mathcal{T}_{1}{ }^{F}\right|=(q-1)^{n}$, respectively $(q+1)^{n}$. Then the $\mathcal{G}^{F}$-conjugacy classes of maximal tori in $\mathcal{G}$ are parametrized by conjugacy classes in the Weyl group $W=\mathbf{N}_{\mathcal{G}}\left(\mathcal{T}_{1}\right) / \mathcal{T}_{1} \cong \mathrm{S}_{n}$. Furthermore, the unipotent characters of $\mathcal{G}^{F}$ are parametrized by the irreducible characters $\lambda$ of $W$, which, in turn, are parametrized by partitions $\lambda \vdash n$. For $w \in W$, let $\mathcal{T}_{w}$ denote an $F$-stable maximal torus of $\mathcal{G}$ corresponding to the $W$-conjugacy class of $w$. Then, for any $\lambda \in \operatorname{Irr}(W)$ labeled by $\lambda \vdash n$, the corresponding unipotent character $\psi^{\lambda}=\psi^{\lambda}$ of $\mathcal{G}^{F}$ is given by

$$
\psi^{\lambda}=\frac{a_{\lambda}}{|W|} \sum_{w \in W} \lambda(w) R_{\mathcal{T}_{w}^{\mathcal{G}}}\left(1_{\mathcal{T}_{w}^{F}}\right)
$$

for some $a_{\lambda}= \pm 1$; see [FS, (1.13)]. The same construction extends to direct products of groups of type GL, equipped with Frobenius endomorphisms stabilizing each factor, in particular, to $F$-stable Levi subgroups of $\mathrm{GL}_{n}$.

LEMMA 4.2. Let $\mathcal{G}=\mathcal{G}_{1} \times \mathcal{G}_{2} \times \cdots \times \mathcal{G}_{m}$ be a direct product of algebraic groups $\mathcal{G}_{i}=\mathrm{GL}_{n_{i}}$ and let $F: \mathcal{G} \rightarrow \mathcal{G}$ be a Frobenius endomorphism that stabilizes each factor $\mathcal{G}_{i}$. Let

$$
W=W_{1} \times W_{2} \times \cdots \times W_{m} \cong \mathrm{S}_{n_{1}} \times \mathrm{S}_{n_{2}} \times \cdots \times \mathrm{S}_{n_{m}}
$$

be the Weyl group of $\mathcal{G}$. For any $\lambda \in \operatorname{Irr}(W)$, let $\psi^{\lambda}$ denote the corresponding unipotent character of $\mathcal{G}^{F}$. Then

$$
D_{\mathcal{G}}\left(\psi^{\lambda}\right)= \pm \psi^{\lambda \cdot \mathbf{s g n}}
$$


where $\mathbf{s g n} \in \operatorname{Irr}(W)$ is the character that sends each permutation $\pi \in \mathrm{S}_{n_{i}}$ to the sign of $\pi$.

Proof. We apply (4.2) to $\psi^{\lambda}$. Note that $\varepsilon_{\mathcal{G}} \mathcal{E} \mathcal{T}_{w}=(-1)^{l(w)}$ by [DM2, Exercise 13.29], where $l(w)$ is the length of the element $w$ in $W$. Since $W_{i}$ is of type $A$, we have $(-1)^{l(w)}=\operatorname{sgn}(w)$. Hence, (4.1) implies that

$$
D_{\mathcal{G}}\left(R_{\mathcal{T}_{w}^{\mathcal{G}}}^{\mathcal{G}}\left(1_{\mathcal{T}_{w}^{F}}\right)\right)=\varepsilon_{\mathcal{G}} \varepsilon_{\mathcal{T}_{w}} R_{\mathcal{T}_{w}}^{\mathcal{G}}\left(1_{\mathcal{T}_{w}^{F}}\right)=\operatorname{sgn}(w) R_{\mathcal{T}_{w}^{\mathcal{G}}}\left(1_{\mathcal{T}_{w}^{F}}\right) .
$$

Applying $D_{\mathcal{G}}$ to (4.2), we obtain that $D_{\mathcal{G}}\left(\psi^{\lambda}\right)=a_{\lambda} a_{\lambda \cdot \mathbf{s g n}} \psi^{\lambda \cdot \mathbf{s g n}}$.

Now we return to $\mathcal{G}=\mathrm{GL}_{n}$ with the aforementioned Frobenius endomorphism $F: \mathcal{G} \rightarrow \mathcal{G}$ so that $G:=\mathcal{G}^{F}=\mathrm{GL}_{n}(q)$ or $\mathrm{GU}_{n}(q)$. As before, we can identify the dual group $G^{*}$ with $G$. For any semisimple element $s \in G$,

$$
\mathcal{L}:=\mathbf{C}_{\mathcal{G}}(s)=\mathcal{G}_{1} \times \mathcal{G}_{2} \times \cdots \times \mathcal{G}_{m}
$$

is of the form described in Lemma 4.2, and likewise the Weyl group

$$
W_{\mathcal{L}} \cong \mathrm{S}_{n_{1}} \times \mathrm{S}_{n_{2}} \times \cdots \times \mathrm{S}_{n_{m}}
$$

of $\mathcal{L}$ is a direct product of symmetric groups. Hence, any unipotent character $\psi^{\mu}$ of $\mathbf{C}_{G}(s)$ is labeled by an irreducible character $\mu \in \operatorname{Irr}\left(W_{\mathcal{L}}\right)$, as described in (4.2). We will keep the notation sgn as in Lemma 4.2.

PROPOSITION 4.3. In the above-introduced notation, let $\chi^{s, \mu}$ denote the irreducible character of $G=\mathcal{G}^{F}$ labeled by a semisimple element $s \in G$ and the unipotent character $\psi^{\mu} \in \operatorname{Irr}\left(\mathbf{C}_{G}(s)\right)$ corresponding to $\mu \in \operatorname{Irr}\left(W_{\mathcal{L}}\right)$, as in [FS, (1.18)]. Then

$$
D_{\mathcal{G}}\left(\chi^{s, \mu}\right)= \pm \chi^{s, \mu \cdot \mathbf{s g n}} .
$$

Proof. For $w \in W_{\mathcal{L}}$, let $\mathcal{T}_{w}$ denote an $F$-stable maximal torus of $\mathcal{L}=\mathbf{C}_{\mathcal{G}}(s)$ corresponding to the $W_{\mathcal{L}}$-conjugacy class of $w$. Then, according to [FS, (1.18)], we have that

$$
\chi^{s, \mu}=\frac{a_{s, \mu}}{\left|W_{\mathcal{L}}\right|} \sum_{w \in W_{\mathcal{L}}} \mu(w) R_{\mathcal{T}_{w}}^{\mathcal{G}}\left(\left.\hat{s}\right|_{\mathcal{T}_{w}^{F}}\right)
$$

for some $a_{s, \mu}= \pm 1$, where the linear character $\hat{s}$ of $\mathbf{C}_{G}(s)$ is introduced before (3.9). As mentioned in the proof of Lemma $4.2, \varepsilon_{\mathcal{T}_{w}}=\operatorname{sgn}(w) \varepsilon_{\mathcal{L}}$. Applying (4.1), we obtain that

$$
D_{\mathcal{G}}\left(R_{\mathcal{T}_{w}}^{\mathcal{G}}\left(\left.\hat{s}\right|_{\mathcal{T}_{w}^{F}}\right)\right)=\varepsilon_{\mathcal{G}} \mathcal{E}_{\mathcal{T}_{w}} R_{\mathcal{T}_{w}}^{\mathcal{G}}\left(\left.\hat{s}\right|_{\mathcal{T}_{w}^{F}}\right)=\operatorname{sgn}(w) \varepsilon_{\mathcal{G}} \varepsilon_{\mathcal{L}} R_{\mathcal{T}_{w}^{\mathcal{G}}}\left(\left.\hat{s}\right|_{\mathcal{T}_{w}^{F}}\right) .
$$

Applying $D_{\mathcal{G}}$ to (4.3), we then arrive at

$$
D_{\mathcal{G}}\left(\chi^{s, \mu}\right)=a_{s, \mu} a_{s, \mu \cdot \mathbf{s g n}} \varepsilon_{\mathcal{G}} \varepsilon_{\mathcal{L}} \chi^{s, \mu \cdot \mathbf{s g n}} .
$$


Now we can link the levels of $\chi \in \operatorname{Irr}(G)$ and its Alvis-Curtis dual. Slightly abusing the notation, we define $\mathfrak{l}(-\chi):=\mathfrak{l}(\chi)$ for any $\chi \in \operatorname{Irr}(G)$.

PROPOSITION 4.4. Let $\mathcal{G}=\mathrm{GL}_{n}$ and $G=\mathcal{G}^{F}=\mathrm{GL}_{n}(q)$ or $\mathrm{GU}_{n}(q)$ for $a$ Frobenius endomorphism $F: \mathcal{G} \rightarrow \mathcal{G}$. Then for any $\chi \in \operatorname{Irr}(G)$,

$$
\mathfrak{l}(\chi)+\mathfrak{l}\left(D_{\mathcal{G}}(\chi)\right) \geqslant n-1 .
$$

Proof. The proofs for $\mathrm{GL}_{n}(q)$ and $\mathrm{GU}_{n}(q)$ are identical, so we will give the details in the case $G=\mathrm{GU}_{n}(q)$. Let $\mathfrak{l}(\chi)=j$ and apply Theorem 3.9 to $\chi$. Then the longest part among all the parts of the partitions $\gamma_{\delta}, \delta \in \mu_{q-\epsilon 1}$, is precisely $n-j$ if we express $\chi$ as in (3.8) and (3.9). Say the partition $\boldsymbol{\gamma}_{\epsilon_{0}}$ has the longest part $k_{\epsilon_{0}}=n-j$.

According to Proposition $4.3, D_{\mathcal{G}}(\chi)= \pm \chi^{s, \mu \cdot \mathbf{s g n}}$, and so the unipotent character of $\mathbf{C}_{G}(s)$ corresponding to $D_{\mathcal{G}}(\chi)$ has $\epsilon$-component equal to $\psi^{\gamma_{\delta}}$. $\operatorname{sgn}=\psi^{\boldsymbol{\gamma}_{\delta}^{\prime}}$, where $\boldsymbol{\gamma}_{\delta}^{\prime}$ is the partition conjugate to $\boldsymbol{\gamma}_{\delta} \vdash n_{\delta}$, with the longest part $k_{\delta}^{\prime}$. Now, if $\epsilon \neq \epsilon_{0}$, then

$$
k_{\delta}^{\prime} \leqslant n_{\delta} \leqslant n-n_{\epsilon_{0}} \leqslant n-k_{\epsilon_{0}}=j .
$$

Furthermore, since $\boldsymbol{\gamma}_{\epsilon_{0}}$ and $\boldsymbol{\gamma}_{\epsilon_{0}}^{\prime}$ are conjugate partitions, we have

$$
k_{\epsilon_{0}}^{\prime} \leqslant n_{\epsilon_{0}}+1-k_{\epsilon_{0}} \leqslant n+1-k_{\epsilon_{0}}=j+1 .
$$

We have shown that $\max \left\{k_{\delta}^{\prime} \mid \delta \in \mu_{q-\epsilon 1}\right\} \leqslant j+1$. Hence, $\mathfrak{l}\left(D_{\mathcal{G}}(\chi)\right) \geqslant n-j-1$ by Theorem 3.9.

The example of $\chi=1_{G}$ shows that the bound in Proposition 4.4 is sharp since $\pm D_{\mathcal{G}}\left(1_{G}\right)$ is the Steinberg character of $G$.

Next, for a fixed $\epsilon= \pm$, we consider $G_{n}=\operatorname{GL}_{n}^{\epsilon}(q)=\mathcal{G}^{F}, G_{k}=\mathrm{GL}_{k}^{\epsilon}(q)=$ $\left(\mathcal{G}_{k}\right)^{F}$ with $\mathcal{G}_{k}=\mathrm{GL}_{k}$ for $1 \leqslant k \leqslant n$, and define $\omega_{n}:=\tau_{n}$ if $\epsilon=+$ and $\omega_{n}:=$ $(-1)^{n} \zeta_{n}$ if $\epsilon=-$. In what follows, the notation $(\mathcal{T}) \subset \mathcal{G}_{k}$ means that we sum over a set of representatives of $G_{k}$-conjugacy classes of $F$-stable maximal tori $\mathcal{T}$ in $\mathcal{G}_{k}$; furthermore, we set $T:=\mathcal{T}^{F}$ and $W(T):=\mathbf{N}_{G_{k}}(\mathcal{T}) / T$ for any such $\mathcal{T}$.

We will need the following identity.

LEMMA 4.5. For any $m \in \mathbb{Z}_{\geqslant 0}$, we have

$$
t^{m}=\sum_{k=0}^{m}\left(\begin{array}{l}
m \\
k
\end{array}\right) \prod_{q}^{k-1}\left(t-q^{i}\right) .
$$


Proof. We proceed by induction on $m$, with trivial base case $m=0$. For the induction step $m \geqslant 2$, note that if $1 \leqslant k \leqslant m-1$, then

$$
\left(\begin{array}{l}
m \\
k
\end{array}\right)_{q}=\left(\begin{array}{c}
m-1 \\
k
\end{array}\right)_{q}+q^{m-k}\left(\begin{array}{c}
m-1 \\
k-1
\end{array}\right)_{q} .
$$

Applying the induction hypothesis to the two indeterminates $t^{\prime}=t / q$ and $q$, we have

$$
\begin{aligned}
\sum_{k=1}^{m} q^{m-k}\left(\begin{array}{c}
m-1 \\
k-1
\end{array}\right) \prod_{q}^{k-1}\left(t-q^{i}\right) & =(t-1) q^{m-1} \sum_{k=1}^{m}\left(\begin{array}{c}
m-1 \\
k-1
\end{array}\right) \prod_{q}^{k-2}\left(t^{\prime}-q^{i}\right) \\
& =(t-1) q^{m-1} \sum_{j=0}^{m-1}\left(\begin{array}{c}
m-1 \\
j
\end{array}\right) \prod_{q}^{j-1}\left(t^{\prime}-q^{i}\right) \\
& =(t-1) q^{m-1} t^{\prime m-1}=(t-1) t^{m-1} .
\end{aligned}
$$

Combined with (4.4), it yields that

$$
\begin{aligned}
\sum_{k=0}^{m}\left(\begin{array}{c}
m \\
k
\end{array}\right) \prod_{q}^{k-1}\left(t-q^{i}\right)= & \sum_{k=0}^{m-1}\left(\begin{array}{c}
m-1 \\
k
\end{array}\right) \prod_{q}^{k-1}\left(t-q^{i}\right) \\
& +\sum_{k=0}^{m} q^{m-k}\left(\begin{array}{c}
m-1 \\
k-1
\end{array}\right) \prod_{q}\left(t-q^{i}\right) \\
= & t^{m-1}+(t-1) t^{m-1}=t^{m} .
\end{aligned}
$$

Now we can prove the following result, which gives a decomposition for $\left(\omega_{n}\right)^{m}$ in terms of Deligne-Lusztig characters. In the case $q$ is sufficiently large, this statement follows from the main result of [S2].

TheOREM 4.6. Fix $\epsilon= \pm$ and let $G_{k}=\mathrm{GL}_{k}^{\epsilon}(q)$ for $1 \leqslant k \leqslant n$. Then, for any $1 \leqslant m \leqslant n$,

$$
\begin{aligned}
\left(\omega_{n}\right)^{m} & =\sum_{k=0}^{m} \sum_{(\mathcal{T}) \subset \mathcal{G}_{k}} \frac{\epsilon^{k}}{|W(T)|} \sum_{\theta \in \operatorname{Irr}(T)} R_{T \times G_{m-k}}^{G_{m}}\left(\theta \otimes 1_{G_{m-k}}\right)(1) R_{T \times G_{n-k}}^{G_{n}}\left(\theta \otimes 1_{G_{n-k}}\right) \\
& =\sum_{k=0}^{m} \sum_{(\mathcal{T}) \subset \mathcal{G}_{k}} \frac{\epsilon^{k} \varepsilon_{\mathcal{G}_{m}} \varepsilon_{\mathcal{G}_{m-k}} \varepsilon_{\mathcal{T}}}{|W(T)|} \cdot \frac{\left|G_{m}\right|_{p^{\prime}}}{|T| \cdot\left|G_{m-k}\right|_{p^{\prime}}} \sum_{\theta \in \operatorname{Irr}(T)} R_{T \times G_{n-k}}^{G_{n}}\left(\theta \otimes 1_{G_{n-k}}\right) .
\end{aligned}
$$

Proof. First, we note that the second equality in the statement follows from the degree formula for $R_{T \times G_{m-k}}^{G_{m}}\left(\theta \otimes 1_{G_{m-k}}\right)$ (see, for example, [S2, (2.3) and (2.6)]). 
Next, we can replace the summation $\sum_{(\mathcal{T}) \subset \mathcal{G}_{k}} \frac{1}{|W(T)| \cdot|T|}$ by $\frac{1}{\left|G_{k}\right|} \sum_{\mathcal{T} \subset \mathcal{G}_{k}}$, where the second summation runs over all $F$-stable maximal tori $\mathcal{T}$ in $\mathcal{G}_{k}$. Since $\varepsilon_{\mathcal{G}_{n}}=$ $\epsilon^{n(n-1) / 2}$, we also see that

$$
\varepsilon_{\mathcal{G}_{m}} \varepsilon_{\mathcal{G}_{m-k}}=\varepsilon_{\mathcal{G}_{k}} \epsilon^{k m-k}
$$

for $0 \leqslant k \leqslant m$. Hence, the right-hand side $R$ of the formula in Theorem 4.6 is

$$
\begin{aligned}
& R=\sum_{k=0}^{m} \sum_{\mathcal{T} \subset \mathcal{G}_{k}} \frac{\varepsilon_{\mathcal{G}_{k}} \mathcal{T}_{\mathcal{T}} \epsilon^{k m}\left|G_{m}\right|_{p^{\prime}}}{\left|G_{m-k}\right|_{p^{\prime}} \cdot\left|G_{k}\right|} \sum_{\theta \in \operatorname{Irr}(T)} R_{T \times G_{n-k}}^{G_{n}}\left(\theta \otimes 1_{G_{n-k}}\right) \\
&=\sum_{k=0}^{m} \frac{\epsilon^{k m}\left|G_{m}\right|_{p^{\prime}}}{\left|G_{m-k}\right|_{p^{\prime}} \cdot\left|G_{k}\right|} \sum_{\mathcal{T} \subset \mathcal{G}_{k}, \theta \in \operatorname{Irr}(T)} \varepsilon_{\mathcal{G}_{k}} \varepsilon_{\mathcal{T}} R_{T \times G_{n-k}}^{G_{n}}\left(\theta \otimes 1_{G_{n-k}}\right) \\
& \stackrel{\text { Corollary } 2.6}{=} \sum_{k=0}^{m} \frac{\epsilon^{k m}\left|G_{m}\right|_{p^{\prime}}}{\left|G_{m-k}\right|_{p^{\prime}} \cdot\left|G_{k}\right|_{p^{\prime}}} R_{G_{k} \times G_{n-k}}^{G_{n}}\left(\sum_{\mathcal{T} \subset \mathcal{G}_{k}, \theta \in \operatorname{Irr}(T)} \frac{\varepsilon_{\mathcal{G}_{k}} \varepsilon_{\mathcal{T}}}{\left|G_{k}\right|_{p}} R_{T}^{G_{k}}(\theta) \otimes 1_{G_{n-k}}\right) \\
& \stackrel{\text { Propositions } 3.2,3.6}{=} \sum_{k=0}^{m} \frac{\epsilon^{k(m-k)}\left|G_{m}\right|_{p^{\prime}}}{\left|G_{m-k}\right|_{p^{\prime}} \cdot\left|G_{k}\right|_{p^{\prime}}} \prod_{i=0}^{k-1}\left(\omega_{n}-(\epsilon q)^{i} \cdot 1_{G_{n}}\right) \\
&=\sum_{k=0}^{m}\left(\begin{array}{c}
m \\
k
\end{array}\right)_{\epsilon q} \prod_{i=0}^{k-1}\left(\omega_{n}-(\epsilon q)^{i} \cdot 1_{G_{n}}\right) \stackrel{\epsilon^{k m}\left|G_{m}\right|_{p^{\prime}}}{\left|G_{m-k}\right|_{p^{\prime}} \cdot\left|G_{k}\right|_{p^{\prime}}} R_{G_{k} \times G_{n-k}}^{G_{n}}\left(\operatorname{reg}_{G_{k}} \otimes 1_{G_{n-k}}\right) \\
&=
\end{aligned}
$$

(Here, at the last step, we apply Lemma 4.5 to $t=\omega_{n}(g)$ and $z=\epsilon q$ for every $g \in G_{n}$.)

In the next result, which is a GU-analogue of (2.2), the first equality was known in the case $q$ is sufficiently large; cf. [S2].

COROLlaRY 4.7. Let $q$ be a power of a prime $p, G_{k}=\mathrm{GU}_{k}(q)$ for $1 \leqslant k \leqslant n$. Then for any $1 \leqslant m \leqslant n$,

$$
\begin{aligned}
{\left[\left(\zeta_{n}\right)^{m}, 1_{G_{n}}\right]_{G_{n}} } & =\sum_{k=0}^{m} \frac{(-1)^{(k+n) m}\left|G_{m}\right|_{p^{\prime}}}{\left|G_{m-k}\right|_{p^{\prime}} \cdot\left|G_{k}\right|_{p^{\prime}}} \\
& = \begin{cases}0, & 2 \nmid m, \\
(q+1)\left(q^{3}+1\right) \cdots\left(q^{m-1}+1\right), & 2 \mid m .\end{cases}
\end{aligned}
$$

In particular, if $1 \leqslant j \leqslant n / 2$ and $V=\mathbb{F}_{q^{2}}^{n}$ is the natural $\mathrm{GU}_{n}(q)$-module, then the number of $\mathrm{GU}_{n}(q)$-orbits on ordered $j$-tuples $\left(v_{1}, \ldots, v_{j}\right)$ with $v_{i} \in V$ is $\prod_{i=1}^{j}\left(q^{2 i-1}+1\right)$. 
Proof. It follows from the proof of Theorem 4.6 that

$$
\left(\zeta_{n}\right)^{m}=(-1)^{m n}\left(\omega_{n}\right)^{m}=\sum_{k=0}^{m} \frac{\epsilon^{(k+n) m}\left|G_{m}\right|_{p^{\prime}}}{\left|G_{m-k}\right|_{p^{\prime}} \cdot\left|G_{k}\right|_{p^{\prime}}} R_{G_{k} \times G_{n-k}}^{G_{n}}\left(\operatorname{reg}_{G_{k}} \otimes 1_{G_{n-k}}\right) .
$$

Taking the inner product and using [DM2, Corollary 12.7], we get

$$
\begin{aligned}
{\left[\left(\zeta_{n}\right)^{m}, 1_{G_{n}}\right]_{G_{n}} } & =\sum_{k=0}^{m} \frac{\epsilon^{(k+n) m}\left|G_{m}\right|_{p^{\prime}}}{\left|G_{m-k}\right|_{p^{\prime}} \cdot\left|G_{k}\right|_{p^{\prime}}}\left[\mathbf{r e g}_{G_{k}} \otimes 1_{G_{n-k}},{ }^{*} R_{G_{k} \times G_{n-k}}^{G_{n}}\left(1_{G_{n}}\right)\right]_{G_{k} \times G_{n-k}} \\
& =\sum_{k=0}^{m} \frac{\epsilon^{(k+n) m}\left|G_{m}\right|_{p^{\prime}}}{\left|G_{m-k}\right|_{p^{\prime}} \cdot\left|G_{k}\right|_{p^{\prime}}}\left[\operatorname{reg}_{G_{k}} \otimes 1_{G_{n-k}}, 1_{G_{k} \times G_{n-k}}\right]_{G_{k} \times G_{n-k}} \\
& =\sum_{k=0}^{m} \frac{\epsilon^{(k+n) m}\left|G_{m}\right|_{p^{\prime}}}{\left|G_{m-k}\right|_{p^{\prime}} \cdot\left|G_{k}\right|_{p^{\prime}}} .
\end{aligned}
$$

When $2 \nmid m$, the terms for $k$ and $m-k, 0 \leqslant k<m / 2$, in the last summation cancel each other, yielding $\left[\left(\zeta_{n}\right)^{m}, 1_{G_{n}}\right]_{G_{n}}=0$. If $2 \mid m$, the last summation is $\sum_{k=0}^{m}(-1)^{k}\left(\begin{array}{l}m \\ k\end{array}\right)_{-q}$, and so it is $(q+1)\left(q^{3}+1\right) \cdots\left(q^{m-1}+1\right)$ by Gauss' formula (see [Ku, (1.7b)]).

Finally, the last statement follows by applying the formula we just proved to $m=2 j$.

Corollary 4.7 implies the following parity phenomenon for unitary groups.

COROllary 4.8. Suppose that $0 \leqslant i, j \leqslant i+j \leqslant n$, and $2 \nmid(i+j)$. Then the $\mathrm{GU}_{n}(q)$-characters $\left(\zeta_{n}\right)^{i}$ and $\left(\zeta_{n}\right)^{j}$ have no common irreducible constituents. In particular, if $0 \leqslant j \leqslant n / 2$, then $\left(\zeta_{n}\right)^{j}$ contains only irreducible characters of true level $j-2 t, 0 \leqslant t \leqslant j / 2$.

Proof. Note that $\left[\left(\zeta_{n}\right)^{i},\left(\zeta_{n}\right)^{j}\right]_{G_{n}}=\left[\left(\zeta_{n}\right)^{i+j}, 1_{G_{n}}\right]_{G_{n}}$. Hence, the statements follow from Corollary 4.7 and Definition 1.

\section{Bounds on character degrees}

Recall that $q=p^{f} \geqslant 2$ and $\epsilon= \pm$, we let $\mathrm{GL}_{n}^{\epsilon}(q)$ denote $\mathrm{GL}_{n}(q)$ if $\epsilon=+$ and $\mathrm{GU}_{n}(q)$ if $\epsilon=-$. In this section, we prove Theorem 1.2. For values of $j$ small compared to $n$, this gives rather tight bounds on the degree of characters $\chi$ of $\mathrm{GL}_{n}^{\epsilon}(q)$ of level $j$. The idea behind the proof is that $\chi$ is obtained by Lusztig induction from a character of the form $\alpha \otimes \beta$ of $\mathrm{GL}_{a}^{\epsilon}(q) \times \mathrm{GL}_{b}^{\epsilon}(q)$, where $\alpha$ is a unipotent character of low level and $b$ is small compared to $n$. From this, 
the degree of $\chi$ can be expressed as a polynomial in $q$ whose degree lies in the interval $[(j(n-j), j n]$. It is shown that the leading term of this polynomial gives a fairly good approximation to the value of the polynomial, even when $q$ is small.

Recall that $\psi^{\lambda}$ denotes the unipotent character of $\mathrm{GL}_{n}^{\epsilon}(q)$ corresponding to a partition $\lambda=\left(\lambda_{1}, \lambda_{2}, \ldots, \lambda_{r}\right) \vdash n$ (with the convention $\lambda_{1} \geqslant \lambda_{2} \geqslant \cdots \geqslant \lambda_{r} \geqslant 0$ ). For such $\lambda$, define

$$
a(\lambda):=\sum_{i=1}^{r}(i-1) \lambda_{i}, \quad b(\lambda):=\frac{1}{2}\left(n^{2}-\sum_{i=1}^{r} \lambda_{i}^{2}\right)=\sum_{1 \leqslant i<j \leqslant r} \lambda_{i} \lambda_{j}
$$

(see [L, (4.4.2)] and [GP, 5.4.2], where the function $a(\lambda)$ was introduced and where it was also noted that $b(\lambda)=n(n-1) / 2-a\left(\lambda^{\prime}\right)$ for the conjugate partition $\left.\lambda^{\prime}\right)$, and let $G_{\lambda}^{\epsilon}:=\operatorname{GL}_{\lambda_{1}}^{\epsilon}(q) \times \operatorname{GL}_{\lambda_{2}}^{\epsilon}(q) \times \cdots \times \operatorname{GL}_{\lambda_{r}}^{\epsilon}(q)$ be a Levi subgroup of $G_{n}^{\epsilon}:=\mathrm{GL}_{n}^{\epsilon}(q)$.

First, we collect some elementary estimates.

Lemma 5.1. Let $q \geqslant 2$ and $a, b \in \mathbb{Z}_{\geqslant 1}$. Then the following inequalities hold:

(i) $\prod_{i=2}^{\infty}\left(1-1 / q^{i}\right)>9 / 16$ and $\prod_{i=1}^{\infty}\left(1-1 / q^{i}\right)>(9 / 16)(1-1 / q) \geqslant 9 / 32$.

(ii) If $d \in \mathbb{Z}_{\geqslant 2}$, then $\prod_{i=1, d \nmid i}^{\infty}\left(1-1 / q^{i}\right)>(9 / 16)(1-1 / q)$.

(iii) $\left(q^{2 a}-1\right)\left(q^{2 a+1}+1\right)<q^{4 a+1}$ and $\left(q^{2 a-1}+1\right)\left(q^{2 a}-1\right)>q^{4 a-1}$.

(iv) $\prod_{i=1}^{n}\left(q^{i}-(-1)^{i}\right)>q^{n(n+1) / 2}$.

(v) If $a>b$, then

$$
\frac{q^{a}+1}{q^{b}+1}<q^{a-b}<\frac{q^{a}-1}{q^{b}-1}, \quad \frac{\left(q^{a+1}-1\right)\left(q^{a}+1\right)}{\left(q^{b+1}-1\right)\left(q^{b}+1\right)}<q^{2 a-2 b} .
$$

(vi) $q^{a b} / 2 \leqslant(q-1) q^{a b-1} \leqslant\left[G_{a+b}^{-}:\left(G_{a}^{-} \times G_{b}^{-}\right)\right]_{p^{\prime}}<q^{a b}<\left[G_{a+b}^{+}:\left(G_{a}^{+} \times G_{b}^{+}\right)\right]_{p^{\prime}}$. Furthermore, $\left[G_{a+b}^{-}:\left(G_{a}^{-} \times G_{b}^{-}\right)\right]_{p^{\prime}} \geqslant(5 / 8) q^{a b}$ if $a \geqslant 2$, and $\left[G_{a+b}^{-}\right.$: $\left.\left(G_{a}^{-} \times G_{b}^{-}\right)\right]_{p^{\prime}}>(q-1) q^{a b-1}$ if $a+b \geqslant 3$.

Proof. (i) This is [LMT, Lemma 4.1(ii)]. Part (ii) follows from (i). Parts (iii) and (v) are obvious, and (iv) follows from (iii). It is easy to see that $q^{a-b}<\frac{q^{a}-1}{q^{b}-1}$ implies $q^{a b}<\left[G_{a+b}^{+}:\left(G_{a}^{+} \times G_{b}^{+}\right)\right]_{p^{\prime}}$. Next, if $2 \mid a$, then a repeated application of (v) yields

$$
X:=\left[G_{a+b}^{-}:\left(G_{a}^{-} \times G_{b}^{-}\right)\right]_{p^{\prime}}=\frac{\left(q^{a+b}-(-1)^{a+b}\right) \cdots\left(q^{a+2}-1\right)\left(q^{a+1}+1\right)}{\left(q^{b}-(-1)^{b}\right) \cdots\left(q^{2}-1\right)(q+1)}<q^{a b} .
$$


If $2 \nmid a$, then a repeated application of (iii) to the numerator and an application of (iv) to the denominator of $X$ yields

$$
X=\frac{\left(q^{a+b}-(-1)^{a+b}\right) \cdots\left(q^{a+2}+1\right)\left(q^{a+1}-1\right)}{\left(q^{b}-(-1)^{b}\right) \cdots\left(q^{2}-1\right)(q+1)}<\frac{q^{(a+b)+\cdots+(a+2)+(a+1)}}{q^{b+\cdots+2+1}}=q^{a b} .
$$

Assume now that $a \geqslant 2$. If $2 \mid a$, then a repeated application of (iii) shows that the numerator of $X$ is $>q^{N}$ for $N:=\sum_{i=a+1}^{a+b} i$. If $2 \nmid a$, then singling out the factor $q^{a+1}-1$ and then applying (iii) to the remaining product shows that the numerator of $X$ is $\geqslant q^{N-4}\left(q^{4}-1\right) \geqslant(15 / 16) q^{N}$. Singling out the factor $q+1$ and then applying (iii) to the remaining product shows that the denominator of $X$ is $\leqslant q^{M-1}(q+1) \leqslant(3 / 2) q^{M}$ for $M:=\sum_{i=1}^{b} i$. It follows that $X \geqslant(15 / 16)(2 / 3) q^{N-M}=(5 / 8) q^{a b}$, as well as

$$
X \geqslant\left(q^{4}-1\right) q^{N-M-3} /(q+1)>(q-1) q^{a b-1} .
$$

The same argument applies if $b \geqslant 2$. Finally, if $a=b=1$, then $X=q-1$.

LEMMA 5.2. Let $\lambda=\left(k=\lambda_{1} \geqslant \lambda_{2} \geqslant \cdots \geqslant \lambda_{r} \geqslant 0\right) \vdash n$ and $2 \leqslant k \leqslant n / 2$. Then

$$
[\lambda]:=n^{2}-2 \sum_{i=1}^{r} \lambda_{i}^{2} \geqslant 0 .
$$

In fact, either $[\lambda] \geqslant 2.4$ or one of the following statements holds:

(i) $n=3 k=6$ and $\lambda=(2,2,2)$.

(ii) $n=2 k+1$, and either $\lambda=(k, k, 1)$ or $\lambda \in\{(3,2,2),(2,1,1,1)\}$.

(iii) $n=2 k$, and either $\lambda \in\{(k, k),(k, k-1,1)\}$ or $\lambda \in\{(4,2,2),(3,1,1,1)\}$.

Proof. (a) Note that if $a, b \in \mathbb{Z}_{\geqslant 1}$ and $a \geqslant b$, then $\left((a+1)^{2}+(b-1)^{2}\right)-\left(a^{2}+\right.$ $\left.b^{2}\right) \geqslant 2$. In what follows, we will call any replacement of the pair $(a, b)$ among the $\lambda_{i}$ 's by $(a+1, b-1)$ a push-up, and note that any push-up decreases $[\lambda]$ by at least 4 .

First, suppose that $k \leqslant n / 3$. Write $n=3 c+d$ with $c=\lfloor n / 3\rfloor \geqslant k \geqslant 2$ and $0 \leqslant d \leqslant 2$. As long as $\lambda_{1}<c$, we can apply a push-up to some pair $\left(\lambda_{1}, \lambda_{j}\right)$ (where $\lambda_{j}>0$ but $\lambda_{j+1}$, if any, is 0 ) to increase $\lambda_{1}$ and decrease $[\lambda]$. Once $\lambda_{1}=c$, we can apply the same procedure to $\lambda_{2}$, and so on. This argument shows that $[\lambda]$ will be minimized when $\lambda=(c, c, c, d)$. In particular, if $c \geqslant 3$, then

$$
[\lambda] \geqslant(3 c+d)^{2}-2\left(3 c^{2}+d^{2}\right)=3 c^{2}+6 c d-d^{2} \geqslant 3 c^{2}+5 c d \geqslant 9 c+15 d \geqslant 3 n .
$$


Assume that $c=2$, that is $6 \leqslant n \leqslant 8$. If $n \geqslant 7$, then a push-up argument shows that $[\lambda] \geqslant[(2,2,2, d)]>2.4 n$. If $n=6$, but $\lambda \neq(2,2,2)$, then $[\lambda] \geqslant[(2,2$, 2)] $+4>2.4 n$.

(b) Now we may assume $n / 3<k \leqslant n / 2$, and write $n=2 k+l$ with $0 \leqslant l<k$. Again using push-ups, we see that

$$
[\lambda] \geqslant[(k, k, l)]=4 k l-l^{2} \geqslant 3 k l+l>2.4 n
$$

if $l \geqslant 2$. Suppose $l=1$ and $\lambda \neq(k, k, 1)$. Then $\lambda_{2} \leqslant k-1$ and we can push $\lambda_{2}$ up to $k-1$. In particular, if $k \geqslant 4$, then $[\lambda] \geqslant[(k, k-1,2)]=8 k-9>2.4 n$. If $k=3$ but $\lambda \neq(3,2,2)$, then $[\lambda] \geqslant[(3,2,2)]+4>2.4 n$. If $k=2$, then $\lambda=(2,1,1,1)$.

Assume now that $n=2 k$ but $\lambda \neq(k, k),(k, k-1,1)$. Then $\lambda_{2} \leqslant k-2$ and we can push $\lambda_{2}$ up to $k-2$. In particular, if $k \geqslant 5$, then $[\lambda] \geqslant[(k, k-2$, $2)]=8 k-16 \geqslant 2.4 n$. If $k=4$ but $\lambda \neq(4,2,2)$, then $[\lambda] \geqslant[(4,2,2)]+4>2.4 n$. Otherwise $k=3$ and $\lambda=(3,1,1,1)$.

Finally, $[\lambda] \geqslant 0$ in all the listed exceptions to the inequality $[\lambda] \geqslant 2.4 n$.

Note that the constant 2.4 cannot be improved since $[(5,3,2)]=24=2.4 \cdot 10$.

LEMMA 5.3. For $\epsilon= \pm$ and $\lambda=\left(k=\lambda_{1} \geqslant \lambda_{2} \geqslant \cdots \geqslant \lambda_{r}>0\right) \vdash n$, the following statements hold:

(i) $\psi^{\lambda}(1)$ and $\left[G_{n}^{\epsilon}: G_{\lambda}^{\epsilon}\right]_{p^{\prime}}$ are both monic polynomials in $q$ with integer coefficients and of degree $\operatorname{deg}_{q} \psi^{\lambda}(1)=\operatorname{deg}_{q}\left[G_{n}^{\epsilon}: G_{\lambda}^{\epsilon}\right]_{p^{\prime}}=b(\lambda)$.

(ii) If $\epsilon=+$, then $\psi^{\lambda}(1) \geqslant q^{b(\lambda)} \geqslant q^{k(n-k)}$.

(iii) If $\epsilon=-$, then

$$
\psi^{\lambda}(1) \geqslant \max \left\{\left(\frac{q}{q+1}\right)^{|\lambda|-1} \cdot q^{b(\lambda)}, \frac{1}{2} q^{k(n-k)}\right\} .
$$

(iv) $\left[G_{n}^{-}: G_{\lambda}^{-}\right]_{p^{\prime}}<q^{b(\lambda)}<\left[G_{n}^{+}: G_{\lambda}^{+}\right]_{p^{\prime}}$.

Proof. (a) The statements about $\psi^{\lambda}(1)$, respectively $\left[G_{n}^{\epsilon}: G_{\lambda}^{\epsilon}\right]_{p^{\prime}}$, being a monic polynomial in $q$ with integer coefficients, are well known. The degree formula for $\psi^{\lambda}$ is likewise well known (see, for example, [C, Section 13.8]), from which it follows that 


$$
\begin{aligned}
\operatorname{deg}_{q} \psi^{\lambda}(1)= & \left(\begin{array}{c}
n+1 \\
2
\end{array}\right)+\sum_{i=1}^{r}\left(\lambda_{i}+r-i\right)(r-i+1)-\sum_{i=1}^{r-1}\left(\begin{array}{l}
i \\
2
\end{array}\right) \\
& -\sum_{i=1}^{r}\left(\begin{array}{c}
\lambda_{i}+r-i+1 \\
2
\end{array}\right)=b(\lambda) .
\end{aligned}
$$

We also have

$$
\operatorname{deg}_{q}\left[G_{n}^{\epsilon}: G_{\lambda}^{\epsilon}\right]_{p^{\prime}}=n(n+1) / 2-\sum_{i=1}^{r} \lambda_{i}\left(\lambda_{i}+1\right) / 2=b(\lambda) .
$$

(b) For the remaining statements (ii)-(iv), we will use induction on the length $r$ of $\lambda$, with the base case $r=1$ being obvious. Let

$$
\boldsymbol{\mu}:=\left(\lambda_{2}, \lambda_{3}, \ldots, \lambda_{r}\right) \vdash(n-k) .
$$

By induction on $r$ and Lemma 5.1(vi), we have

$$
\left[G_{n}^{-}: G_{\lambda}^{-}\right]_{p^{\prime}}=\left[G_{n}^{-}: G_{k}^{-} \times G_{n-k}^{-}\right]_{p^{\prime}}\left[G_{n-k}^{-}: G_{\mu}^{-}\right]_{p^{\prime}}<q^{k(n-k)} q^{b(\mu)}=q^{b(\lambda)},
$$

yielding (iv).

For the remaining claims (ii) and (iii), we will use the quantized hook formula

$$
\psi^{\lambda}(1)=q^{a(\lambda)} \frac{(q-1)\left(q^{2}-\epsilon^{2}\right) \cdots\left(q^{n}-\epsilon^{n}\right)}{\prod_{h}\left(q^{l(h)}-\epsilon^{l(h)}\right)},
$$

where $h$ runs over all the hooks of the Young diagram of $\lambda$ and $l(h)$ denotes the length of the hook $h$ (see, for example, [Ol, (21)] or [Ma]).

Suppose $\epsilon=+$. Then by (i) and (5.2), we have

$$
\frac{\psi^{\lambda}(1) / q^{b(\lambda)}}{\psi^{\mu}(1) / q^{b(\mu)}}=\frac{\prod_{i=1}^{k}\left(1-1 / q^{n-i+1}\right)}{\prod_{i=1}^{k}\left(1-1 / q^{l\left(h_{i}\right)}\right)} \geqslant 1
$$

since $l\left(h_{1}\right) \leqslant n, l\left(h_{2}\right) \leqslant n-1, \ldots, l\left(h_{k}\right) \leqslant n-k+1$. As $\psi^{\mu}(1) \geqslant q^{b(\mu)}$ by the induction hypothesis, we get $\psi^{\lambda}(1) \geqslant q^{b(\lambda)}$. Since $b(\lambda)=b(\boldsymbol{\mu})+k(n-k) \geqslant$ $k(n-k)$, (ii) holds.

(c) From now on, we assume $\epsilon=-$. Then (i), (5.2), and Lemma 5.1(iv) imply that

$$
\frac{\psi^{\lambda}(1)}{q^{b(\lambda)}}>\frac{1}{\prod_{h}\left(1-(-1 / q)^{l(h)}\right)} .
$$

In particular, if at least one of the hooks $h$ has even length, then $\frac{\psi^{\lambda}(1)}{q^{b(\lambda)}}>\left(\frac{q}{q+1}\right)^{n-1}$. The same estimate holds if at least two hooks have odd length $\geqslant 3$ since $(1+$ 
$\left.1 / q^{3}\right)^{2}<1+1 / q$. The only $\lambda$ that have no hook of even length and at most one hook of odd length $\geqslant 3$ are (1) and $(2,1)$, for which (iii) also holds.

It remains to prove $\psi^{\lambda}(1) \geqslant q^{k(n-k)} / 2$. Suppose, in addition, that $k \leqslant n-2$. By Lemma 5.1(iii), we have

$$
\frac{\left(q^{n}-\epsilon\right)\left(q^{n-1}-\epsilon^{n-1}\right) \cdots\left(q^{n-k+1}-\epsilon^{n-k+1}\right)}{q^{n+(n-1)+\cdots+(n-k+1)}} \geqslant \frac{q^{4}-1}{q^{4}} \geqslant 15 / 16 .
$$

Note that $l\left(h_{1}\right)>l\left(h_{2}\right)>\cdots>l\left(h_{k}\right) \geqslant 1$. As $(1+x)^{-1}>e^{-x}$ for all $x>0$,

$$
\begin{aligned}
\frac{q^{l\left(h_{1}\right)+\cdots+l\left(h_{k}\right)}}{\left(q^{l\left(h_{1}\right)}-\epsilon^{l\left(h_{1}\right)}\right) \cdots\left(q^{\left(l\left(h_{k}\right)\right.}-\epsilon^{l\left(h_{k}\right)}\right)} & >\frac{1}{\prod_{i=0}^{\infty}\left(1+1 / q^{2 i+1}\right)} \\
& >\frac{2}{3} \cdot e^{-\sum_{i=1}^{\infty} 1 / q^{2 i+1}} \geqslant \frac{2 e^{-1 / 6}}{3} .
\end{aligned}
$$

Together with (i) and (5.2), the last two inequalities imply that

$$
\frac{\psi^{\lambda}(1) / q^{b(\lambda)}}{\psi^{\mu}(1) / q^{b(\mu)}} \geqslant \frac{15}{16} \cdot \frac{2 e^{-1 / 6}}{3}>\frac{1}{2},
$$

and so $\psi^{\lambda}(1) \geqslant q^{b(\lambda)-b(\mu)} / 2=q^{k(n-k)} / 2$. The same estimate holds if $n-1 \leqslant$ $k \leqslant n$.

Proposition 5.4. Let $\epsilon= \pm, G=\operatorname{GL}_{n}^{\epsilon}(q)$, and let $\chi \in \operatorname{Irr}(G)$ have level $0 \leqslant j \leqslant n$. Then the following statements hold:

(i) $\chi(1) \leqslant q^{n j}$.

(ii) $\chi(1) \geqslant q^{j(n-j)}$ if $\epsilon=+$ and $\chi(1) \geqslant q^{j(n-j)} / 2$ if $\epsilon=-$.

Proof. Since $\tau_{n}^{j}$ and $\zeta_{n}^{j}$ have degree $q^{n j}$, (i) follows from the definition of $\mathfrak{l}(\chi)$. Note that (ii) is obvious if $j \in\{0, n\}$, so we will assume $1 \leqslant j \leqslant n-1$. We can now apply Theorem 3.9 and Corollary 2.6 and see that $\chi= \pm R_{L}^{G}(\alpha \otimes \beta)$, where $L=G_{a}^{\epsilon} \times G_{b}^{\epsilon}$, where $0 \leqslant a \leqslant a+b=n, \alpha \in \operatorname{Irr}\left(G_{a}^{\epsilon}\right)$, and, up to a linear character, $\beta=\psi^{\lambda}$ for some $\lambda \vdash b$ with the longest part $\lambda_{1}=k=n-j$. In particular, using [DM2, Proposition 12.17], we have

$$
\chi(1)=[G: L]_{p^{\prime}} \alpha(1) \beta(1) \geqslant[G: L]_{p^{\prime}} \psi^{\lambda}(1) .
$$

If $a=0$ then we are done by Lemma 5.3(ii), (iii) (applied to $\psi^{\lambda}$ ). We will now assume that $a \geqslant 1$. If $\epsilon=+$, then by Lemmas 5.1(vi) and 5.3(ii) we have

$$
\chi(1) \geqslant q^{a b+k(b-k)} \geqslant q^{a k+k(b-k)}=q^{k(n-k)}=q^{j(n-j)} .
$$


Suppose that $\epsilon=-$. Applying Lemmas 5.1(vi) and 5.3(iii), we obtain that

$$
\chi(1) \geqslant(1 / 4) q^{a b+k(b-k)}=(1 / 4) q^{k(n-k)+a(b-k)} \geqslant q^{j(n-j)} / 2
$$

if $k<b$. If $k=b$, then $\beta(1)=q^{k(b-k)}$, so by Lemma 5.1(vi), we have

$$
\chi(1) \geqslant(1 / 2) q^{a b}=q^{j(n-j)} / 2 .
$$

Next, we aim to bound $\chi(1)$ from below when $\mathfrak{l}(\chi) \geqslant n / 2$. First, we begin with unipotent characters.

LEMMA 5.5. Let $\lambda=\left(k=\lambda_{1} \geqslant \lambda_{2} \geqslant \cdots \geqslant \lambda_{r} \geqslant 0\right) \vdash n$ and let $\psi=\psi^{\lambda} \in$ $\operatorname{Irr}\left(\mathrm{GL}_{n}^{\epsilon}(q)\right)$ for $n \geqslant 2$ and $\epsilon= \pm$. If $k \leqslant n / 2$, then $\psi(1) \geqslant q^{n^{2} / 4}$.

Proof. Note that if $k=1$, then $\psi$ is just the Steinberg character, of degree $q^{n(n-1) / 2}$, and the statement holds in this case. So we will assume $2 \leqslant k \leqslant n / 2$. Then Lemma 5.3(i) implies that

$$
2 b(\lambda)=2 \operatorname{deg}_{q}\left[G_{n}^{+}: G_{\lambda}^{+}\right]_{p^{\prime}}=n^{2}-\sum_{i=1}^{r} \lambda_{i}^{2}=[\lambda] / 2+n^{2} / 2 .
$$

Hence, Lemmas 5.3(ii) and 5.2 immediately imply $\psi(1) \geqslant q^{n^{2} / 4}$ in the case $\epsilon=$ + . We may now assume $\epsilon=-$. Since $(q+1) / q \leqslant 3 / 2<q^{0.6}$, by Lemma 5.3(iii) and (5.3), we have

$$
\psi(1)>q^{b(\lambda)-0.6(n-1)} \geqslant q^{n^{2} / 4}
$$

if $[\lambda] \geqslant 2.4(n-1)$. In particular, we are done if $[\lambda] \geqslant 2.4 n$. In the cases of exceptions to the latter inequality, as listed in Lemma 5.2, one can check using explicit formulas for $\psi^{\lambda}(1)$ (see [C, Section 13.8]) and estimates in Lemma 5.1 that $\psi(1) \geqslant q^{n^{2} / 4}$ as well.

We will need an extension of Lemma 5.5 in the case of unitary groups.

LEMMA 5.6. Let $G=\mathrm{GU}_{n}(q)$ with $n \geqslant 2$, and let $\chi \in \operatorname{Irr}(G)$ belong to the rational series $\mathcal{E}(G,(s))$, where all eigenvalues of the semisimple element $s \in G$ belong to $\mu_{q-\epsilon 1}$. Suppose that $\mathfrak{l}(\chi) \geqslant n / 2$. Then either $\chi(1)>q^{n^{2} / 4}$ or $\chi(1) \geqslant$ $(q-1) q^{n^{2} / 4-1}$ and one of the following cases occurs:

(i) $n=2 k, \mathbf{C}_{G}(s)=\mathrm{GU}_{k}(q) \times \mathrm{GU}_{k}(q)$, and $\chi(1)=\left[G: \mathbf{C}_{G}(s)\right]_{p^{\prime}}$.

(ii) $2 \leqslant n \leqslant 4$. 
Proof. (i) By the assumptions, we can decompose the natural module $V=\mathbb{F}_{q^{2}}^{n}$ into an orthogonal sum $\bigoplus_{i=1}^{m} V_{i}$, where the $V_{i}$ are distinct eigenspaces for $s$, say with eigenvalue $\epsilon_{i} \in \mu_{q-\epsilon 1}$. Setting $a_{i}:=\operatorname{dim}_{\mathbb{F}_{q^{2}}} V_{i}$ and $L:=\mathbf{C}_{G}(s)=$ $\mathrm{GU}\left(V_{1}\right) \times \cdots \times \mathrm{GU}\left(V_{m}\right)$, by (3.9), we then have

$$
\chi= \pm R_{L}^{G}\left(\alpha_{1} \otimes \alpha_{2} \otimes \cdots \otimes \alpha_{m}\right),
$$

where $\alpha_{i}=v_{i} \psi^{\lambda_{i}}, \lambda_{i} \vdash a_{i}$, and $v_{i}$ is a linear character of $\mathrm{GU}\left(V_{i}\right)=\mathrm{GU}_{a_{i}}(q)$. Let $k$ denote the largest among all the parts of all $\lambda_{i}$. Then $\mathfrak{l}(\chi) \geqslant n / 2$ implies that $k \leqslant n / 2$. By Lemma 5.5, we may assume that $m \geqslant 2$. One can check by direct computation that $\chi(1) \geqslant(q-1) q^{n^{2} / 4-1}$ when $2 \leqslant n \leqslant 4$, so we will assume that $n \geqslant 5$.

(ii) Here we consider the case $k=1$, that is, $\lambda_{i}=$ (1) for all $i$ and $m=n$. As $n \geqslant 5$, by Lemma 5.1(iv), we have

$$
\chi(1)=\left|\mathrm{GU}_{n}(q)\right|_{p^{\prime}} /(q+1)^{n}>q^{n(n+1) / 2-1.6 n}>q^{n^{2} / 4}
$$

(where we also used the trivial estimate $q+1<q^{1.6}$ ).

(iii) From now on, we may assume that $m, k \geqslant 2$. According to Lemma 5.3(iii), $\alpha_{i}(1) \geqslant(2 / 3)^{a_{i}-1} q^{b\left(\lambda_{i}\right)}$. Furthermore, by Lemma 5.1(iii), (iv),

$$
\left|\mathrm{GU}_{a_{i}}(q)\right|_{p^{\prime}} \leqslant(q+1) \prod_{j=2}^{a_{i}} q^{j} \leqslant(3 / 2) q^{a_{i}\left(a_{i}+1\right) / 2}, \quad\left|\mathrm{GU}_{n}(q)\right|_{p^{\prime}}>q^{n(n+1) / 2} .
$$

It follows that

$$
[G: L]_{p^{\prime}}>(2 / 3)^{m} q^{n(n+1) / 2-\sum_{i=1}^{m} a_{i}\left(a_{i}+1\right) / 2}=(2 / 3)^{m} q^{\left(n^{2}-\sum_{i=1}^{m} a_{i}^{2}\right) / 2} .
$$

Putting all these estimates together, we obtain

$$
\begin{aligned}
\chi(1) & =[G: L]_{p^{\prime}} \prod_{i=1}^{m} \alpha_{i}(1)>(2 / 3)^{m+\sum_{i=1}^{m}\left(a_{i}-1\right)} q^{\left(n^{2}-\sum_{i=1}^{m} a_{i}^{2}\right) / 2+\sum_{i=1}^{m} b\left(\lambda_{i}\right)} \\
& =(2 / 3)^{n} q^{b(\boldsymbol{\mu})}
\end{aligned}
$$

where the parts of the partition $\mu$ consist of all parts of all $\lambda_{i}$, put together in decreasing order. Note that $b(\boldsymbol{\mu})=\left([\boldsymbol{\mu}]+n^{2}\right) / 4$, and $q^{0.6}>3 / 2$. It follows that $\chi(1)>q^{n^{2} / 4}$ if $[\mu] \geqslant 2.4 n$. Thus, it remains to consider the exceptions to the latter inequality, listed in Lemma 5.2.

(iv) Consider the case $\boldsymbol{\mu}=(k, k, 1)$. If $m=3$, then (5.4) implies that

$$
\begin{aligned}
\chi(1) & =\left[\mathrm{GU}_{2 k+1}(q):\left(\mathrm{GU}_{k}(q) \times \mathrm{GU}_{k}(q) \times \mathrm{GU}_{1}(q)\right)\right]_{p^{\prime}} \\
& >(2 / 3)^{3} q^{n(n+1) / 2-k(k+1)-1}>q^{k^{2}+2 k-2}>q^{n^{2} / 4}
\end{aligned}
$$


if $k \geqslant 3$. It is easy to check that $\chi(1)>q^{n^{2} / 4}$ also holds when $k=2$.

Suppose $m=2$. If $\left\{\lambda_{1}, \lambda_{2}\right\}=\{(k, k),(1)\}$, then by Lemma 5.1(vi), we have

$$
\begin{aligned}
\chi(1) & =\frac{q^{k} \prod_{i=k+2}^{2 k}\left(q^{i}-(-1)^{i}\right)}{\prod_{i=2}^{k}\left(q^{i}-(-1)^{i}\right)} \cdot \frac{q^{2 k+1}+1}{q+1} \\
& =\frac{q^{k} \prod_{i=k+2}^{2 k+1}\left(q^{i}-(-1)^{i}\right)}{\prod_{i=1}^{k}\left(q^{i}-(-1)^{i}\right)} \geqslant \frac{q^{k(k+1)+k}}{2}>q^{n^{2} / 4} .
\end{aligned}
$$

If $\left\{\lambda_{1}, \lambda_{2}\right\}=\{(k, 1),(k)\}$, then by Lemma 5.1(vi), we have

$$
\chi(1)=\frac{\prod_{i=k+2}^{2 k+1}\left(q^{i}-(-1)^{i}\right)}{\prod_{i=1}^{k}\left(q^{i}-(-1)^{i}\right)} \cdot \frac{q^{k+1}-(-1)^{k} q}{q+1} \geqslant \frac{q^{k(k+1)+k}}{4}>q^{n^{2} / 4}
$$

when $k \geqslant 3$. It is easy to check that $\chi(1)>q^{n^{2} / 4}$ also holds when $k=2$.

(v) Next suppose that $\boldsymbol{\mu}=(k, k)$. Since $m \geqslant 2$, we have $\lambda_{1}=\lambda_{2}=(k)$ and so

$$
\chi(1)=\left[\mathrm{GU}_{2 k}(q):\left(\mathrm{GU}_{k}(q) \times \mathrm{GU}_{k}(q)\right)\right]_{p^{\prime}}>(q-1) q^{n^{2} / 4-1}
$$

by Lemma 5.1(vi). In fact, if the unipotent character $\psi$ of $\mathbf{C}_{G}(s)$ corresponding to $\chi$ is not the principal character, then $q \mid \psi(1)$ and so $\chi(1)>q^{n^{2} / 4}$.

(vi) Here we consider the case $\boldsymbol{\mu}=(k, k-1,1)$. Direct computations show that $\chi(1) \geqslant(q-1) q^{n^{2} / 4-1}$ if $k=2$. So we will assume $k \geqslant 3$. If $m=3$, then (5.4) implies that

$$
\begin{aligned}
\chi(1) & =\left[\mathrm{GU}_{2 k}(q):\left(\mathrm{GU}_{k}(q) \times \mathrm{GU}_{k-1}(q) \times \mathrm{GU}_{1}(q)\right)\right]_{p^{\prime}} \\
& >(2 / 3)^{3} q^{k(2 k+1)-k^{2}-1}>q^{k^{2}+k-3} \geqslant q^{n^{2} / 4} .
\end{aligned}
$$

Suppose $m=2$. If $\left\{\lambda_{1}, \lambda_{2}\right\}=\{(k, k-1),(1)\}$, then by Lemma 5.1(vi), we have

$$
\begin{aligned}
\chi(1) & =\frac{q^{k-1}\left(q^{2}-1\right) \prod_{i=k+2}^{2 k-1}\left(q^{i}-(-1)^{i}\right)}{\prod_{i=1}^{k-1}\left(q^{i}-(-1)^{i}\right)} \cdot \frac{q^{2 k}-1}{q+1} \\
& =\frac{q^{k-1}(q-1) \prod_{i=k+2}^{2 k}\left(q^{i}-(-1)^{i}\right)}{\prod_{i=1}^{k-1}\left(q^{i}-(-1)^{i}\right)} \geqslant q^{k^{2}+k-3} \geqslant q^{n^{2} / 4} .
\end{aligned}
$$

If $\left\{\lambda_{1}, \lambda_{2}\right\}=\{(k, 1),(k-1)\}$, then by Lemma 5.1(vi), we have

$$
\chi(1)=\frac{\prod_{i=k+2}^{2 k}\left(q^{i}-(-1)^{i}\right)}{\prod_{i=1}^{k-1}\left(q^{i}-(-1)^{i}\right)} \cdot \frac{q^{k+1}-(-1)^{k} q}{q+1} \geqslant q^{k^{2}+k-3} \geqslant q^{n^{2} / 4} .
$$


If $\left\{\lambda_{1}, \lambda_{2}\right\}=\{(k-1,1),(k)\}$, then by Lemma 5.1(vi), we have

$$
\chi(1)=\frac{\prod_{i=k+1}^{2 k}\left(q^{i}-(-1)^{i}\right)}{\prod_{i=1}^{k}\left(q^{i}-(-1)^{i}\right)} \cdot \frac{q^{k}+(-1)^{k} q}{q+1} \geqslant q^{k^{2}+k-3} \geqslant q^{n^{2} / 4} .
$$

(vii) Finally, one can check by direct computations that $\chi(1)>q^{n^{2} / 4}$ in the remaining cases, where $\boldsymbol{\mu}=(4,2,2),(3,2,2),(3,1,1,1),(2,2,2)$, and $(2,1$, $1,1)$.

LeMmA 5.7. Let $n=a+b$ with $a \in \mathbb{Z}_{\geqslant 2}, b \in \mathbb{Z}_{\geqslant 1}, \epsilon= \pm$, and let

$$
\gamma= \pm R_{\mathrm{GL}_{a}^{\epsilon}(q) \times \mathrm{GL}_{b}^{\epsilon}(q)}^{\mathrm{GL}_{\epsilon}^{\epsilon}(q)}(\alpha \otimes \beta),
$$

where $\alpha$ is a character of $\mathrm{GL}_{a}^{\epsilon}(q), \beta$ is a character of $\mathrm{GL}_{b}^{\epsilon}(q)$, and the sign for $\gamma$ is chosen so that $\gamma(1)>0$. Then $\gamma(1)>q^{(a+b)^{2} / 4}$ if at least one of the following conditions holds:

(i) $\epsilon=+, \alpha(1) \geqslant(9 / 16)(q-1) q^{a^{2} / 4-1}$, and $\beta(1) \geqslant q^{b^{2} / 4-2}$. Moreover, $\alpha(1) \geqslant$ $q-1$ if $(a, b)=(2,1)$.

(ii) $\epsilon=+, \alpha(1) \geqslant q^{a^{2} / 4-2}, b \geqslant 2$, and $\beta(1) \geqslant q^{k(b-k)}$ for some $k \in \mathbb{Z}$ with $b / 2 \leqslant k \leqslant \min \{b,(a+b) / 2\}$.

(iii) $\epsilon=-, \alpha(1) \geqslant q^{a^{2} / 4}$, and $\beta(1) \geqslant q^{k(b-k)} / 2$ with $k \in \mathbb{Z}$ and $b / 2 \leqslant k \leqslant$ $\min \{b,(a+b) / 2\}$.

(iv) $\epsilon=-, \alpha(1) \geqslant q^{a^{2} / 4}$, and $\beta(1) \geqslant q^{b^{2} / 4-1}$.

Proof. (i) By Lemma 5.1(vi), $\left[\mathrm{GL}_{n}^{+}:\left(\mathrm{GL}_{a}^{+} \times \mathrm{GL}_{b}^{+}\right)\right]_{p^{\prime}}>q^{a b}$. Also, $\alpha(1)>$ $q^{a^{2} / 4-2}$. Hence, if $a b \geqslant 8$, we have

$$
\gamma(1)>q^{a^{2} / 4+b^{2} / 4+a b-4} \geqslant q^{(a+b)^{2} / 4} .
$$

Consider the case $a b<8$. If $a=2$ and $b \geqslant 3$, then since $\alpha(1) \geqslant 1=q^{a^{2} / 4-1}$, we have

$$
\gamma(1)>q^{a^{2} / 4+b^{2} / 4+a b-3} \geqslant q^{(a+b)^{2} / 4} .
$$

The same argument applies if $a \geqslant 3$ and $b \geqslant 2$. If $a=b=2$, then

$$
\gamma(1)>q^{a b}=q^{(a+b)^{2} / 4} .
$$

We may now assume that $b=1$. If $a \geqslant 3$, then

$$
\left[\mathrm{GL}_{n}^{+}:\left(\mathrm{GL}_{a}^{+} \times \mathrm{GL}_{b}^{+}\right)\right]_{p^{\prime}}=\left(q^{a+1}-1\right) /(q-1) \geqslant \frac{15}{16} q^{a+1} /(q-1),
$$


whence

$$
\gamma(1) \geqslant \frac{9(q-1)}{16} q^{a^{2} / 4-1} \cdot \frac{15}{16(q-1)} q^{a+1}>q^{a^{2} / 4+a-1}>q^{(a+1)^{2} / 4} .
$$

If $a=2$, then $\gamma(1) \geqslant q^{3}-1>q^{9 / 4}$.

(ii) As in (i), note that $\gamma(1) / q^{(a+b)^{2} / 4}>q^{A}$, where

$$
A:=\frac{a^{2}}{4}-2+k(b-k)+a b-\frac{(a+b)^{2}}{4} \geqslant \frac{1}{4}((2 k-b)(3 b-2 k)-8)
$$

as $a \geqslant 2 k-b$. In particular, $A>1$, and so we so are done, if $k=b \geqslant 3$. If $k=b=2$ but $a \geqslant 3$, then $A=a-3 \geqslant 0$. If $k=b=a=2$, then we have $\gamma(1) \geqslant q^{a b}=q^{(a+b)^{2} / 4}$.

So we may assume $k \leqslant b-1$, and so $3 b-2 k \geqslant b+2 \geqslant 4$. If $2 k-b \geqslant 2$, then $A \geqslant 0$. If $2 k-b=0$, then $A=(a b-4) / 2 \geqslant 0$. If $2 k-b=1$, then $b \geqslant 3$ and $A=a b-9 / 2 \geqslant 3 / 2$.

(iii) First we consider the case $b=1$ and use the trivial bound $\beta(1) \geqslant 1$. If $a=2$, then $\gamma(1) \geqslant q\left(q^{2}-q+1\right)>q^{9 / 4}$. If $a \geqslant 3$, then

$$
\gamma(1) \geqslant \frac{q^{a+1}-(-1)^{a+1}}{q+1} q^{a^{2} / 4}>q^{a^{2} / 4+a-1}>q^{(a+1)^{2} / 4} .
$$

If $k=a=b=2$, then $\gamma(1) \geqslant q\left(q^{2}+1\right)\left(q^{2}-q+1\right)>q^{4}$. Now we may assume that $b \geqslant 2$ and $(k, a, b) \neq(2,2,2)$. By Lemma 5.1(vi),

$$
\left[\mathrm{GL}_{n}^{-}:\left(\mathrm{GL}_{a}^{-} \times \mathrm{GL}_{b}^{-}\right)\right]_{p^{\prime}} \geqslant(5 / 8) q^{a b}>q^{a b-1}
$$

and $\beta(1) \geqslant q^{k(b-k)-1}$. It follows that $\gamma(1) / q^{(a+b)^{2} / 4}>q^{A}$, where $A$ is defined in (5.5). As shown in (ii), $A \geqslant 0$, and so we are done.

(iv) The case $b=1$ follows from the same arguments as in (iii). Suppose $b \geqslant 2$. As in (iii), we now have $\gamma(1) \geqslant q^{a^{2} / 4+b^{2} / 4+a b-2} \geqslant q^{(a+b)^{2} / 4}$.

LEMMA 5.8. Let $n=m d$ with $m \in \mathbb{Z}_{\geqslant 1}$ and $d \in \mathbb{Z}_{\geqslant 2}$.

(i) If $d=2$, then $\left[\mathrm{GL}_{n}(q): \mathrm{GL}_{m}\left(q^{d}\right)\right]_{p^{\prime}}>(9 / 16)(q-1) q^{n^{2} / 4-1}$.

(ii) If $d \geqslant 3$, then $\left[\mathrm{GL}_{n}(q): \mathrm{GL}_{m}\left(q^{d}\right)\right]_{p^{\prime}}>q^{n^{2} / 4}$ unless $(n, d, q)=(3,3,2)$ in which case $\left[\mathrm{GL}_{n}(q): \mathrm{GL}_{m}\left(q^{d}\right)\right]_{p^{\prime}}>(q-1) q^{n^{2} / 4-1}$.

(iii) $\left[\mathrm{GU}_{n}(q): \mathrm{GL}_{m}\left(q^{d}\right)\right]_{p^{\prime}}>q^{n^{2} / 4}$ if $2 \mid d$.

(iv) $\left[\mathrm{GU}_{n}(q): \mathrm{GU}_{m}\left(q^{d}\right)\right]_{p^{\prime}}>(1.49) q^{n^{2} / 4}$ if $2 \nmid d$. 
Proof. (i) By Lemma 5.1(i), we have

$$
\begin{aligned}
{\left[\mathrm{GL}_{n}(q): \mathrm{GL}_{m}\left(q^{d}\right)\right]_{p^{\prime}} } & =\prod_{i=1}^{m}\left(q^{2 i-1}-1\right)>\frac{9}{16} \cdot \frac{q-1}{q} q^{\sum_{i=1}^{m}(2 i-1)} \\
& =\frac{9}{16}(q-1) q^{n^{2} / 4-1}
\end{aligned}
$$

(ii) The statement can be checked directly if $n \leqslant 4$, so we will assume that $n \geqslant 5$. Now by Lemma 5.1(ii), we have

$$
\begin{aligned}
& \left.\operatorname{LGL}_{n}(q): \operatorname{GL}_{m}\left(q^{d}\right)\right]_{p^{\prime}} \\
& \quad=\prod_{i=1, d \nmid i}^{n}\left(q^{i}-1\right)>\frac{9}{16} \cdot \frac{q-1}{q} q^{\sum_{i=1, d \nmid i}^{n} i}>q^{n^{2} / 2-n^{2} / 2 d-2}>q^{n^{2} / 4} .
\end{aligned}
$$

(iii) Here we have

$$
\left[\mathrm{GU}_{n}(q): \mathrm{GL}_{m}\left(q^{d}\right)\right]_{p^{\prime}} \geqslant\left[\mathrm{GU}_{n}(q): \mathrm{GL}_{n / 2}\left(q^{2}\right)\right]_{p^{\prime}}=\prod_{i=1}^{n / 2}\left(q^{2 i-1}+1\right)>q^{n^{2} / 4} \text {. }
$$

(iv) By Lemma 5.1(iii), we have

$$
\begin{aligned}
\left|\mathrm{GU}_{m}\left(q^{d}\right)\right|_{p^{\prime}} & =\left(q^{d}+1\right) \prod_{i=2}^{m}\left(q^{d i}-(-1)^{i}\right) \leqslant\left(q^{d}+1\right) \prod_{i=2}^{m} q^{d i} \\
& \leqslant \frac{9}{8} \prod_{i=1}^{m} q^{d i}=\frac{9}{8} q^{m d(m+1) / 2} .
\end{aligned}
$$

It then follows from Lemma 5.1(iv) that

$$
\begin{aligned}
{\left[\mathrm{GU}_{n}(q): \mathrm{GU}_{m}\left(q^{d}\right)\right]_{p^{\prime}}>\frac{q^{n(n+1) / 2}}{(9 / 8) q^{m d(d+1) / 2}} } & =\frac{8}{9} q^{n^{2} / 2-n^{2} / 2 d} \\
& \geqslant \frac{8}{9} q^{n^{2} / 3}>(1.49) q^{n^{2} / 4} .
\end{aligned}
$$

Recall the notation (3.12) for irreducible characters of $\mathrm{GL}_{n}(q)$.

Proposition 5.9. Let $n \geqslant 2$ and let $\chi \in \operatorname{Irr}\left(\mathrm{GL}_{n}(q)\right)$ be of level $\mathfrak{l}(\chi) \geqslant n / 2$. Then either $\chi(1) \geqslant q^{n^{2} / 4}$ or one of the following statements holds:

(i) $\chi=S(s,(n / 2))$ with $\operatorname{deg}(s)=2$, and

$$
\chi(1)=\prod_{i=1}^{n / 2}\left(q^{2 i-1}-1\right)>(9 / 16)(q-1) q^{n^{2} / 4-1} .
$$


(ii) $(n, q)=(3,2), \chi=S(s,(1))$ with $\operatorname{deg}(s)=3$, and $\chi(1)=3>(q-$ 1) $q^{n^{2} / 4-1}$.

Proof. (a) We represent $\chi$ in the form (3.12), where $d_{1} \geqslant d_{2} \geqslant \cdots \geqslant d_{m} \geqslant 1$. First, we consider the case $m=1$ and $d=d_{1} \geqslant 2$. If $d \geqslant 3$, or $d=2$ and $\lambda_{1}=$ $(n / 2)$, then the statement follows from Lemma 5.8(i), (ii). Suppose $d=2$, but $\lambda_{1} \neq(n / 2)$. Then the unipotent character $\psi$ of $\mathrm{GL}_{n / 2}\left(q^{2}\right)$ in (3.8) corresponding to $\chi$ has degree divisible by $q^{2}$, whence using Lemma 5.8(i), we have

$$
\chi(1)>\frac{9}{16}(q-1) q^{n^{2} / 4-1} \cdot q^{2}>q^{n^{2} / 4} .
$$

We have also shown that

$$
\operatorname{deg} S\left(s_{i}, \lambda_{i}\right) \geqslant \frac{9}{16}(q-1) q^{k_{i}^{2} d_{i}^{2} / 4-1}
$$

if $d_{i}>1$.

(b) Here we consider the case $d_{1}=1$, and let $k$ denote the largest among all the parts of all $\lambda_{i}, 1 \leqslant i \leqslant m$. Since $\mathfrak{l}(\chi) \geqslant n / 2$, we must have by Theorem 3.9 that $k \leqslant n / 2$. By Lemma 5.3(ii), (iv), we have $\chi(1) \geqslant q^{N}$, where

$$
N:=\operatorname{deg}_{q}\left[G_{n}^{+}:\left(G_{k_{1}}^{+} \times \cdots \times G_{k_{m}}^{+}\right)\right]_{p^{\prime}}+\sum_{i=1}^{m} b\left(\lambda_{i}\right)=b(\boldsymbol{\mu}),
$$

and the partitions of $\boldsymbol{\mu} \vdash n$ consist of all parts of all $\lambda_{i}$, put in decreasing order; in particular, the longest part $\mu_{1}$ of $\boldsymbol{\mu}$ is $k$. Hence, $b(\boldsymbol{\mu}) \geqslant n^{2} / 4$, as shown in the proof of Lemma 5.5.

(c) We may now assume that $m \geqslant 2, d_{1} \geqslant \cdots \geqslant d_{t} \geqslant 2$ for some $1 \leqslant t \leqslant m$; and, furthermore, $d_{t+1}=\cdots=d_{m}=1$ if $t<m$, in which case we let $k$ denote the largest among all the parts of all $\lambda_{i}, t+1 \leqslant i \leqslant m$. Also, set $a:=\sum_{i=1}^{t} k_{i} d_{i}$ and $b:=\sum_{i=t+1}^{m} k_{i}$. Then $\chi=R_{\mathrm{GL}_{a} \times \mathrm{GL}_{b}}^{\mathrm{GL}_{n}}(\alpha \otimes \beta)$, where

$$
\alpha:=S\left(s_{1}, \lambda_{1}\right) \circ \cdots \circ S\left(s_{t}, \lambda_{t}\right), \quad \beta:=S\left(s_{t+1}, \lambda_{t+1}\right) \circ \cdots \circ S\left(s_{m}, \lambda_{m}\right) .
$$

Note that $\operatorname{deg} S\left(s_{i}, \lambda_{i}\right)=q-1$ if $\left(k_{i}, d_{i}\right)=(1,2)$; in particular, $\alpha(1) \geqslant q-1$. Applying (5.6) and Lemma 5.7(i), we get $\alpha(1) \geqslant(9 / 16)(q-1) q^{a^{2} / 4-1}$ if $t=1$ and $\alpha(1)>q^{a^{2} / 4}$ if $t \geqslant 2$. In particular, we are done if $t=m$.

We may now assume that $t<m$ and $\alpha(1) \geqslant \max \left\{(9 / 16)(q-1) q^{a^{2} / 4-1}, q-1\right\}$. If $k \leqslant b / 2$ (in particular, $b \geqslant 2$ ), then $\beta(1) \geqslant q^{b^{2} / 4}$ as shown in (b), whence $\chi(1)>q^{n^{2} / 4}$ by Lemma 5.7(i). Finally, suppose that $b \geqslant k>b / 2$. Since ll $(\chi) \geqslant$ $n / 2$, we again have that $k \leqslant n / 2$. Also, $\beta(1) \geqslant q^{k(b-k)}$ by Proposition 5.4(ii). It follows, by Lemma 5.7(i) for $b=1$ and by Lemma 5.7(ii) for $b \geqslant 2$, that $\chi(1)>q^{n^{2} / 4}$. 
Proposition 5.10. Let $n \geqslant 2$ and let $\chi \in \operatorname{Irr}\left(\mathrm{GU}_{n}(q)\right)$ be of level $\mathfrak{l}(\chi) \geqslant n / 2$. Then either $\chi(1) \geqslant q^{n^{2} / 4}$ or $\chi(1) \geqslant(q-1) q^{n^{2} / 4-1}$ and one of the following cases occurs:

(i) $n=2 k, \mathbf{C}_{G}(s)=\mathrm{GU}_{k}(q) \times \mathrm{GU}_{k}(q)$, and $\chi(1)=\left[G: \mathbf{C}_{G}(s)\right]_{p^{\prime}}$.

(ii) $2 \leqslant n \leqslant 4$.

Proof. Let $\chi \in \mathcal{E}(G,(s))$ where $s \in G$ is semisimple. We will represent $\chi$ in the form of (3.9) and (3.8). Let $a:=\operatorname{dim}_{\mathbb{F}_{q^{2}}} V^{0}$ and $b:=\operatorname{dim}_{\mathbb{F}_{q^{2}}} V^{1}$.

(a) Suppose that $b>0$. Then we can decompose $V^{1}$ into an orthogonal sum of $s$-invariant nondegenerate subspaces, say $V_{j}$ of dimension $b_{j}$ over $\mathbb{F}_{q^{2}}, 1 \leqslant j \leqslant t$, and $b=\sum_{j=1}^{t} b_{j}$, in such a way that $\mathbf{C}_{\mathrm{GU}\left(V_{j}\right)}(s)$ is either $\mathrm{GL}_{b_{j} / d_{j}}\left(q^{d_{j}}\right)$ with $2 \mid d_{j}$ or $\mathrm{GU}_{b_{j} / d_{j}}\left(q^{d_{j}}\right)$ with $2 \nmid d_{j}>1$. By Lemma 5.8(iii), (iv),

$$
\left[\mathrm{GU}\left(V_{j}\right): \mathbf{C}_{\mathrm{GU}\left(V_{j}\right)}(s)\right]_{p^{\prime}}>q^{b_{j}^{2} / 4},
$$

and, furthermore, $b_{j} \geqslant 2$. Now, a repeated application of Lemma 5.7(iv) using (5.7) shows that

$$
\left[\mathrm{GU}\left(V^{1}\right): \mathbf{C}_{\mathrm{GU}\left(V^{1}\right)}(s)\right]_{p^{\prime}}>q^{b^{2} / 4} .
$$

In particular, $\chi(1)>q^{n^{2} / 4}$ if $a=0$.

(b) If $b=0$, then we are done by Lemma 5.6. So we will assume that $a, b>0$. Let $k$ denote the largest among all the parts of the partitions $\boldsymbol{\gamma}_{\delta}, \delta \in \mu_{q+1}$. Since $\mathfrak{l}(\chi) \geqslant n / 2$, we have $k \leqslant n / 2$ by Theorem 3.9. By Lemma 2.5(ii), we can also write

$$
\chi= \pm R_{\mathrm{GU}_{a}(q) \times \mathrm{GU}_{b}(q)}^{\mathrm{GU}_{n}(\alpha)}(\alpha \otimes \beta),
$$

where

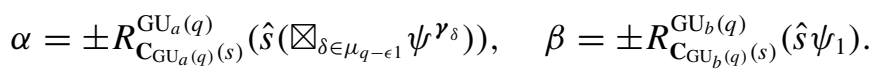

Now $\alpha(1) \geqslant q^{k(a-k)} / 2$ by Proposition 5.4 , and $\beta(1)>q^{b^{2} / 4}$ by (5.8); also, $b \geqslant 2$. Applying Lemma 5.7(iii), we obtain that $\chi(1)>q^{n^{2} / 4}$ if $k \geqslant a / 2$. If $k<a / 2$, then $\alpha(1) \geqslant q^{a^{2} / 4-1}$ by Lemma 5.6, whence $\chi(1)>q^{n^{2} / 4}$ by Lemma 5.7(iv).

Now we can prove the main result of this section, Theorem 1.2, which we restate below.

THEOREM 5.11. Let $n \geqslant 2, \epsilon= \pm$, and $G=\operatorname{GL}_{n}^{\epsilon}(q)$. Set $\kappa_{+}=1$ and $\kappa_{-}=1 / 2$. Let $\chi \in \operatorname{Irr}(G)$ have level $j=\mathfrak{l}(\chi)$. Then the following statements hold:

(i) $\kappa_{\epsilon} q^{j(n-j)} \leqslant \chi(1) \leqslant q^{n j}$. 
(ii) If $j \geqslant n / 2$, then

$$
\chi(1)>(9 / 16)(q-1) q^{n^{2} / 4-1}
$$

if $\epsilon=+$, and

$$
\chi(1) \geqslant(q-1) q^{n^{2} / 4-1}
$$

if $\epsilon=-$. In particular, $\chi(1)>q^{n^{2} / 4-2}$ if $\mathfrak{l}(\chi) \geqslant n / 2$.

(iii) If $n \geqslant 7$ and $\left\lceil(1 / n) \log _{q} \chi(1)\right\rceil<\sqrt{n-1}-1$, then

$$
\mathfrak{l}(\chi)=\left\lceil\frac{\log _{q} \chi(1)}{n}\right\rceil .
$$

Proof. (i) is Proposition 5.4, and (ii) follows from Propositions 5.9 and 5.10. For (iii), we have that $j_{0}:=\left\lceil(1 / n) \log _{q} \chi(1)\right\rceil<\sqrt{n-1}-1$ by hypothesis. In particular,

$$
\chi(1) \leqslant q^{n j_{0}}<q^{n(\sqrt{n-1}-1)}<q^{n^{2} / 4-2}
$$

as $n \geqslant 7$. It then follows from (ii) that $j<n / 2$. Also, $\chi(1) \leqslant q^{n j}$ by (i), whence $(1 / n) \log _{q} \chi(1) \leqslant j$ and so $j \geqslant j_{0}$. Suppose that $j>j_{0}$. Then $j_{0}+1 \leqslant j<n / 2$ and $j_{0}<\sqrt{n-1}-1$, and so

$$
j(n-j) \geqslant\left(j_{0}+1\right)\left(n-j_{0}-1\right)=n j_{0}+n-\left(j_{0}+1\right)^{2}>n j_{0}+1 .
$$

Combined with (i), this implies that $\chi(1) \geqslant q^{j(n-j)-1}>q^{n j_{0}}$, a contradiction.

COROLlary 5.12. Let $G=\mathrm{GL}_{n}^{\epsilon}(q)$ with $n \geqslant 2$ and $\epsilon= \pm$. For $\chi \in \operatorname{Irr}(G)$,

$$
\chi(1) \cdot\left|D_{\mathcal{G}}(\chi)(1)\right|>q^{n^{2} / 4-2} .
$$

Proof. The statement follows from Theorem 5.11(ii) if $\mathfrak{l}(\chi) \geqslant n / 2$ or $\mathfrak{l}\left( \pm D_{\mathcal{G}}(\chi)\right) \geqslant n / 2$. If $\mathfrak{l}(\chi), \mathfrak{l}\left( \pm D_{\mathcal{G}}(\chi)\right)<n / 2$, then by Proposition 4.4 , we have $\mathfrak{l}(\chi)=\mathfrak{l}\left( \pm D_{\mathcal{G}}(\chi)\right)=(n-1) / 2$, in which case by Theorem 5.11(i), we have

$$
\chi(1) \cdot\left|D_{\mathcal{G}}(\chi)(1)\right| \geqslant q^{\left(n^{2}-1\right) / 2-2}>q^{n^{2} / 4-2} .
$$

\section{Bounds on character values}

PROPOSITION 6.1. There is an explicit function

$$
f=f(C, m, k): \mathbb{R}_{\geqslant 1} \times \mathbb{Z}_{\geqslant-1} \times \mathbb{Z}_{\geqslant 0} \rightarrow \mathbb{R}_{\geqslant 1}
$$


such that, for any $C \in \mathbb{R}_{\geqslant 1}, m \in \mathbb{Z}_{\geqslant-1}, k \in \mathbb{Z}_{>0}$, the following property $\mathcal{A}(C$, $m, k)$ holds:

There exists some $\delta=\delta(C, m, k) \in\left[1 / 2^{m+1}, 1 / 2^{m}\right)$

such that, for any prime power $q$, any $\epsilon= \pm$,

$\mathcal{A}(C, m, k): \quad$ any $G:=\mathrm{GL}_{n}^{\epsilon}(q)$ with $n \geqslant f(C, m, k)$,

any $g \in G$ with $\left|\mathbf{C}_{G}(g)\right| \leqslant q^{C n}$, and any $\chi \in \operatorname{Irr}(G)$ of level $k$, $|\chi(g)| \leqslant \chi(1)^{\delta}$.

Proof. (i) We will prove $\mathcal{A}(C, m, k)$ by induction on $m \geqslant-1$. Certainly, we can take $f(C,-1, k)=1$ and $\delta(C,-1, k)=1$. For the induction step, assume $m \geqslant 0$ and consider any $g \in G$ with $\left|\mathbf{C}_{G}(g)\right| \leqslant q^{C n}$; in particular,

$$
|\chi(g)| \leqslant q^{C n / 2}, \forall \chi \in \operatorname{Irr}(G) .
$$

(ii) Now we consider any $n \geqslant N_{1}:=2^{m+2} C+8$. This implies that $n^{2} / 4-$ $2 \geqslant 2^{m} \mathrm{Cn}$. Hence, if $\mathfrak{l}(\chi)=j \geqslant n / 2$, then by Theorem 1.2(ii), we have that $\chi(1) \geqslant q^{2^{m} C n}$, and so

$$
|\chi(g)| \leqslant q^{C n / 2} \leqslant \chi(1)^{1 / 2^{m+1}} .
$$

Next suppose that $\mathfrak{l}(\chi)=j \geqslant 2^{m+1} C$ but $j<n / 2$. Then $2 \leqslant j \leqslant(n-1) / 2$, whence $j n / 2-j^{2}-1 \geqslant j / 2-1 \geqslant 0$. It follows by Theorem 1.2(i) that $\chi(1) \geqslant$ $q^{j(n-j)-1} \geqslant q^{j n / 2}$ and so we again have

$$
|\chi(g)| \leqslant q^{C n / 2} \leqslant \chi(1)^{1 / 2^{m+1}} .
$$

Thus, $\mathcal{A}(C, m, j)$ holds for all $n \geqslant N_{1}$ and all $j \geqslant 2^{m+1} C$, by taking $\delta(C, m$, $j)=1 / 2^{m+1}$.

(iii) We will now prove $\mathcal{A}(C, m, k)$ by backward induction on $k \geqslant 0$ assuming $n \geqslant 2 N_{1}, k<2^{m+1} C$, and may therefore assume that $\mathcal{A}(C, m, j)$ holds for any $j$ with $k+1 \leqslant j \leqslant 2 k$ as well as that $\mathcal{A}(C, m-1, j)$ holds for all $j \geqslant 0$.

Consider any $\chi \in \operatorname{Irr}(G)$ with $\mathfrak{l}(\chi)=k$. If $k=0$, then $\chi(1)=1$ by Theorem 1.2(i) and so we can take any $\delta \geqslant 1 / 2^{m+1}$. So we will assume $k \geqslant 1$. Let $V=\mathbb{F}_{q}^{n}$, respectively $V=\mathbb{F}_{q^{2}}^{n}$, denote the natural module for $G$. By the definition of the level, $\lambda \chi$ is an irreducible constituent of $\sigma^{k}$ for some linear character $\lambda$ of $G$, where $\sigma:=\tau_{n}$, respectively $\zeta_{n}$. As $\sigma=\bar{\sigma}$, we see that $\sigma^{2 k}=\chi \bar{\chi}+\rho$, where either $\rho=0$ or $\rho$ is a $G$-character. Writing

$$
\chi \bar{\chi}=\sum_{i=1}^{t} a_{i} \chi_{i},
$$


where $\chi_{i} \in \operatorname{Irr}(G)$ and $a_{i} \in \mathbb{Z}_{>0}$, we have

$$
\sum_{i=1}^{t} a_{i} \leqslant \sum_{i=1}^{t} a_{i}^{2}=[\chi \bar{\chi}, \chi \bar{\chi}]_{G} \leqslant\left[\sigma^{2 k}, \sigma^{2 k}\right]_{G}=\left[\sigma^{4 k}, 1_{G}\right]_{G} .
$$

If $\epsilon=+$, then $\left[\sigma^{4 k}, 1_{G}\right]_{G}$ is just the number of $G$-orbits on $\underbrace{V \times V \times \cdots \times V}_{4 k}$, and $k \leqslant n / 4$ by our assumptions on $n, k$; hence, it is at most $8 q^{4 k^{2}}$ by Lemma 2.4. If $\epsilon=-$, then $\left[\sigma^{4 k}, 1_{G}\right]_{G}$ is the number of $G$-orbits on $\underbrace{V \times V \times \cdots \times V}_{2 k}$, whence it is at most $2 q^{4 k^{2}}$ by Lemma 2.4. We have, therefore, shown that

$$
|\chi(g)|^{2} \leqslant q^{4 k^{2}+3} \max _{1 \leqslant i \leqslant t}\left|\chi_{i}(g)\right| .
$$

Since $\chi_{i}$ is an irreducible constituent of $\sigma^{2 k}, \mathfrak{l}\left(\chi_{i}\right) \leqslant 2 k$. If $0 \leqslant \mathfrak{l}\left(\chi_{i}\right) \leqslant k$, then by taking

$n \geqslant N_{2}:=\max _{0 \leqslant j \leqslant k} f(C, m-1, j), \quad \alpha:=\max _{0 \leqslant j \leqslant k} \delta(C, m-1, j) \in\left[1 / 2^{m}, 1 / 2^{m-1}\right)$, we have by $\mathcal{A}(C, m-1, j), 0 \leqslant j \leqslant k$, and Theorem 1.2(i) that

$$
\left|\chi_{i}(g)\right| \leqslant\left|\chi_{i}(1)\right|^{\alpha} \leqslant q^{k n \alpha} .
$$

On the other hand, if $k<\mathfrak{l}\left(\chi_{i}\right) \leqslant 2 k$, then by taking

$$
n \geqslant N_{3}:=\max _{k<j \leqslant 2 k} f(C, m, j), \quad \beta:=\max _{k<j \leqslant 2 k} \delta(C, m, j) \in\left[1 / 2^{m+1}, 1 / 2^{m}\right),
$$

we have by $\mathcal{A}(C, m, j), k<j \leqslant 2 k$, and Theorem 1.2(i) that

$$
\left|\chi_{i}(g)\right| \leqslant\left|\chi_{i}(1)\right|^{\beta} \leqslant q^{2 k n \beta} .
$$

It follows from (6.1)-(6.3) that

$$
|\chi(g)| \leqslant q^{2 k^{2}+3 / 2} q^{k n \gamma},
$$

where $\gamma:=\max (\alpha / 2, \beta) \in\left[1 / 2^{m+1}, 1 / 2^{m}\right)$, if $n \geqslant \max \left(2 N_{1}, N_{2}, N_{3}\right)$. Now we choose $\delta=\delta(C, m, k)$ such that $\gamma<\delta<1 / 2^{m}$ and

$$
\begin{aligned}
n \geqslant f(C, m, k) & :=\max \left(2 N_{1}, N_{2}, N_{3}, \frac{3 k+3}{\delta-\gamma}\right) \\
& =\max \left(2^{m+3} C+16, \max _{0 \leqslant j \leqslant k} f(C, m-1, j),\right.
\end{aligned}
$$




$$
\left.\max _{k<j \leqslant 2 k} f(C, m, j), \frac{3 k+3}{\delta-\gamma}\right) .
$$

Then $\left(2 k^{2}+3 / 2+k n \gamma\right) \leqslant\left(k n-k^{2}-1\right) \delta$. Since $\chi(1) \geqslant q^{k n-k^{2}-1}$ by Theorem 1.2(i), (6.4) now implies that $|\chi(g)| \leqslant \chi(1)^{\delta}$, completing the induction step of the proof.

Now we can prove Theorem 1.4 for $\mathrm{GL}_{n}^{\epsilon}(q)$, which we restate below.

THEOREM 6.2. There is an explicit function $h=h(C, m): \mathbb{R}_{\geqslant 1} \times \mathbb{Z}_{\geqslant 0} \rightarrow \mathbb{R}_{\geqslant 1}$ such that, for any $C \in \mathbb{R}_{\geqslant 1}, m \in \mathbb{Z}_{\geqslant 0}$, the following statement holds. For any prime power $q$, any $\epsilon= \pm$, any $G:=\mathrm{GL}_{n}^{\epsilon}(q)$ with $n \geqslant h(C, m)$, any $g \in G$ with $\left|\mathbf{C}_{G}(g)\right| \leqslant q^{C n}$, and any $\chi \in \operatorname{Irr}(G)$,

$$
|\chi(g)| \leqslant \chi(1)^{1 / 2^{m}}
$$

Proof. Consider any $g \in G$ with $\left|\mathbf{C}_{G}(g)\right| \leqslant q^{C n}$. As shown in page (ii) of the proof of Proposition 6.1, $|\chi(g)| \leqslant \chi(1)^{1 / 2^{m}}$ if $n \geqslant 2^{m+1} C+8$ and $\mathfrak{l}(\chi) \geqslant 2^{m} C$. On the other hand, by Proposition 6.1, we have

$$
|\chi(g)|<\chi(1)^{1 / 2^{m}}
$$

if $\mathfrak{l}(\chi)=k<2^{m} C$ and $n \geqslant f(C, m, k)$. Thus, $|\chi(g)| \leqslant \chi(1)^{1 / 2^{m}}$ for all

$$
n \geqslant h(C, m):=\max \left(2^{m+1} C+8, \max _{1 \leqslant k<2^{m} C} f(C, m, k)\right) .
$$

Next we prove Theorem 1.5 for $\mathrm{GL}_{n}^{\epsilon}(q)$, which we restate below.

THEOREM 6.3. Let $q$ be any prime power and let $G=\mathrm{GL}_{n}^{\epsilon}(q)$ with $\epsilon= \pm$. Suppose that $g \in G$ satisfies $\left|\mathbf{C}_{G}(g)\right| \leqslant q^{n^{2} / 12}$. Then

$$
|\chi(g)| \leqslant \chi(1)^{8 / 9}
$$

for all $\chi \in \operatorname{Irr}(G)$.

Proof. (i) We will work with the assumption that $\left|\mathbf{C}_{G}(g)\right| \leqslant q^{n^{2} \delta}$ with $\delta=1 / 12$. (In fact, $\delta$ can also be chosen to be $35 / 418$.) Let $\mathfrak{l}(\chi)=k$, and we aim to show that

$$
|\chi(g)| \leqslant \chi(1)^{8 / 9} .
$$

(ii) Note that when $1 \leqslant n \leqslant 4$, there is no element $g \in G$ such that $\left|\mathbf{C}_{G}(g)\right| \leqslant q^{n^{2} / 12}$ and that the statement is trivial if $\chi(1)=1$. So we will assume 
that $n \geqslant 5$ and $\chi(1)>1$. Furthermore, if $k=1$, then $\chi$ is a Weil character; see Examples 3.11 and 3.12. In this case, it is not difficult to use character formulas for Weil characters, see, for example, [TZ, Lemma 4.1], to verify that the statement holds for $g$. So we will assume $k \geqslant 2$. It then follows by Theorem 1.2(i) that $\chi(1) \geqslant q^{2 n-5}$. In particular, if $3 \leqslant n \leqslant 41$, then $n^{2} / 24<(8 / 9)(2 n-5)$, and so

$$
|\chi(g)| \leqslant\left|\mathbf{C}_{G}(g)\right|^{1 / 2} \leqslant q^{n^{2} \delta / 2}=q^{n^{2} / 24}<\chi(1)^{8 / 9} .
$$

So we may assume

$$
n \geqslant 42, \quad k \geqslant 2 \text {. }
$$

Suppose that $k \geqslant n / 15$. Then Theorem 1.2 implies that $\chi(1) \geqslant q^{14 n^{2} / 225-1}$, whereas $|\chi(g)| \leqslant q^{n^{2} / 24}$ and so $|\chi(g)|<\chi(1)^{8 / 9}$. So we may assume $k<n / 15$, whence $k<n / 11-1$ because of (6.5). In this case, $k(n-k)-1>10 k n / 11$, and so Theorem 1.2(i) yields

$$
\chi(1) \geqslant q^{10 k n / 11} .
$$

Now, if $k \geqslant 5 n \delta / 8$, then $(8 / 9)(10 k n / 11)>n^{2} \delta / 2$ and so (6.6) implies that

$$
|\chi(g)| \leqslant\left|\mathbf{C}_{G}(g)\right|^{1 / 2} \leqslant q^{n^{2} \delta / 2}<\chi(1)^{8 / 9} .
$$

Thus, we may assume that

$$
k<5 n \delta / 8 \text {. }
$$

(iii) For some integer $1 \leqslant m \leqslant n / 4 k$, to be chosen later, we decompose

$$
(\chi \bar{\chi})^{m}=\sum_{i=1}^{s} a_{i} \alpha_{i}+\sum_{j=1}^{t} b_{j} \beta_{j},
$$

where $a_{i}, b_{j} \in \mathbb{Z}_{>0}, \alpha_{i} \in \operatorname{Irr}(G), \beta_{j} \in \operatorname{Irr}(G)$, and

$$
\alpha_{i}(1)<q^{n^{2} \delta}, \quad \beta_{j}(1) \geqslant q^{n^{2} \delta} .
$$

Arguing as in page (iii) of the proof of Proposition 6.1 and using the condition $4 \mathrm{~km} \leqslant n$, we obtain

$$
\sum_{i=1}^{s} a_{i}+\sum_{j=1}^{t} b_{j} \leqslant q^{4 m^{2} k^{2}+3} \leqslant q^{19 m^{2} k^{2} / 4}
$$

as $k \geqslant 2$. Using the bounds $\left|\alpha_{i}(g)\right| \leqslant \alpha_{i}(1)$ and

$$
\left|\beta_{j}(g)\right| \leqslant\left|\mathbf{C}_{G}(g)\right|^{1 / 2} \leqslant q^{n^{2} \delta / 2} \leqslant \beta_{j}(1) / q^{n^{2} \delta / 2},
$$


we have

$$
\begin{gathered}
\left|\sum_{i=1}^{s} a_{i} \alpha_{i}(g)\right| \leqslant \sum_{i=1}^{s} a_{i} \alpha_{i}(1) \leqslant q^{19 m^{2} k^{2} / 4+n^{2} \delta}, \\
\left|\sum_{j=1}^{t} b_{j} \beta_{j}(g)\right| \leqslant \frac{\sum_{j=1}^{t} b_{j} \beta_{j}(1)}{q^{n^{2} \delta / 2}} \leqslant \frac{\chi(1)^{2 m}}{q^{n^{2} \delta / 2}} .
\end{gathered}
$$

Now we set $\alpha^{*}:=7 / 8$ and choose $m \in \mathbb{Z}$ such that

$$
m k \leqslant \frac{n \delta}{4\left(1-\alpha^{*}\right)}=2 n \delta, \quad m k \leqslant \frac{40 n \alpha^{*}}{209}=\frac{35 n}{209}, \quad m k \geqslant \frac{11 n \delta}{10 \alpha^{*}}=\frac{44 n \delta}{35} .
$$

Note that $2 n \delta-44 n \delta / 35=26 n \delta / 35>5 n \delta / 8>k$. Furthermore, $2 n \delta \leqslant 35 n / 209$. Thus, there exists $m \in \mathbb{Z}$ satisfying (6.10). This choice of $m$ guarantees that

$$
\frac{19 m k^{2}}{8}+\frac{n^{2} \delta}{2 m} \leqslant \frac{5 k n \alpha^{*}}{11}+\frac{5 k n \alpha^{*}}{11}=\frac{10 k n \alpha^{*}}{11}
$$

Now we choose $\alpha=8 / 9$ and note by (6.5) that

$$
\frac{10 k n \alpha}{11}-\frac{10 k n \alpha^{*}}{11}=\frac{5 k n}{396} \geqslant \frac{420}{396}>1
$$

It follows from (6.11) that

$$
\frac{19 m k^{2}}{8}+\frac{n^{2} \delta}{2 m} \leqslant \frac{10 k n \alpha}{11}-1
$$

Using (6.9) and (6.6), we then get

$$
\left|\sum_{i=1}^{s} a_{i} \alpha_{i}(g)\right|^{1 / 2 m} \leqslant q^{\left(19 m^{2} k^{2} / 4+n^{2} \delta\right) / 2 m} \leqslant \chi(1)^{\alpha} / 2 .
$$

The choice (6.10) of $m$ also ensures that $k n\left(1-\alpha^{*}\right) \leqslant n^{2} \delta / 4 m$. Again,

$$
k n\left(1-\alpha^{*}\right)-k n(1-\alpha)=k n / 72>1
$$

by (6.5), whence $k n(1-\alpha) \leqslant n^{2} \delta / 4 m-1$. As $\chi(1) \leqslant q^{k n}$ by Theorem $1.2(\mathrm{i})$, using (6.9), we now obtain that

$$
\left|\sum_{j=1}^{t} b_{j} \beta_{j}(g)\right|^{1 / 2 m} \leqslant \frac{\chi(1)}{q^{n^{2} \delta / 4 m}} \leqslant \chi(1)^{\alpha} / 2 .
$$


Combining (6.8), (6.12), and (6.13) together and recalling $m \geqslant 1$, we arrive at

$$
\begin{aligned}
|\chi(g)| & \leqslant\left(\left|\sum_{i=1}^{s} a_{i} \alpha_{i}(g)\right|+\left|\sum_{j=1}^{t} b_{j} \beta_{j}(g)\right|\right)^{1 / 2 m} \\
& \leqslant\left|\sum_{i=1}^{s} a_{i} \alpha_{i}(g)\right|^{1 / 2 m}+\left|\sum_{j=1}^{t} b_{j} \beta_{j}(g)\right|^{1 / 2 m} \leqslant \chi(1)^{\alpha},
\end{aligned}
$$

as stated.

\section{Special linear and special unitary groups}

In this section, we extend the above results to special linear and special unitary groups.

Definition 2. For $n \in \mathbb{Z}_{\geqslant 1}$ and $\epsilon= \pm$, consider the subgroup $S:=\operatorname{SL}_{n}^{\epsilon}(q)$ of $G:=\mathrm{GL}_{n}^{\epsilon}(q)$. For any $\varphi \in \operatorname{Irr}(S)$, choose any $\chi \in \operatorname{Irr}(G)$ lying above $\varphi$. Then we define the level $\mathfrak{l}(\varphi)$ of $\varphi$ to be $\mathfrak{l}(\varphi):=\mathfrak{l}(\chi)$.

LEMMA 7.1. In the notation of Definition 2, the following statements hold:

(i) $\mathfrak{l}(\varphi)$ does not depend on the choice of $\chi \in \operatorname{Irr}(G)$ lying above $\varphi$.

(ii) $\mathfrak{l}(\varphi)$ is the smallest $j \in \mathbb{Z}_{\geqslant 0}$ such that $\varphi$ is an irreducible constituent of $\left.\left(\tau_{n}^{j}\right)\right|_{S}$ if $\epsilon=+$, respectively of $\left.\left(\zeta_{n}^{j}\right)\right|_{S}$ if $\epsilon=-$.

Proof. (i) Let $J$ denote the inertia subgroup of $\varphi$ in $G$. By the Clifford correspondence, $\chi=\operatorname{Ind}_{J}^{G}(\psi)$ for some $\psi \in \operatorname{Irr}(J)$ lying above $\varphi$. Since $J / S$ is cyclic, $\varphi$ extends to $J$ by [Is, Corollary (11.22)], whence any $\psi^{\prime} \in \operatorname{Irr}(J)$ lying above $\varphi$ is $\psi v$ for some $v \in \operatorname{Irr}(J / S)$ by Gallagher's theorem [Is, Corollary (6.17)]. Now if $\chi^{\prime} \in \operatorname{Irr}(G)$ is another character also lying above $\varphi$, then $\chi^{\prime}=\operatorname{Ind}_{J}^{G}\left(\psi^{\prime}\right)$ for some $\psi^{\prime}=\psi v \in \operatorname{Irr}(J / S)$ lying above $\varphi$. As $G / S$ is abelian, $\nu$ extends to some (linear) $\lambda \in \operatorname{Irr}(G / S)$. It follows that

$$
\chi^{\prime}=\operatorname{Ind}_{J}^{G}\left(\psi\left(\left.\lambda\right|_{S}\right)\right)=\operatorname{Ind}_{J}^{G}(\psi) \lambda=\chi \lambda,
$$

and so $\mathfrak{l}(\chi)=\mathfrak{l}\left(\chi^{\prime}\right)$ by Definition 1 .

(ii) Let $\tau$ be defined as in (1.1) so that $\tau=\tau_{n}$ if $\epsilon=+$ and $\tau=\zeta_{n}$ if $\epsilon=$ - , and let $\mathfrak{l}(\varphi)=j$. Then by Definitions 1 and 2, $\varphi$ is a constituent of $\left.\chi\right|_{s}=$ $\left.(\chi \lambda)\right|_{S}$ for some $\chi \in \operatorname{Irr}(G)$ and $\lambda \in \operatorname{Irr}(G / S)$, where $\chi \lambda$ is a constituent of $\tau^{j}$. Conversely, suppose that $\varphi$ is a constituent of $\left.\left(\tau^{k}\right)\right|_{S}$ for some $0 \leqslant k \leqslant j$. Then 
$\varphi$ is a constituent of $\left.\chi^{\prime}\right|_{S}$ for some $\chi^{\prime} \in \operatorname{Irr}(G)$ which is a constituent of $\tau^{k}$. It follows by Definition 2 and (i) that

$$
j=\mathfrak{l}(\varphi)=\mathfrak{l}\left(\chi^{\prime}\right) \leqslant k,
$$

and so $k=j$.

LEMMA 7.2. Let $G=\mathrm{GL}_{n}^{\epsilon}(q)$ and suppose that $\varphi \in \operatorname{Irr}\left(\operatorname{SL}_{n}^{\epsilon}(q)\right)$ does not extend to $G$. Then $\chi(1)>q^{n^{2} / 4-2}$ for any $\chi \in \operatorname{Irr}(G)$ lying above $\varphi$. In particular,

$$
\varphi(1)>q^{n^{2} / 4-2} /(q-\epsilon) \geqslant(2 / 3) q^{n^{2} / 4-3} .
$$

Proof. By hypothesis, $\chi \in \operatorname{Irr}(G)$ is reducible over $S$. Hence, the bound on $\chi(1)$ follows in the case $\epsilon=-$ from [LBST2, Theorem 3.9]. In the case $\epsilon=+$, this bound follows from [KT2, Proposition 5.10] and Proposition 5.9(i). Now the bound on $\varphi(1)$ also follows since $\chi(1) / \varphi(1) \leqslant[G: S]=q-\epsilon$.

Now we can prove the bounds for character degrees given in Theorem 1.3, which we restate below.

THEOREM 7.3. Let $n \geqslant 2, \epsilon= \pm$, and $S=\operatorname{SL}_{n}^{\epsilon}(q)$. Set $\sigma_{+}=1 /(q-1)$ and $\sigma_{-}=$ $1 / 2(q+1)$. Let $\varphi \in \operatorname{Irr}(S)$ have level $j=\mathfrak{l}(\varphi)$. Then the following statements hold.

(i) $\sigma_{\epsilon} q^{j(n-j)} \leqslant \varphi(1) \leqslant q^{n j}$.

(ii) If $j \geqslant n / 2$, then $\varphi(1)>q^{n^{2} / 4-2} /(q-\epsilon) \geqslant(2 / 3) q^{n^{2} / 4-3}$.

(iii) If $n \geqslant 7$ and $\left\lceil(1 / n) \log _{q} \varphi(1)\right\rceil<\sqrt{n-1}-1$, then

$$
\mathfrak{l}(\varphi)=\left\lceil\frac{\log _{q} \varphi(1)}{n}\right\rceil .
$$

Proof. Keep the notation of Definition 2. Since

$$
\chi(1) /(q-\epsilon) \leqslant \varphi(1) \leqslant \chi(1),
$$

(i) follows from Theorem 1.2(i). Next, (ii) follows from Theorem 1.2(ii) if $\varphi(1)=\chi(1)$ and from Lemma 7.2 otherwise. Certainly, (iii) also follows from Theorem 1.2(iii) if $\varphi(1)=\chi(1)$. Assume that $\varphi(1)<\chi(1)$. Then by Lemma 7.2, we have

$$
q^{n^{2} / 4-3.6}<\frac{q^{n^{2} / 4-2}}{q+1} \leqslant \frac{\chi(1)}{q+1} \leqslant \varphi(1),
$$


and so

$$
\left\lceil(1 / n) \log _{q} \varphi(1)\right\rceil \geqslant\lceil n / 4-3.6 / n\rceil>\sqrt{n-1}-1
$$

(as $n \geqslant 7$ ), a contradiction.

COROLlaRY 7.4. Theorems 1.4 and 1.5 hold for $\operatorname{SL}_{n}^{\epsilon}(q)$.

Proof. Let $S:=\operatorname{SL}_{n}^{\epsilon}(q)$. Certainly, the conclusions of Theorems 1.4 and 1.5 hold for any character $\varphi \in \operatorname{Irr}(S)$ that extend to $G:=\operatorname{GL}_{n}^{\epsilon}(q)$. If $\varphi$ is not extendable to $G$, then $\varphi(1)>q^{n^{2} / 4-3.6}$ by Lemma 7.2. We will again use the trivial bound $|\varphi(g)| \leqslant\left|\mathbf{C}_{S}(g)\right|^{1 / 2}$. For Theorem 1.4, taking $n \geqslant 2^{m+1} C+4$, we have $C n<$ $\left(n^{2} / 4-3.6\right) / 2^{m-1}$, and so

$$
|\varphi| \leqslant q^{C n / 2}<q^{\left(n^{2} / 4-3.6\right) / 2^{m}}<\varphi(1)^{1 / 2^{m}} .
$$

For Theorem 1.5, taking $n \geqslant 5$, we have

$$
|\varphi| \leqslant q^{n^{2} / 24}<q^{(8 / 9)\left(n^{2} / 4-3.6\right)}<\varphi(1)^{8 / 9} .
$$

Note that for $1 \leqslant n \leqslant 4$, there is no element $g \in S$ such that $\left|\mathbf{C}_{G}(g)\right| \leqslant q^{n^{2} / 12}$.

Now we are ready to prove Theorem 1.7 , which bounds the mixing time for various random walks on $\operatorname{SL}_{n}^{\epsilon}(q)$.

Proof of Theorem 1.7. We follow the proof of [BLST, Theorem 1.11]. Consider the Witten $\zeta$-function

$$
\zeta^{S}(s)=\sum_{\chi \in \operatorname{Irr}(S)} \frac{1}{\chi(1)^{s}} .
$$

By [LS, Theorem 1.1], $\lim _{q \rightarrow \infty} \zeta^{S}(s)=1$ as long as $s>2 / n$.

For (i), we have, by a well-known result (see [AH, Ch. 1, 10.1]) and Corollary 7.4, that

$$
\left\|P^{t}-U\right\|_{\infty} \leqslant \sum_{1_{S \neq \chi} \in \operatorname{Irr}(S)}\left(\frac{|\chi(g)|}{\chi(1)}\right)^{t} \chi(1)^{2} \leqslant \zeta^{S}(t / 9-2)-1 .
$$

Now, as $n \geqslant 19$, if $t \geqslant 19$, then $t / 9-2>2 / n$ and so the statement follows.

For (ii), note that $P^{t}(x)$ is the probability that a random walk on the Cayley graph $\Gamma(S, C)$ reaches $x$ after $t$ steps. Let

$$
\left\|P^{t}-U\right\|_{1}:=\sum_{x \in S}\left|P^{t}(x)-U(x)\right|
$$


By the Diaconis-Shahshahani bound [DS] and Theorem 1.5,

$$
\left(\left\|P^{t}-U\right\|_{1}\right)^{2} \leqslant \sum_{1_{S} \neq \chi \in \operatorname{Irr}(S)}\left(\frac{|\chi(g)|}{\chi(1)}\right)^{2 t} \chi(1)^{2} \leqslant \zeta^{S}(2 t / 9-2)-1 .
$$

As $n \geqslant 10$, if $t \geqslant 10$, then $2 t / 9-2>2 / n$, and the statement follows.

We conclude this section with another application. The Ore conjecture, now a theorem [LBST1], states that if $G$ is a finite nonabelian simple group, then the commutator map

$$
G \times G \rightarrow G, \quad(x, y) \mapsto x y x^{-1} y^{-1}
$$

is surjective, or, equivalently,

$$
\mu_{G}(g):=\sum_{\chi \in \operatorname{Irr}(G)} \frac{\chi(g)}{\chi(1)}>0
$$

for all $g \in G$. A strong qualitative refinement of the Ore conjecture was conjectured by Shalev [Sh, Conjecture 1.11] and states that if $G$ is a finite simple group of Lie type of bounded rank and $|G| \rightarrow \infty$, then the commutator map yields an almost uniform distribution on $G$; more precisely,

$$
\max _{1 \neq g \in G}\left|\mu_{G}(g)-1\right| \rightarrow 0 .
$$

However, this was disproved by Liebeck and Shalev (unpublished), by considering transvections in $\operatorname{SL}_{3}^{\epsilon}(q)$. A more recent conjecture of Avni and Shalev [ST, Conjecture 1.7] states that if $G$ is a simple group of Lie type of rank $r$, then

$$
\max _{1 \neq g \in G} \mu_{G}(g) \leqslant C(r)
$$

for some constant $C(r)$ possibly depending on $r$. We can now offer some evidence in support of this conjecture.

COROLlARY 7.5. For any $k \in \mathbb{Z}_{\geqslant 1}$, let $q_{k}$ be a prime power, $n_{k} \geqslant 19, \epsilon_{k}= \pm$, $G_{k}:=\mathrm{SL}_{n_{k}}^{\epsilon_{k}}\left(q_{k}\right)$, and let $g_{k} \in G_{k}$ be such that $\left|\mathbf{C}_{\mathrm{GL}_{n_{k}}^{\epsilon_{k}}\left(q_{k}\right)}\left(g_{k}\right)\right| \leqslant\left(q_{k}\right)^{n_{k}^{2} / 12}$. If $\mu_{G_{k}}$ is defined as in (7.2) and $\lim _{k \rightarrow \infty}\left|G_{k}\right|=\infty$, then

$$
\lim _{k \rightarrow \infty} \mu_{G_{k}}\left(g_{k}\right)=1 \text {. }
$$

Proof. By Theorem 1.5, $\left|\chi\left(g_{k}\right)\right| \leqslant \chi(1)^{8 / 9}$ for all $\chi \in \operatorname{Irr}\left(G_{k}\right)$, whence

$$
\left|\mu_{G_{k}}\left(g_{k}\right)-1\right| \leqslant \zeta^{G_{k}}(1 / 9)-1,
$$


where the zeta function $\zeta^{S}(s)$ is as defined in (7.1). Now the result follows by [LS, Theorem 1.1] (if $n_{k}$ is bounded) and [LS, Theorem 1.2] (if $n_{k}$ grows unbounded).

Very recently, it has been shown in [ST] that the upper bound $C(r)$ in the Avni-Shalev conjecture (7.3) must, in fact, depend on $r$, and similarly,

$$
\lim _{n \rightarrow \infty} \max _{1 \neq g \in \mathrm{A}_{n}} \mu_{\mathrm{A}_{n}}(g)=\infty .
$$

\section{Proofs of Theorems 1.1 and $\mathbf{1 . 6}$}

8.1. Dual pairs for $\mathbf{G L}_{\boldsymbol{n}}(\boldsymbol{q})$. In this subsection, we prove Theorems 1.1 and 1.6 for $G=\operatorname{GL}_{n}(q)$. We view $G=G_{n}=\operatorname{GL}(A)$, where $A=\mathbb{F}_{q}^{n}$, and consider $S=\mathrm{GL}(B) \cong \mathrm{GL}_{j}(q)$ with $B=\mathbb{F}_{q}^{j}$ and $1 \leqslant j \leqslant n$. Let $V:=A \otimes_{\mathbb{F}_{q}} B$, and consider the (reducible) Weil character $\tau$ of $\mathrm{GL}(V) \cong \mathrm{GL}_{n j}(q)$ as defined in (1.1). Note that

$$
\operatorname{dim}_{\mathbb{F}_{q}} \operatorname{Ker}\left(g-1_{V}\right)=j \cdot \operatorname{dim}_{\mathbb{F}_{q}} \operatorname{Ker}\left(g-1_{A}\right)
$$

for any $g \in G$. Hence, (1.1) and (3.1) imply that

$$
\left.\tau\right|_{G}=\left(\tau_{n}\right)^{j} .
$$

Any $v \in V=A \otimes_{\mathbb{F}_{q}} B$ can be written as $\sum_{i=1}^{t} a_{i} \otimes b_{i}$ for some $a_{i} \in A$, $b_{i} \in B$. Choosing such an expression with smallest possible $t$ for $v$, one then calls $t$ the rank of $v$; note that this rank cannot exceed $j=\operatorname{dim} B$. Note that $G$ acts transitively on the set $\Omega$ of all $v \in V$ of (largest possible) rank $j$; let $\rho$ denote the permutation character of $G \times S$ acting on $\Omega$.

Fix a basis $\left(e_{1}, \ldots, e_{n}\right)$ of $A$ and a basis $\left(f_{1}, \ldots, f_{j}\right)$ of $B$, and consider $v_{0}:=$ $\sum_{i=1}^{j} e_{i} \otimes f_{i}$. It is straightforward to check that $(g, s) \in \Gamma:=G \times S$ fixes $v_{0}$ exactly when

$$
g=\left(\begin{array}{cc}
{ }^{t} X^{-1} & * \\
0 & Y
\end{array}\right), \quad s=X, X \in \mathrm{GL}_{j}(q), Y \in \mathrm{GL}_{n-j}(q) .
$$

Denoting $R:=\operatorname{Stab}_{\Gamma}\left(v_{0}\right)$, we see that $\rho=\operatorname{Ind}_{R}^{\Gamma}\left(1_{R}\right)$. Consider the parabolic subgroup

$$
P=U \rtimes L:=\operatorname{Stab}_{G}\left(\left\langle e_{1}, \ldots, e_{j}\right\rangle_{\mathbb{F}_{q}}\right)
$$

of $G$, with its radical $U$ and Levi subgroup $L=\{\operatorname{diag}(X, Y)\}=\mathrm{GL}_{j} \times \mathrm{GL}_{n-j}$, where

$$
\mathrm{GL}_{j}:=\left\{\operatorname{diag}\left(X, I_{n-j}\right) \in L\right\}, \quad \mathrm{GL}_{n-j}:=\left\{\operatorname{diag}\left(I_{j}, Y\right) \in L\right\} .
$$


We can write $R=\tilde{U} \rtimes \tilde{L}$, where

$$
\begin{gathered}
\tilde{U}:=\left\{\left(u, I_{j}\right) \in \Gamma \mid u \in U\right\} \\
\tilde{L}=\left\{\left(\operatorname{diag}\left({ }^{t} X^{-1}, Y\right), X\right) \in \Gamma \mid X \in \mathrm{GL}_{j}(q), Y \in \mathrm{GL}_{n-j}(q)\right\} .
\end{gathered}
$$

Now consider any $\alpha \in \operatorname{Irr}(S)$ and $\delta \in \operatorname{Irr}(G)$, and express

$$
{ }^{*} R_{L}^{G}(\delta)=\gamma \otimes 1_{\mathrm{GL}_{n-j}}+\delta^{\prime},
$$

where $\gamma$ is a character of $\mathrm{GL}_{j}, \delta^{\prime}$ is a character of $L$ with no irreducible constituent having $\mathrm{GL}_{n-j}$ in its kernel, and ${ }^{*} R_{L}^{G}$ denotes the Harish-Chandra restriction (which is adjoint to the Harish-Chandra induction $R_{L}^{G}$ ). Also let $\sigma$ denote the transpose-inverse automorphism of $S: \sigma(X)={ }^{t} X^{-1}$. Note that $X \in S$ and ${ }^{t} X$ are $S$-conjugate. Hence,

$$
\gamma^{\sigma}(X)=\gamma(\sigma(X))=\gamma\left({ }^{t} X^{-1}\right)=\gamma\left(X^{-1}\right)=\bar{\gamma},
$$

that is, $\gamma^{\sigma}=\bar{\gamma}$. Hence, the value of $\left(\gamma \otimes 1_{\mathrm{GL}_{n-j}}\right) \otimes \alpha$ at a typical element $\left(\operatorname{diag}\left({ }^{t} X^{-1}, Y\right), X\right) \in \tilde{L}$ is $\gamma^{\sigma}(X) \alpha(X)=\bar{\gamma} \alpha(X)$.

Certainly, the kernel of $1_{R}$ contains $\tilde{U} \triangleleft R$ and also $\mathrm{GL}_{n-j}$. It then follows that

$$
\left[\left.(\delta \otimes \alpha)\right|_{R}, 1_{R}\right]_{R}=\left[\left.\left(\left(\gamma \otimes 1_{\mathrm{GL}_{n-j}}\right) \otimes \alpha\right)\right|_{\tilde{L}}, 1_{\tilde{L}}\right]_{\tilde{L}}=\left[\bar{\gamma} \alpha, 1_{\mathrm{GL}_{j}}\right]_{\mathrm{GL}_{j}}=[\gamma, \alpha]_{\mathrm{GL}_{j}} .
$$

Using (8.2) and the adjoint functor $R_{L}^{G}$, we also have

$$
[\gamma, \alpha]_{\mathrm{GL}_{j}}=\left[{ }^{*} R_{L}^{G}(\delta), \alpha \otimes 1_{\mathrm{GL}_{n-j}}\right]_{L}=\left[\delta, R_{L}^{G}\left(\alpha \otimes 1_{\mathrm{GL}_{n-j}}\right)\right]_{G} .
$$

Together with (8.4), this shows

$$
[\delta \otimes \alpha, \rho]_{\Gamma}=\left[\left.(\delta \otimes \alpha)\right|_{R}, 1_{R}\right]_{R}=\left[\delta, R_{L}^{G}\left(\alpha \otimes 1_{\mathrm{GL}_{n-j}}\right)\right]_{G} .
$$

Next we will use the proof of Proposition 3.8 applied to $\chi:=\alpha$, written in the form (3.12) with $s_{1}=1 \neq s_{2}, \ldots, s_{m}$. Also write

$$
\lambda_{1}=\left(\gamma_{2}, \ldots, \gamma_{r}\right) \vdash k, \quad \tilde{\lambda}_{1}:=\left(n-j, \gamma_{2}, \ldots, \gamma_{r}\right) .
$$

In combination with Theorem 3.9, the proof of Proposition 3.8 shows that all irreducible constituents of $R_{L}^{G}\left(\alpha \otimes 1_{\mathrm{GL}_{n-j}}\right)$ are of true level $\leqslant j$; moreover, if $\theta$ is such a constituent of true level $j$, then

$$
\theta=D_{\alpha}^{\circ}:=S\left(1, \tilde{\lambda}_{1}\right) \circ S\left(s_{2}, \lambda_{2}\right) \circ \cdots \circ S\left(s_{m}, \lambda_{m}\right),
$$


and $\theta$ occurs with multiplicity one. Note that $\tilde{\lambda}_{1}$ is a partition precisely when $n-j \geqslant \gamma_{2}$, which is equivalent to $\mathfrak{I}^{*}(\alpha)=j-\gamma_{2} \geqslant 2 j-n$. Writing the $\alpha$ isotypic component of $\rho$ as $E_{\alpha} \otimes \alpha$ and setting $D_{\alpha}^{\circ}:=0$ when $\mathfrak{l}^{*}(\alpha)<2 j-n$, we conclude from (8.5) that $E_{\alpha}-D_{\alpha}^{\circ}$ is a character of $G$ of true level smaller than $j$.

Recall that $G$ acts transitively on $\Omega$, with point stabilizer $R \cap G=U \rtimes$ $\mathrm{GL}_{n-j}=\operatorname{Stab}_{G}\left(e_{1}, \ldots, e_{j}\right)$. Hence, the proof of Proposition 3.2 implies that

$$
\left.\rho\right|_{G}=R_{L}^{G}\left(\operatorname{reg}_{\mathrm{GL}_{j}} \otimes 1_{\mathrm{GL}_{n-j}}\right) .
$$

Now, by Proposition 3.8, Theorem 3.9, and (8.1), $\left.(\tau-\rho)\right|_{G}=\left(\tau_{n}\right)^{j}-\left.\rho\right|_{G}$ has true level smaller than $j$. The same is true for all irreducible constituents of $F_{\alpha}$, where $F_{\alpha} \otimes \alpha$ is the $\alpha$-isotypic component of $\left.\tau\right|_{\Gamma}-\rho$. Since every irreducible character of $G$ of true level $j$ appears in $\left(\tau_{n}\right)^{j}$ by Definition 1 , such a character $\theta$ must be some $D_{\alpha}^{\circ}$ for some $\alpha \in \operatorname{Irr}(S)$ with $\mathfrak{l}^{*}(\alpha) \geqslant 2 j-n$, and (8.6) implies that

$$
\alpha=\left({ }^{*} R_{L}^{G}(\theta)\right)^{1_{\mathrm{GL}_{n-j}}} .
$$

Thus, we have completed the proof of statements (i), (ii) of Theorem 1.1 in the case $\epsilon=+$. We have also obtained an explicit formula for the bijection $\alpha \mapsto D_{\alpha}^{\circ}$ in Theorem 1.1(ii) in terms of Dipper-James parametrization.

COROLlaRy 8.1. Let $\mathfrak{l}^{*}(\alpha) \geqslant 2 j-n$ for $\alpha \in \operatorname{Irr}\left(\mathrm{GL}_{j}(q)\right)$ and express

$$
\alpha=S\left(1, \lambda_{1}\right) \circ S\left(s_{2}, \lambda_{2}\right) \circ \cdots \circ S\left(s_{m}, \lambda_{m}\right)
$$

as in (3.12), with $s_{i} \neq 1$ and $\lambda_{1}=\left(\gamma_{2}, \ldots, \gamma_{r}\right)$. Then in Theorem 1.1(ii), we have

$$
\Theta^{-1}(\alpha)=D_{\alpha}^{\circ}=S\left(1, \tilde{\lambda}_{1}\right) \circ S\left(s_{2}, \lambda_{2}\right) \circ \cdots \circ S\left(s_{m}, \lambda_{m}\right),
$$

with $\tilde{\lambda}_{1}=\left(n-j, \gamma_{2}, \ldots, \gamma_{r}\right)$.

Proof. This is just (8.6).

To prove statements (i) and (ii) of Theorem 1.6, we need some auxiliary statements. For any finite-dimensional vector space $U$ over a field $\mathbb{F}$ and any element $x \in \operatorname{GL}(U)$, let $d_{U}(x):=\operatorname{dim}_{\mathbb{F}} \operatorname{Ker}\left(x-1_{U}\right)$ and let $\delta_{U}(x)$ denote the largest dimension of $x$-eigenspaces on $U \otimes_{\mathbb{F}} \overline{\mathbb{F}}$.

Lemma 8.2. Let $\mathbb{F}$ be a field and let $V=A \otimes_{\mathbb{F}} B$ with $\operatorname{dim}_{\mathbb{F}} A=n$ and $\operatorname{dim}_{\mathbb{F}} B=$ $j$, and let $g \in \mathrm{GL}(A)$ with $\delta_{A}(g)=k$. Then the following statements hold.

(i) $d_{V}(g \otimes s) \leqslant k j$ for all $s \in \mathrm{GL}(B)$. 
(ii) If $k \geqslant n / 2$, then $d_{V}(g \otimes s) \leqslant k(j-2)+n$ for all but possibly one element $s \in \mathrm{GL}(B)$.

(iii) Assume $g \notin \mathbf{Z}(\mathrm{GL}(A))$ and $j \geqslant 2$. Then $d_{V}(g \otimes s) \leqslant(n-1)(j-1)+1$ for all but possibly one element $s \in \mathrm{GL}(B)$, and $d_{V}(g \otimes s) \leqslant(n-1) j$ for all $s \in \mathrm{GL}(B)$.

Proof. With no loss, we may replace $A, B, V$ by $A \otimes_{\mathbb{F}} \overline{\mathbb{F}}, B \otimes_{\mathbb{F}} \overline{\mathbb{F}}, V \otimes_{\mathbb{F}} \overline{\mathbb{F}}$, and, thus, assume that $\mathbb{F}=\overline{\mathbb{F}}$. Note that $d_{V}(h) \leqslant d_{U}(h)+d_{V / U}(h)$ for any $h \in \operatorname{GL}(V)$ and any $h$-invariant subspace $U \subseteq V$. Since $\mathbb{F}=\overline{\mathbb{F}}$, there exists a $g \otimes s$-invariant filtration of $V$ with all quotients isomorphic to $A$. Since the result is obvious for $j=1$, (i) follows.

For (ii), replacing $g$ by a scalar multiple (which does not change $\delta_{A}(g)$ ), we may assume that the 1-eigenspace of $g$ on $A$ has dimension $k$. First, suppose that $g$ is not unipotent. Write $A=A_{1} \oplus A_{2}$ with $A_{1}=\operatorname{Ker}\left((g-1)^{n}\right)$ and $A_{2}=$ $\operatorname{Im}\left((g-1)^{n}\right)$. Similarly, write $B=B_{1} \oplus B_{2}$ with $B_{1}=\operatorname{Ker}\left((s-1)^{j}\right)$ and $B_{2}=$ $\operatorname{Im}\left((s-1)^{j}\right)$. Applying (i) to $g \otimes s$ acting on $A_{1} \otimes B_{1}$, we get $d_{V}(g \otimes s) \leqslant$ $k\left(\operatorname{dim} B_{1}\right)+\left(\operatorname{dim} A_{2}\right)\left(\operatorname{dim} B_{2}\right)$, where the right-hand side is clearly maximized when $\operatorname{dim} A_{2}=n-k$, giving

$$
d_{V}(g \otimes s) \leqslant k\left(\operatorname{dim} B_{1}\right)+(n-k)\left(\operatorname{dim} B_{2}\right) .
$$

Since $k \geqslant n-k$, the right-hand side in the latter bound does not decrease as $\operatorname{dim} B_{1}$ grows. So if $B_{2} \neq 0$, this gives $d_{V}(g \otimes s) \leqslant k(j-2)+n$.

We will now prove the same inequality for $s \neq 1$ and $B_{2}=0$, that is, when $s$ is unipotent. Note that since $g \otimes s$ has no fixed point on $A_{2} \otimes B$, the result follows by induction on $n$ unless $A_{2}=0$, that is, $g$ is unipotent. Let $J_{i}$ denote the Jordan $i \times i$-block with eigenvalue 1 ; also use the symbol $m J_{i}$ to denote the direct sum of $m$ blocks $J_{i}$. By [S1, Theorem, page 685], if $a, b \in \mathbb{Z}_{\geqslant 1}$, then $J_{a} \otimes J_{b}$ is a direct sum of $\min (a, b)$ Jordan blocks, whence $d_{V}\left(J_{a} \otimes J_{b}\right)=\min (a, b)$. Applying the latter formula to various $(a, b)$, it follows for $h=J_{a} \oplus r J_{1}$ and $t=J_{b}$ with $r \geqslant a-2, a, b \geqslant 2$ that

$$
\begin{aligned}
d_{V}(h \otimes t) & =\min (a, b)+r \leqslant r+a+(r+1)(b-2) \\
& =d_{V}\left(h^{\sharp} \otimes t^{\mathrm{b}}\right) \leqslant(r+1) b=d_{V}\left(h^{\sharp} \otimes t^{0}\right),
\end{aligned}
$$

where we define

$$
h^{\sharp}:=(a-1) J_{2} \oplus(r+2-a) J_{1}, \quad t^{\mathrm{b}}:=J_{2} \oplus(b-2) J_{1}, \quad t^{0}:=b J_{1}
$$

for the given $h, t$. Write

$$
g=J_{a_{1}} \oplus J_{a_{2}} \oplus \cdots \oplus J_{a_{m}} \oplus v J_{1}, \quad s=J_{b_{1}} \oplus J_{b_{2}} \oplus \cdots \oplus J_{b_{l}}
$$


with $a_{1} \geqslant a_{2} \geqslant \cdots \geqslant a_{m} \geqslant 2, b_{1} \geqslant b_{2} \geqslant \cdots \geqslant b_{l} \geqslant 1$. Then the conditions $k \geqslant n / 2$ and $s \neq 1$ imply that $v \geqslant \sum_{i=1}^{m}\left(a_{i}-2\right)$ and $b_{1} \geqslant 2$. Thus, we can write $g=h_{1} \oplus \cdots \oplus h_{m}$ with $h_{i}=J_{a_{i}} \oplus r_{i} J_{1}$ and $r_{i} \geqslant a_{i}-2$. Applying (8.7), we see that $d_{V}(g \otimes s)$ does not decrease when we replace $g, s$ by

$$
g^{\sharp}:=h_{1}^{\sharp} \oplus h_{2}^{\sharp} \oplus \cdots \oplus h_{m}^{\sharp}, \quad s^{b}:=J_{b_{1}}^{b} \oplus J_{b_{2}}^{0} \oplus \cdots \oplus J_{b_{l}}^{0}=J_{2} \oplus(j-2) J_{1},
$$

which does not change $d_{A}(g)=k$. Thus,

$$
d_{V}(g \otimes s) \leqslant d_{V}\left(g^{\sharp} \otimes s^{\mathrm{b}}\right)=\sum_{i=1}^{m}\left(r_{i}+a_{i}\right)+\sum_{i=1}^{m}\left(r_{i}+1\right)\left(\sum_{i=1}^{l} b_{i}-2\right)=n+k(j-2) .
$$

For (iii), note that $g \notin \mathbf{Z}(\mathrm{GL}(A))$ implies that $k \leqslant n-1$, whence $d_{V}(g \otimes s) \leqslant$ $(n-1) j$ by (i). Furthermore, if $k<n / 2$, then by (i), we have

$$
d_{V}(g \otimes s)<n j / 2<(n-1)(j-1)+1
$$

as $j \geqslant 2$. If $k \geqslant n / 2$, then the statement follows from (ii).

Let $2 \leqslant j \leqslant n / 2$, and let $\chi \in \operatorname{Irr}(G)$ have $\mathfrak{l}(\chi)=j$. Multiplying $\chi$ by a suitable linear character, we may assume that $\mathfrak{l}^{*}(\chi)=j$. By Theorem 1.1(ii), $\chi=D_{\alpha}^{\circ}$ for some $\alpha \in \operatorname{Irr}(S)$. Consider any $g \in G \backslash \mathbf{Z}(G)$. We will now bound $D_{\alpha}^{\circ}(1)$ and $\left|D_{\alpha}^{\circ}(g)\right|$ using the well-known formula

$$
D_{\alpha}(g)=\frac{1}{|S|} \sum_{s \in S} \tau(g s) \bar{\alpha}(s) .
$$

According to Definition 1 and Theorem 1.1(ii), we can write

$$
\left.\tau\right|_{G}=\sum_{i=1}^{N} a_{i} \theta_{i}, \quad D_{\alpha}^{\prime}:=D_{\alpha}-D_{\alpha}^{\circ}=\sum_{i=1}^{N^{\prime}} b_{i} \theta_{i},
$$

where $\theta_{i} \in \operatorname{Irr}(G)$ are pairwise distinct, $a_{i}, b_{i} \in \mathbb{Z}_{\geqslant 0}, N \geqslant N^{\prime}, a_{i} \geqslant b_{i}$ if $i \leqslant N^{\prime}$, $\mathfrak{l}^{*}\left(\theta_{i}\right) \leqslant j$ for all $i$. In fact, if $i \leqslant N^{\prime}$, then $\mathfrak{l}^{*}\left(\theta_{i}\right) \leqslant j-1$, and so $\mathfrak{l}\left(\theta_{i}\right)=$ $\mathfrak{l}^{*}\left(\theta_{i}\right) \leqslant j-1<n / 2$ as $j \leqslant n / 2$, whence $\theta_{i}(1) \leqslant q^{n(j-1)}$ by Theorem 1.2. Let $k(X)=|\operatorname{Irr}(X)|$ denote the class number of a finite group $X$. By [FG1, Proposition 3.5], $k\left(\mathrm{GL}_{n}(q)\right) \leqslant q^{n}$. Note that $N^{\prime}$ cannot exceed the total number of irreducible characters of true level $<j$; hence, by Theorem 1.1(ii), we have

$$
N^{\prime} \leqslant \sum_{i=0}^{j-1} k\left(\mathrm{GL}_{i}(q)\right) \leqslant \sum_{i=0}^{j-1} q^{i}<q^{j}
$$


Also, $\sum_{i=1}^{N} a_{i}^{2}=\left[\left.\tau\right|_{G},\left.\tau\right|_{G}\right]_{G} \leqslant 8 q^{j^{2}}$ by Lemma 2.4. It follows that

$$
\left(\sum_{i=1}^{N^{\prime}} b_{i}\right)^{2} \leqslant N^{\prime} \sum_{i=1}^{N^{\prime}} b_{i}^{2} \leqslant q^{j} \sum_{i=1}^{N} a_{i}^{2} \leqslant 8 q^{j^{2}+j}
$$

and so

$$
D_{\alpha}^{\prime}(1) \leqslant \sum_{i=1}^{N^{\prime}} b_{i} q^{n(j-1)} \leqslant \sqrt{8 q^{j^{2}+j}} q^{n(j-1)} .
$$

Next, if $1 \neq s \in S$, then $|\tau(s)|=q^{n d_{B}(s)} \leqslant q^{n(j-1)}$, whence (8.8) implies that

$$
D_{\alpha}(1) \geqslant \alpha(1)\left(q^{n j}-|S| q^{n(j-1)}\right) /|S| .
$$

We may assume that $g \notin \mathbf{Z}(G)$ and so $k:=\delta_{A}(g) \leqslant n-1$. Then by Lemma 8.2(i), (iii), $|\tau(g s)|=q^{d_{V}(g s)} \leqslant q^{(n-1)(j-1)+1}$ for all but possibly one element $s \in S$, for which we have $|\tau(g s)| \leqslant q^{k j} \leqslant q^{(n-1) j}$. Hence, by (8.8), we have

$$
\left|D_{\alpha}(g)\right| \leqslant \alpha(1)\left(q^{k j}+|S| q^{(n-1)(j-1)+1}\right) /|S| .
$$

Since $\chi=D_{\alpha}^{\circ}=D_{\alpha}-D_{\alpha}^{\prime}$, we have $|\chi(g)| \leqslant\left|D_{\alpha}(g)\right|+D_{\alpha}^{\prime}(1)$. Using (8.10), we now obtain

$$
\begin{gathered}
\chi(1) \geqslant \alpha(1)\left(q^{n j}-|S| q^{n(j-1)}-|S| \sqrt{8 q^{j^{2}+j}} q^{n(j-1)}\right) /|S|, \\
|\chi(g)| \leqslant \alpha(1)\left(q^{k j}+|S| q^{(n-1)(j-1)+1}+|S| \sqrt{8 q^{j^{2}+j}} q^{n(j-1)}\right) /|S| .
\end{gathered}
$$

Now assume that $2 \leqslant j \leqslant \sqrt{(8 n-17) / 12}-1 / 2$. Then

$$
\begin{gathered}
(n-1)(j-1)+1 \leqslant n(j-1), \quad 1+\sqrt{8 q^{j^{2}+j}}<1.046 q^{j^{2}+j+3 / 2}, \\
3\left(j^{2}+j+1\right) / 2 \leqslant n-1,
\end{gathered}
$$

and so

$$
\begin{aligned}
q^{j^{2}+n(j-1)}\left(1+\sqrt{8 q^{j^{2}+j}}\right) & <1.046 q^{3\left(j^{2}+j+1\right) / 2+n(j-1)-j} \\
& \leqslant 1.046 q^{(n-1) j-1} \leqslant 0.523 q^{(n-1) j}
\end{aligned}
$$

As $S \mid<q^{j^{2}}$, it now follows from (8.11) that

$$
\chi(1) \geqslant \frac{q^{n j}\left(1-0.523 q^{-j}\right)}{|S| / \alpha(1)} \geqslant \frac{0.869 q^{n j}}{|S| / \alpha(1)}, \quad|\chi(g)| \leqslant \frac{1.523 q^{(n-1) j}}{|S| / \alpha(1)}
$$

and so $|\chi(g)|<1.76 \chi(1)^{1-1 / n}$. 
Next assume that $2 \leqslant j \leqslant(\sqrt{12 n-59}-1) / 6$. Then $3 j^{2}+j+3 \leqslant n-2$, and so

$$
\begin{aligned}
q^{j^{2}+n(j-1)}\left(1+\sqrt{8 q^{j^{2}+j}}\right) & <1.046 q^{\left(3 j^{2}+j+3\right) / 2+n(j-1)} \\
& \leqslant 1.046 q^{n(j-1 / 2)-1} \leqslant 0.523 q^{n(j-1 / 2)} .
\end{aligned}
$$

It now follows from (8.11) that

$$
\chi(1) \geqslant \frac{q^{n j}\left(1-0.523 q^{-j}\right)}{|S| / \alpha(1)} \geqslant \frac{0.869 q^{n j}}{|S| / \alpha(1)}, \quad|\chi(g)| \leqslant \frac{q^{k j}+0.523 q^{n(j-1 / 2)}}{|S| / \alpha(1)}
$$

and so

$$
|\chi(g)|<1.76 \chi(1)^{\max (1-1 / 2 j, k / n)},
$$

as stated in Theorem 1.6(i).

Since the case $j=0$ is obvious, it remains to consider the case $j=1$, whence $\chi$ is a Weil character (see Example 3.11). Suppose first that $j=1 \leqslant$ $\sqrt{(8 n-17) / 12}-1 / 2$, and so $n \geqslant 6$. It is easy to check that

$$
\chi(1) \geqslant\left(q^{n}-q\right) /(q-1), \quad|\chi(g)| \leqslant\left(q^{n-1}+q\right) /(q-1)
$$

and so again $|\chi(g)|<1.76 \chi(1)^{1-1 / n}$; in particular, (8.12) holds if $k=n-1$. We now consider the case $k \leqslant n-2$ and $j=1 \leqslant(\sqrt{12 n-59}-1) / 6$, that is, $n \geqslant 9$. If $q=2$, then

$$
\chi(1)=2^{n}-2, \quad|\chi(g)| \leqslant 2^{k}-2 \leqslant \chi(1)^{k / n} .
$$

If $k \leqslant(n-1) / 2$, then

$$
\chi(1)>q^{n-1}, \quad|\chi(g)| \leqslant q^{k} \leqslant \chi(1)^{1 / 2} .
$$

If $k \geqslant(n+1) / 2$, then

$\chi(1) \geqslant\left(q^{n}-q\right) /(q-1), \quad|\chi(g)|<\left(q^{k}+q^{n-k}+q\right) /(q-1)<1.76 \chi(1)^{k / n}$.

If $k=n / 2$ and $q \geqslant 3$, then

$$
\chi(1) \geqslant\left(q^{n}-q\right) /(q-1), \quad|\chi(g)|<\left(2 q^{k}+q\right) /(q-1)<1.76 \chi(1)^{1 / 2},
$$

completing the proof of (8.12) for $j=1$.

To prove Theorem 1.6(ii), note that if $\psi \in \operatorname{Irr}\left(\operatorname{SL}_{n}(q)\right)$ of level $j$ does not extend to $G$, then $j \geqslant 1$ (in particular, $n \geqslant 6$ as above) and any character $\chi \in$ $\operatorname{Irr}(G)$ lying above it has degree $\chi(1)>q^{n^{2} / 4-2}$ by Lemma 7.2. On the other hand, $\chi(1) \leqslant q^{n j}$ by Theorem 1.2 , a contradiction. Hence, $\psi$ extends to $G$, and the statement follows from Theorem 1.6(i).

Thus, we have completed the proof of Theorems 1.1 and 1.6 in the case $\epsilon=+$. 
8.2. The rank of $\mathbf{G U}_{\boldsymbol{n}}(\boldsymbol{q})$-characters. Let $m \geqslant 1, A=\mathbb{F}_{q^{2}}^{2 m}$ be endowed with a nondegenerate Hermitian form o with Gram matrix $\left(\begin{array}{cc}0 & I_{m} \\ I_{m} & 0\end{array}\right)$ in a basis $\left(u_{1}\right.$, $\left.\ldots, u_{m}, v_{1}, \ldots, v_{m}\right)$, where $I_{m}$ is the identity $m \times m$-matrix. Let $G=\mathrm{GU}(A) \cong$ $\mathrm{GU}_{2 m}(q)$, and consider the Siegel parabolic subgroup $P:=\operatorname{Stab}_{G}(U)$ of $G$, with unipotent radical $Q$, where $U:=\left\langle u_{1}, \ldots, u_{m}\right\rangle_{\mathbb{F}_{q^{2}}}$. Fix $\kappa \in \mathbb{F}_{q^{2}}$ with $\kappa^{q-1}=-1$, a nontrivial linear character $\psi:\left(\mathbb{F}_{q},+\right) \rightarrow \mathbb{C}^{\times}$, and let $\mathcal{H}_{m}:=\left\{X \in M_{m}\left(\mathbb{F}_{q^{2}}\right) \mid\right.$ $\left.X={ }^{t} X^{(q)}\right\}$. Then

$$
Q=\left\{[I, X]:=\left(\begin{array}{cc}
I_{m} & \kappa X \\
0 & I_{m}
\end{array}\right) \mid X \in \mathcal{H}_{m}\right\},
$$

and any linear character of $Q$ is of the form

$$
\lambda_{B}:\left(\begin{array}{cc}
I_{m} & \kappa X \\
0 & I_{m}
\end{array}\right) \mapsto \psi(\operatorname{Tr}(B X))
$$

for some $B \in \mathcal{H}_{m}$. The $\operatorname{rank} \mathrm{r}\left(\lambda_{B}\right)$ is now defined to be the rank of $B$.

Definition 3 [GH2, Section 4]. For any $\operatorname{GU}_{2 m}(q)$-character $\chi$, the $U$-rank of $\chi, r(\chi)$, is defined to be the largest among all the ranks $r(\lambda)$, where $\lambda$ is any irreducible constituent of $\left.\chi\right|_{Q}$ and $Q$ is the unipotent radical of the Siegel parabolic subgroup $P$.

(In fact, this definition can be extended to odd-dimensional unitary groups, but we will not need it here.)

As shown in the proof of [GMST, Lemma 12.5], all the Weil characters of $\mathrm{GU}_{2 m}(q)$ have rank 1 , that is, $\mathrm{r}\left(\zeta_{2 m}\right)=1$. Together with the subadditivity of matrix rank, this yields the following immediate consequence.

COROLlary 8.3. For any $\chi \in \operatorname{Irr}\left(\mathrm{GU}_{2 m}(q)\right), \mathrm{r}(\chi) \leqslant \mathfrak{l}(\chi) \leqslant \mathfrak{l}^{*}(\chi)$.

Next, fix an integer $1 \leqslant j \leqslant m$ and consider $B=\mathbb{F}_{q^{2}}^{j}$ endowed with a nondegenerate Hermitian form $\circ$ with an orthonormal basis $\left(e_{1}, \ldots, e_{j}\right)$. Then we can consider $V:=A \otimes_{\mathbb{F}_{q^{2}}} B$ with the Hermitian form defined via $(a \otimes b) \circ$ $\left(a^{\prime} \otimes b^{\prime}\right)=\left(a \circ a^{\prime}\right)\left(b \circ b^{\prime}\right)$ for all $a, a^{\prime} \in A$ and $b, b^{\prime} \in B$. We will also consider $V$ with a nondegenerate $\mathbb{F}_{q}$-bilinear symplectic form

$$
\left(w \mid w^{\prime}\right):=\operatorname{Tr}_{\mathbb{F}_{q^{2}} / \mathbb{F}_{q}}\left(\kappa w \circ w^{\prime}\right),
$$

with respect to which $U \otimes_{\mathbb{F}_{q^{2}}} B$ is a maximal totally isotropic subspace (of dimension $2 m j$ over $\mathbb{F}_{q}$ ). This gives rise to an embedding $\mathrm{GU}(V) \hookrightarrow \Gamma:=$ $\operatorname{Sp}(V) \cong \operatorname{Sp}_{4 m j}(q)$. 
PROPOSITION 8.4. Let $q$ be any odd prime power. Consider the dual pair $G \times$ $S \rightarrow \mathrm{GU}(V) \cong \mathrm{GU}_{2 m j}(q)$, where $G=\mathrm{GU}(A) \cong \mathrm{GU}_{2 m}(q)$ and $S=\mathrm{GU}(B) \cong$ $\mathrm{GU}_{j}(q)$, with $1 \leqslant j \leqslant m$. Also consider the Siegel parabolic subgroup $P=$ $\operatorname{Stab}_{G}\left(\left\langle u_{1}, \ldots, u_{m}\right\rangle_{\mathbb{F}_{q^{2}}}\right)$ and its radical $Q$. Let $\lambda \in \operatorname{Irr}(Q)$ be any character of rank $j$, and let $\tau$ be the reducible Weil character of $\mathrm{GU}(V)$ as given in (1.1). Then the restriction of $\left.\tau\right|_{G \times S}$ to $Q \times S$ contains the character $\lambda \otimes \mathbf{r e g}_{S}$.

Proof. Consider a pair of complementary maximal totally isotropic $\mathbb{F}_{q^{-}}$ subspaces in the $\mathbb{F}_{q}$-space $V$, with complementary Witt bases:

$$
W:=\left\langle u_{i} \otimes e_{j}, \kappa u_{i} \otimes e_{j}\right\rangle_{\mathbb{F}_{q}}, \quad W^{\prime}:=\left\langle\frac{1}{2 \kappa} v_{i} \otimes e_{j}, \frac{1}{2 \kappa^{2}} v_{i} \otimes e_{j}\right\rangle_{\mathbb{F}_{q}} .
$$

Let $\chi_{2}$ denote the unique linear character of order 2 of $\mathrm{GU}(V)$, and let $\varpi$ denote a reducible Weil character of degree $q^{2 m j}$, as constructed in [Gr, Section 13]. Then it is known that $\left.\varpi\right|_{\mathrm{GU}(V)}=\chi_{2} \tau$; see [TZ, Section 4]. We will use the model given in [Gr, Section 13] for a representation affording the character $\varpi$, with $\Gamma$ acting on the space $\mathcal{W}$ of complex-valued functions on $W^{\prime}$. If $\delta_{u}$ denotes the delta function for any point $u \in W^{\prime}$, then $\left(\delta_{u} \mid u \in W^{\prime}\right)$ is a basis for $\mathcal{W}$. The action of the Siegel parabolic subgroup $\operatorname{Stab}_{\Gamma}(W)$ in this basis is described in [Gr, (13.3)].

Now fix the following vector:

$$
w:=4\left(v_{1} \otimes e_{1}+v_{2} \otimes e_{2}+\cdots+v_{j} \otimes e_{j}\right) .
$$

First, we consider the action of any $g=[I, X] \in Q$, given in the form of (8.13). Using [Gr, (13.3)], we have

$$
g\left(\delta_{v_{1} \otimes e_{1}}\right)=\psi\left(\frac{1}{2}\left((g-1)\left(v_{1} \otimes e_{1}\right) \mid v_{1} \otimes e_{1}\right)\right)=\psi\left(x_{11}\right) \delta_{v_{1} \otimes e_{1}},
$$

where we recall that $X=\left(x_{k l}\right) \in \mathcal{H}_{m}$. The same computation shows that

$$
g\left(\delta_{w}\right)=\psi\left(x_{11}+x_{22}+\cdots+x_{j, j}\right) \delta_{w} .
$$

Choosing

$$
Y:=\operatorname{diag}(\underbrace{1,1, \ldots, 1}_{j \text { times }}, \underbrace{0,0, \ldots, 0}_{m-j \text { times }})
$$

we then have

$$
g\left(\delta_{w}\right)=\psi(\operatorname{tr}(X Y)) \delta_{w}=\lambda_{Y}(g) \delta_{w},
$$

for all $g \in Q$, cf. (8.14), and note that $r\left(\lambda_{Y}\right)=j$. 
On the other hand, if $s \in S=\mathrm{GU}(B)$, then $s$ fixes both $W$ and $W^{\prime}$, and, moreover, $s(w)=w$ if and only if $s=1$. As the action of $Q \times S$ on $\mathcal{W}$ is monomial in the basis $\left(\delta_{u} \mid u \in W^{\prime}\right)$, we conclude that

$$
\mathcal{W}^{\prime}:=\left\langle\delta_{s(w)} \mid s \in S\right\rangle_{\mathbb{C}}
$$

is a $Q \times S$-module, with character $\operatorname{Ind}_{Q}^{Q \times S}\left(\lambda_{Y} \otimes 1_{S}\right)=\lambda_{Y} \otimes \operatorname{reg}_{S}$. Thus, the restriction of $\varpi$ to $Q \times S$ contains $\lambda_{Y} \otimes \mathbf{r e g}_{S}$, and the same is also true for $\tau=\chi_{2}\left(\left.\varpi\right|_{\mathrm{GU}(V)}\right)$. Since $P$ acts transitively on the set of $Q$-characters of any given rank, the statement follows.

COROLlaRY 8.5. In the notation of Theorem 1.1 , assume that $\epsilon=-, 1 \leqslant j \leqslant$ $n / 2$ and that $q$ is odd. Then for any $\alpha \in \operatorname{Irr}\left(\mathrm{GU}_{j}(q)\right), \mathfrak{l}^{*}\left(D_{\alpha}\right)=j$.

Proof. First, note that $\mathfrak{l}^{*}\left(D_{\alpha}\right) \leqslant \mathfrak{l}^{*}\left(\left(\zeta_{n}\right)^{j}\right)=j$. Assume that $\mathfrak{l}^{*}\left(D_{\alpha}\right)<j$ for some $\alpha \in \operatorname{Irr}(S)$.

(i) Consider the case $n=2 m$. By Proposition 8.4, $\left.\tau\right|_{Q \times S}$ contains $\lambda \otimes \mathbf{r e g}_{S}$ for some $\lambda \in \operatorname{Irr}(Q)$ of rank $j$. It follows that $\left.\left(D_{\alpha} \otimes \alpha\right)\right|_{Q \times S}$ contains $\lambda \otimes \alpha$, and so $\left.\left(D_{\alpha}\right)\right|_{Q}$ contains $\lambda$. The latter means that $\mathrm{r}\left(D_{\alpha}\right) \geqslant j$, and so $\mathfrak{l}^{*}\left(D_{\alpha}\right) \geqslant j$ by Corollary 8.3, contrary to the assumption.

(ii) Now let $n=2 m+1$ and embed $G_{2 m} \times G_{1}=\operatorname{GU}_{2 m}(q) \times \mathrm{GU}_{1}(q)$ in $G=$ $\mathrm{GU}_{n}(q)$. We can consider the dual pair $G_{2 m} \times S \rightarrow \mathrm{GU}_{2 m j}(q)$ and decompose

$$
\left.\zeta_{2 m j}\right|_{G_{2 m} \times S}=\sum_{\beta \in \operatorname{Irr}(S)} E_{\beta} \otimes \beta
$$

where $E_{\beta}$ is a $G_{2 m}$-character (possibly zero). Then we have

$$
\left.\sum_{\beta \in \operatorname{Irr}(S)}\left(D_{\beta}\right)\right|_{G_{2 m}} \otimes \beta=\left.\tau\right|_{G_{2 m} \times S}=\left(\sum_{\beta \in \operatorname{Irr}(S)} E_{\beta} \otimes \beta\right)\left(1_{G_{2 m}} \otimes \zeta_{j}\right) .
$$

Comparing the $\alpha$-isotypic components, we obtain

$$
\left.\left(D_{\alpha}\right)\right|_{G_{2 m}}=\sum_{\beta \in \operatorname{Irr}(S)}\left(\beta \zeta_{j}, \alpha\right)_{G_{2 m}} E_{\beta} .
$$

By definition, the assumption $\mathfrak{I}^{*}\left(D_{\alpha}\right)<j$ implies that there is some $0 \leqslant k<$ $j$ such that all irreducible constituents of $D_{\alpha}$ are contained in $\left(\zeta_{n}\right)^{k}$. Since $\left.\left(\zeta_{n}\right)\right|_{G_{2 m}}=q \zeta_{2 m}($ see [TZ, Lemma 4.2]), it follows that

$$
\mathfrak{l}^{*}\left(\left.\left(D_{\alpha}\right)\right|_{G_{2 m}}\right) \leqslant k<j
$$


On the other hand, we can choose an irreducible constituent $\beta \in \operatorname{Irr}(S)$ of $\alpha \overline{\zeta_{j}}$. Then

$$
1 \leqslant\left(\beta, \alpha \overline{\zeta_{j}}\right)_{G_{2 m}}=\left(\beta \zeta_{j}, \alpha\right)_{G_{2 m}},
$$

and so $\left(D_{\alpha}\right)_{G_{2 m}}$ contains $E_{\beta}$ by $(8.15)$. Now $\mathfrak{l}^{*}\left(E_{\beta}\right)=j$ by (i), and this contradicts (8.16).

8.3. Dual pairs for $\mathbf{G U}_{\boldsymbol{n}}(\boldsymbol{q})$. In this subsection, we prove Theorems 1.1 and 1.6 for $G=\operatorname{GU}_{n}(q)$. We view $G=G_{n}=\mathrm{GU}(A)$, where $A=\mathbb{F}_{q^{2}}^{n}$, and consider $S=\mathrm{GU}(B) \cong \mathrm{GU}_{j}(q)$ with $B=\mathbb{F}_{q^{2}}^{j}$ and $1 \leqslant j \leqslant n$. Let $V:=A \otimes_{\mathbb{F}_{q^{2}}} B$, and consider the (reducible) Weil character $\tau$ of $\mathrm{GU}(V) \cong \mathrm{GU}_{n j}(q)$ as defined in (1.1). Also keep the notation $G_{i}=\mathrm{GU}_{i}(q)$ for $1 \leqslant i \leqslant n$. Note that statement (i) of Theorem 1.1 is now just part of Theorem 3.9(i).

Suppose now that $2 \leqslant j \leqslant n / 2$ and consider any $\alpha \in \operatorname{Irr}\left(G_{j}\right)$. Let $D_{\alpha}^{\prime}$ denote the sum of all irreducible constituents of true level $<j$ of $D_{\alpha}$, counting with their multiplicities so that $D_{\alpha}^{\circ}:=D_{\alpha}-D_{\alpha}^{\prime}$ is a character, all of whose irreducible constituents have true level $j$. If, in addition, $q$ is odd, then $D_{\alpha}^{\circ}$ is nonzero by Corollary 8.5.

We will again use (8.8) and express $\left.\tau\right|_{G}$ and $D_{\alpha}^{\prime}$ as in (8.9). In particular, $\theta_{i} \in$ $\operatorname{Irr}(G)$ are pairwise distinct, $a_{i}, b_{i} \in \mathbb{Z}_{\geqslant 0}, N \geqslant N^{\prime}, a_{i} \geqslant b_{i}$ if $i \leqslant N^{\prime}, \mathfrak{I}^{*}\left(\theta_{i}\right) \leqslant j$ for all $i$. In fact, if $i \leqslant N^{\prime}$, then $\mathfrak{l}^{*}\left(\theta_{i}\right) \leqslant j-2$ by Corollary 4.8, and so $\mathfrak{l}\left(\theta_{i}\right)=$ $\mathfrak{l}^{*}\left(\theta_{i}\right) \leqslant j-2<n / 2$ as $j \leqslant n / 2$, whence $\theta_{i}(1) \leqslant q^{n(j-2)}$ by Theorem 1.2. According to [FG1, Section 3.3], $k\left(\mathrm{GU}_{n}(q)\right) \leqslant 8.26 q^{n}$. Note that $N^{\prime}$ cannot exceed the total number of irreducible characters of true level $\leqslant j-2$; hence, by Theorem 1.1(i), we have

$$
N^{\prime} \leqslant \sum_{i=0}^{j-2} k\left(\mathrm{GU}_{i}(q)\right) \leqslant 8.26 \sum_{i=0}^{j-2} q^{i}<8.26 q^{j-1} .
$$

Also, $\sum_{i=1}^{N} a_{i}^{2}=\left[\left.\tau\right|_{G},\left.\tau\right|_{G}\right]_{G}=\left[\left(\zeta_{n}\right)^{2 j}, 1_{G}\right]_{G} \leqslant 2 q^{j^{2}}$ by Lemma 2.4. It follows that

$$
\left(\sum_{i=1}^{N^{\prime}} b_{i}\right)^{2} \leqslant N^{\prime} \sum_{i=1}^{N^{\prime}} b_{i}^{2} \leqslant 8.26 q^{j-1} \sum_{i=1}^{N} a_{i}^{2} \leqslant 16.52 q^{j^{2}+j-1}
$$

and so

$$
D_{\alpha}^{\prime}(1) \leqslant \sum_{i=1}^{N^{\prime}} b_{i} q^{n(j-2)} \leqslant \sqrt{16.52 q^{j^{2}+j-1}} q^{n(j-2)} .
$$

As before, if $1 \neq s \in S$, then $|\tau(s)|=q^{n d_{B}(s)} \leqslant q^{n(j-1)}$, whence (8.8) implies that

$$
D_{\alpha}(1) \geqslant \alpha(1)\left(q^{n j}-|S| q^{n(j-1)}\right) /|S| \text {. }
$$


We may assume that $g \notin \mathbf{Z}(G)$ and so $k:=\delta_{A}(g) \leqslant n-1$. Then by Lemma 8.2(i), (iii), $|\tau(g s)|=q^{d_{V}(g s)} \leqslant q^{(n-1)(j-1)+1}$ for all but possibly one element $s \in S$, for which we have $|\tau(g s)| \leqslant q^{k j} \leqslant q^{(n-1) j}$. Hence, by (8.8), we have

$$
\left|D_{\alpha}(g)\right| \leqslant \alpha(1)\left(q^{k j}+|S| q^{(n-1)(j-1)+1}\right) /|S| .
$$

Using (8.17) and the estimate $\left|D_{\alpha}^{\circ}(g)\right| \leqslant\left|D_{\alpha}(g)\right|+D_{\alpha}^{\prime}(1)$, we obtain

$$
\begin{gathered}
D_{\alpha}^{\circ}(1) \geqslant \alpha(1)\left(q^{n j}-|S| q^{n(j-1)}-|S| \sqrt{16.52 q^{j^{2}+j-1}} q^{n(j-2)}\right) /|S|, \\
\left|D_{\alpha}^{\circ}(g)\right| \leqslant \alpha(1)\left(q^{k j}+|S| q^{(n-1)(j-1)+1}+|S| \sqrt{16.52 q^{j^{2}+j-1}} q^{n(j-2)}\right) /|S| .
\end{gathered}
$$

Note that $|S| \leqslant 1.5 q^{j^{2}}$ by Lemma 5.1(iii).

Now assume that $2 \leqslant j \leqslant \sqrt{n-3 / 4}-1 / 2$; in particular, $n \geqslant 7$. Then $j^{2}+j \leqslant$ $n-1,(n-1)(j-1)+1 \leqslant n(j-1)$, and so

$$
\begin{aligned}
& 1.5 q^{j^{2}+n(j-1)}\left(1+\sqrt{16.52 q^{j^{2}+j-1-2 n}}\right)<1.77 q^{j^{2}+n(j-1)} \\
& \leqslant 1.77 q^{(n-1) j-1} \leqslant 0.885 q^{(n-1) j} .
\end{aligned}
$$

It now follows from (8.18) that

$$
D_{\alpha}^{\circ}(1) \geqslant \frac{q^{n j}\left(1-0.885 q^{-j}\right)}{|S| / \alpha(1)} \geqslant \frac{0.778 q^{n j}}{|S| / \alpha(1)}, \quad\left|D_{\alpha}^{\circ}(g)\right| \leqslant \frac{1.885 q^{(n-1) j}}{|S| / \alpha(1)}
$$

and so $D_{\alpha}^{\circ}$ is a nonzero character of $G$ and $\left|D_{\alpha}^{\circ}(g)\right|<2.43 D_{\alpha}^{\circ}(1)^{1-1 / n}$.

Next assume that $2 \leqslant j \leqslant \sqrt{n / 2-1}$; in particular, $n \geqslant 10$. Then $j^{2} \leqslant n / 2-1$, and so

$$
\begin{aligned}
& 1.5 q^{j^{2}+n(j-1)}\left(1+\sqrt{16.52 q^{j^{2}+j-1-2 n}}\right)<1.77 q^{j^{2}+n(j-1)} \\
& \leqslant 1.77 q^{n(j-1 / 2)-1} \leqslant 0.885 q^{n(j-1 / 2)} .
\end{aligned}
$$

It now follows from (8.18) that

$$
D_{\alpha}^{\circ}(1) \geqslant \frac{q^{n j}\left(1-0.885 q^{-j}\right)}{|S| / \alpha(1)} \geqslant \frac{0.778 q^{n j}}{|S| / \alpha(1)}, \quad\left|D_{\alpha}^{\circ}(g)\right| \leqslant \frac{q^{k j}+0.885 q^{n(j-1 / 2)}}{|S| / \alpha(1)}
$$

and so $\left|D_{\alpha}^{\circ}(g)\right|<2.43 D_{\alpha}^{\circ}(1)^{\max (1-1 / 2 j, k / n)}$.

We have shown that, for any $\alpha \in \operatorname{Irr}(S), D_{\alpha}^{\circ}$ is a nonzero $G$-character that involves only characters of true level $j$. As $\left.\tau\right|_{G}=\sum_{\alpha \in \operatorname{Irr}(S)} \alpha(1)\left(D_{\alpha}^{\circ}+D_{\alpha}^{\prime}\right)$, it follows that the total sum $\Sigma$ of all multiplicities of irreducible constituents of true 
level $j$ in $\left.\tau\right|_{G}$ is at least $\sum_{\alpha \in \operatorname{Irr}(S)} \alpha(1)$. On the other hand, $\Sigma=\sum_{\alpha \in \operatorname{Irr}(S)} \alpha(1)$ by Theorem 3.9(i), and, furthermore, each character of true level $j$ enters some $D_{\alpha}^{\circ}$, and the total number of such characters is $k(S)$. We conclude that the characters $D_{\alpha}^{\circ}$ are all irreducible, pairwise distinct, and account for all characters of true level $j$ of $G$.

Suppose now $\chi \in \operatorname{Irr}(G)$ have $\mathfrak{l}(\chi)=j$ and $2 \leqslant j \leqslant \sqrt{n-3 / 4}-1 / 2$ as above. Multiplying $\chi$ by a suitable linear character, we may assume that $\mathrm{I}^{*}(\chi)=j$. By what we have just shown, $\chi=D_{\alpha}^{\circ}$ for some $\alpha \in \operatorname{Irr}(S)$. We have, therefore, proved Theorems 1.1(iii) and 1.6(iii) for $j \geqslant 2$.

Since the case $j=0$ is obvious, it remains to prove Theorems 1.1(iii) and 1.6(iii) for $j=1$. In this case, statement 1.1(iii) is well known, and $D_{\alpha}=D_{\alpha}^{\circ}$; furthermore, $\chi$ is a Weil character (see Example 3.12). First, suppose that $j=1 \leqslant \sqrt{n-3 / 4}-1 / 2$, and so $n \geqslant 4$. It is easy to check that

$$
\chi(1) \geqslant\left(q^{n}-q\right) /(q+1), \quad|\chi(g)| \leqslant\left(q^{n-1}+q\right) /(q+1)
$$

and so again $|\chi(g)|<2.43 \chi(1)^{1-1 / n}$. Assume now that $j=1 \leqslant \sqrt{(n-2) / 2}$, that is, $n \geqslant 6$. If $k \leqslant(n-1) / 2$, then

$$
\chi(1)>\left(q^{n}-q\right) /(q+1), \quad|\chi(g)| \leqslant q^{k}<2.43 \chi(1)^{1 / 2} .
$$

If $k \geqslant n / 2$, then

$$
\chi(1) \geqslant\left(q^{n}-q\right) /(q+1), \quad|\chi(g)|<\left(2 q^{k}+q\right) /(q+1)<2.43 \chi(1)^{k / n},
$$

completing the proof of Theorem 1.6(iii) for $j=1$.

Theorem 1.6(iv) can now be proved by exactly the same argument as we had in the proof of Theorem 1.6(ii). Thus, we have completed the proof of Theorems 1.1 and 1.6. Note that Theorem 1.1(iii) is weaker than Theorem 1.1(ii). The reason is that in the case $\epsilon=-$, we do not have an explicit geometric model for the reducible Weil representation of $\mathrm{GL}^{\epsilon}(V)=\mathrm{GL}_{n j}^{\epsilon}(q)$ (affording the character $\tau$ defined in (1.1)), which would allow us to have better control on $D_{\alpha} \otimes \alpha$ for any $\alpha \in \operatorname{Irr}\left(\mathrm{GL}_{j}^{\epsilon}(q)\right)$, as we did in Section 8.1.

COROLlaRY 8.6. Let $G=\mathrm{GL}_{n}^{\epsilon}(q) \geqslant S=\mathrm{SL}_{n}^{\epsilon}(q)$ with $\epsilon= \pm$, and let $0 \leqslant j<$ $n / 2$. Then the following statements hold:

(i) If $\chi \in \operatorname{Irr}(G)$ has $\mathfrak{l}(\chi)=j$, then $\varphi:=\left.\chi\right|_{S}$ is irreducible. Furthermore,

$$
\operatorname{Irr}(G \mid \varphi)=\{\chi \lambda \mid \lambda \in \operatorname{Irr}(G / S)\}
$$

and it contains a unique character of true level $j$. 
(ii) If $\Theta$ is the bijection in Theorem 1.1(i), then $\Lambda:\left.\alpha \mapsto\left(\Theta^{-1}(\alpha)\right)\right|_{S}$ is a bijection between $\operatorname{Irr}\left(\mathrm{GL}_{j}^{\epsilon}(q)\right)$ and $\{\varphi \in \operatorname{Irr}(S) \mid \mathfrak{l}(\varphi)=j\}$.

Proof. (i) Replacing $\chi$ by $\chi \lambda$ for a suitable $\lambda \in \operatorname{Irr}(G / S)$, we may assume that $\mathfrak{l}^{*}(\chi)=j$. By Theorem 3.9, the first part of the partition $\gamma_{1}$ is $n-j$. It follows that for any $\delta \in \mu_{q-\epsilon} \backslash\{1\}$, the first part of $\gamma_{\delta}$ is $\leqslant j$, whence $\mathfrak{l}^{*}(\chi \beta) \geqslant n-j>j$ and so $\chi \beta \neq \chi$ for any $1_{G} \neq \beta \in \operatorname{Irr}(G / S)$. As $G / S$ is cyclic, the statements now follow from [KT1, Lemma 3.2] and Gallagher's theorem [Is, (6.17)].

(ii) For $\alpha \in \operatorname{Irr}\left(\operatorname{GL}_{j}^{\epsilon}(q)\right)$, let $\chi:=\Theta^{-1}(\alpha)$ and $\varphi:=\left.\chi\right|_{S}=\Lambda(\alpha)$. Then $\mathfrak{l}^{*}(\chi)=$ $j<n / 2$, and the same arguments as in (i) show that $\mathfrak{l}(\chi)=j$. Now $\varphi \in \operatorname{Irr}(S)$ by (i) and $\mathfrak{l}(\varphi)=\mathfrak{l}(\chi)=j$ by Definition 2. Suppose now that $\varphi=\Lambda\left(\alpha^{\prime}\right)$ for some $\alpha^{\prime} \in \operatorname{Irr}\left(\mathrm{GL}_{j}^{\epsilon}(q)\right)$. Then $\left.\left(\chi^{\prime}\right)\right|_{S}=\varphi=\left.\chi\right|_{S}$ for $\chi^{\prime}:=\Theta^{-1}\left(\alpha^{\prime}\right)$. By (i), $\chi^{\prime}=\chi \beta$ for some $\beta \in \operatorname{Irr}(G / S)$. Since $\mathfrak{l}^{*}\left(\chi^{\prime}\right)=j=\mathfrak{l}^{*}(\chi)$, the arguments in (i) show that $\beta=1_{G}$ and $\chi^{\prime}=\chi$, whence $\alpha^{\prime}=\alpha$. Thus, $\Lambda$ is injective. Finally, suppose $\varphi_{1} \in \operatorname{Irr}(S)$ has $\mathfrak{l}\left(\varphi_{1}\right)=j$. Then $\mathfrak{l}\left(\chi_{1}\right)=j$ for some $\chi_{1} \in \operatorname{Irr}\left(G \mid \varphi_{1}\right)$ again by Definition 2. Replacing $\chi_{1}$ by $\chi_{1} \delta$ for a suitable $\delta \in \operatorname{Irr}(G / S)$, we may assume that $\mathfrak{I}^{*}\left(\chi_{1}\right)=j$. Now $\varphi_{1}=\left.\left(\chi_{1}\right)\right|_{S}$ by (i) and so $\varphi_{1}=\Lambda\left(\Theta\left(\chi_{1}\right)\right)$, proving the surjectivity of $\Lambda$.

\section{Some further results}

9.1. The level and the rank of $\mathbf{G L}_{\boldsymbol{n}}(q)$-characters. Again consider $G=$ $\mathrm{GL}(A) \cong \mathrm{GL}_{n}(q)$ with $q$ a power of a prime $p$ and $A=\left\langle e_{1}, \ldots, e_{n}\right\rangle_{\mathbb{F}_{q}}$, and fix a primitive complex $p$ th root $\varepsilon$ of unity. Consider the subspace $W_{j}=\left\langle e_{1}\right.$, $\left.\ldots, e_{j}\right\rangle_{\mathbb{F}_{q}}$ for any $1 \leqslant j \leqslant n / 2$ and its stabilizer $P_{j}=U_{j} \rtimes L_{j}$ with unipotent radical

$$
U_{j}=\left\{\left[I_{j}, X\right]:=\left(\begin{array}{cc}
I_{j} & X \\
0 & I_{n-j}
\end{array}\right) \mid X \in M_{j, n-j}\left(\mathbb{F}_{q}\right)\right\} .
$$

Note that the $\mathbb{F}_{q}$-bilinear form $(X, Y) \mapsto \operatorname{tr}\left(X \cdot{ }^{t} Y\right)$ is nondegenerate on $M_{j, n-j}\left(\mathbb{F}_{q}\right)$. It follows that any character $\lambda \in \operatorname{Irr}\left(U_{j}\right)$ can be written uniquely in the form

$$
\lambda=\lambda_{Y}:\left[I_{j}, X\right] \mapsto \varepsilon^{\operatorname{Tr}_{\mathbb{F}_{q} / \mathbb{F}_{p}} \operatorname{tr}\left(X \cdot{ }^{t} Y\right)}
$$

for some $Y \in M_{j, n-j}\left(\mathbb{F}_{q}\right)$, and the rank of $\lambda_{Y}$ is defined to $\operatorname{be} r\left(\lambda_{Y}\right):=\operatorname{rank}(Y)$.

Definition 4 [GH2, Definition 4.2.1]. For any $\mathrm{GL}_{n}(q)$-character $\chi$, the $U$ rank of $\chi, r(\chi)$, is defined to be the largest among all the ranks $r(\lambda)$, where $\lambda$ is any irreducible constituent of $\left.\chi\right|_{U_{j}}$ and $1 \leqslant j \leqslant n / 2$.

As we will see from Theorem 9.5, the $U$-rank $r(\chi)$ is related to, but coarser than, the level $\mathfrak{l}(\chi)$. First, we record some elementary properties of $r(\chi)$. 
PROPOSITION 9.1. With the above-introduced notation, the following statements hold:

(i) Suppose that $l=\mathrm{r}(\chi) \geqslant k$ for some $\chi \in \operatorname{Irr}\left(\mathrm{GL}_{n}(q)\right)$. Then for any $j$ with $k \leqslant j \leqslant n / 2$, there is an irreducible constituent $\mu$ of $\left.\chi\right|_{U_{j}}$ with $k \leqslant \mathrm{r}(\mu) \leqslant$ $\min (j, l)$.

(ii) Suppose that $\mathrm{r}(\chi)=k$ and $\mathrm{r}(\theta)=l$ for $\mathrm{GL}_{n}(q)$-characters $\chi, \theta$ and that $k+l \leqslant n / 2$. Then $\mathrm{r}(\chi \theta)=k+l$.

(iii) Suppose that $\alpha \in \operatorname{Irr}\left(\mathrm{GL}_{j}(q)\right)$ and $\beta \in \operatorname{Irr}\left(\mathrm{GL}_{n-j}(q)\right)$ with $\mathrm{r}(\alpha)=k$ and $\mathrm{r}(\beta)=$ l. View $\alpha \otimes \beta$ as a character of a Levi subgroup $L_{j}=\operatorname{GL}_{j}(q) \times$ $\mathrm{GL}_{n-j}(q)$ of $P_{j}$. If $\chi \in \operatorname{Irr}\left(\mathrm{GL}_{n}(q)\right)$ is an irreducible constituent of $R_{L_{j}}^{G}(\alpha \otimes$ $\beta)$, then $\mathrm{r}(\chi) \geqslant k+l$.

Proof. (i) By the definition, there are some $i, l$ with $k \leqslant l \leqslant i \leqslant n / 2$ such that $\left.\chi\right|_{U_{i}}$ contains an irreducible constituent $\lambda=\lambda_{Y}$ with $\operatorname{rank}(Y)=l=\mathrm{r}(\chi)$. Note that $L_{i}$ acts on the constituents of $\left.\chi\right|_{U_{i}}$ via conjugation, and conjugating $\lambda$ by a suitable element in $L_{i}$, we may assume that $Y=\left(\begin{array}{cc}I_{l} & 0 \\ 0 & 0\end{array}\right)$. Now we consider the subspace

$$
\tilde{W}_{j}:=\left\langle e_{1}, e_{2}, \ldots, e_{k}, e_{i+k+1}, e_{i+k+2}, \ldots, e_{i+j}\right\rangle_{\mathbb{F}_{q}},
$$

and its stabilizer $\tilde{P}_{j}=\tilde{U}_{j} \rtimes \tilde{L}_{j}$ with unipotent radical $\tilde{U}_{j}$. Let $Q:=U_{i} \cap \tilde{U}_{j}$ and let $\mu:=\lambda_{Z} \in \operatorname{Irr}\left(\tilde{U}_{j}\right)$ be any irreducible constituent of $\left.\chi\right|_{\tilde{U}_{j}}$ that lies above $\left.\lambda\right|_{Q}$. With the given choice of $Y$, it is straightforward to check that $Z=\left(\begin{array}{c}I_{k} * \\ * *\end{array}\right)$, and so $\operatorname{rank}(Z) \geqslant k$. On the other hand, as $\tilde{U}_{j}$ is $G$-conjugate to $U_{j}$, we have that $\operatorname{rank}(Z) \leqslant \mathrm{r}(\chi)=l$, and $\operatorname{rank}(Z) \leqslant j$ as $Z$ is an $(n-j) \times j$-matrix.

(ii) By (i), we may assume that, for $j:=k+l,\left.\chi\right|_{U_{j}}$ contains an irreducible constituent $\lambda=\lambda_{Y}$ of rank $k$ and $\left.\theta\right|_{U_{j}}$ contains an irreducible constituent $\mu=\lambda_{Z}$ of rank $l$. Again conjugating $\lambda$ and $\mu$ by a suitable element in $L_{j}$, we may assume that

$$
Y=\left(\begin{array}{lll}
I_{k} & 0 & 0 \\
0 & 0 & 0
\end{array}\right), \quad Z=\left(\begin{array}{lll}
0 & 0 & 0 \\
0 & I_{l} & 0
\end{array}\right) .
$$

It follows that $\left.(\chi \theta)\right|_{U_{j}}$ contains $\lambda \mu=\lambda_{Y+Z}$ with $\operatorname{rank}(Y+Z)=k+l$. Since the upper bound $r(\chi \theta) \leqslant k+l$ is obvious, the statement follows.

(iii) We identify $\mathrm{GL}_{j}(q)$ and $\mathrm{GL}_{n-j}(q)$ with

$$
\begin{aligned}
G_{1} & :=\operatorname{Stab}_{G}\left(\left\langle e_{1}, \ldots, e_{j}\right\rangle_{\mathbb{F}_{q}}, e_{j+1}, \ldots, e_{n}\right), \\
G_{2} & :=\operatorname{Stab}_{G}\left(e_{1}, \ldots, e_{j},\left\langle e_{j+1}, \ldots, e_{n}\right\rangle_{\mathbb{F}_{q}}\right),
\end{aligned}
$$

respectively. Let $U_{1, k}$ denote the unipotent radical of $\operatorname{Stab}_{G_{1}}\left(\left\langle e_{1}, \ldots, e_{k}\right\rangle_{\mathbb{F}_{q}}\right)$, and let $U_{2, l}$ denote the unipotent radical of $\operatorname{Stab}_{G_{2}}\left(\left\langle e_{j+1}, \ldots, e_{j+l}\right\rangle_{\mathbb{F}_{q}}\right)$. By (i), we may 
assume that $\left.\alpha\right|_{U_{1, k}}$ contains an irreducible constituent $\lambda=\lambda_{X}$ of rank $k$, and after a suitable conjugation, $X=\left(I_{k} 0\right)$. Likewise, we may assume that $\left.\beta\right|_{U_{2, l}}$ contains an irreducible constituent $\mu=\lambda_{Y}$ of rank $l$ with $Y=\left(I_{l} 0\right)$. By the assumption, ${ }^{*} R_{L_{j}}^{G}(\chi)$ contains $\alpha \otimes \beta$, and so the restriction of $\chi$ to $U_{j} \rtimes\left(U_{1, k} \times U_{2, l}\right)$ contains an irreducible constituent $\gamma$ that is trivial on $U_{j}$, equal to $\lambda$ on $U_{1, k}$, and equal to $\mu$ on $U_{2, l}$. Now we consider the subspace

$$
\tilde{W}_{k+l}:=\left\langle e_{1}, e_{2}, \ldots, e_{k}, e_{j+1}, e_{j+2}, \ldots, e_{j+l}\right\rangle_{\mathbb{F}_{q}},
$$

and note that the unipotent radical $\tilde{U}_{k+l}$ of its stabilizer in $G$ contains

$$
Q:=\left(U_{j} \cap \tilde{U}_{k+l}\right) \rtimes\left(U_{1, k} \times U_{2, l}\right) .
$$

Let $\delta:=\lambda_{Z} \in \operatorname{Irr}\left(\tilde{U}_{k+l}\right)$ be any irreducible constituent of $\left.\chi\right|_{\tilde{U}_{k+l}}$ that lies above $\left.\gamma\right|_{Q}$. The conditions on $X, Y$, and $\gamma$ now imply that $Z=\left(\begin{array}{cccc}I_{k} & 0 & Z_{1} & Z_{2} \\ 0 & 0 & I_{l} & 0\end{array}\right)$. Clearly, $\operatorname{rank}(Z)=k+l$, and so $r(\chi) \geqslant k+l$ (since $\tilde{U}_{k+l}$ is again $G$-conjugate to $U_{k+l}$ ).

COROLlary 9.2. For any $\chi \in \operatorname{Irr}\left(\mathrm{GL}_{n}(q)\right), \mathrm{r}(\chi) \leqslant \min (\mathfrak{l}(\chi), n / 2)$.

Proof. The inequality $\mathrm{r}(\chi) \leqslant n / 2$ is immediate by the definition. Also note that $r(\chi)$ does not change if we multiply $\chi$ by a linear character of $G$. Hence, we may assume that $\mathfrak{l}^{*}(\chi)=\mathfrak{l}(\chi)=j \leqslant n / 2$, and so $\chi$ is a constituent of $\left(\tau_{n}\right)^{j}$ by Definition 1. If $2 \leqslant n \leqslant 3$, then $j=1 \leqslant \mathrm{r}(\chi)<3 / 2$ and we are done. So we may assume $n \geqslant 4$ and conclude by [T2, Corollary 2.3] that $\mathrm{r}\left(\tau_{n}\right)=1$. It then follows by Proposition 9.1(ii) that $\mathrm{r}(\chi) \leqslant \mathrm{r}\left(\left(\tau_{n}\right)^{j}\right) \leqslant j$.

We will also need the following result.

LEMMA 9.3. For integers $m \geqslant 2$ and $1 \leqslant j \leqslant m-1$, let $N(m, j)$ denote the number of matrices $X \in M_{m}\left(\mathbb{F}_{q}\right)$ of rank $j$, and let $N^{\prime}(m, j)$ denote the number of those matrices but with the $(1,1)$-entry $X_{11}$ equal to 0 . Then $N^{\prime}(m, j)>$ $N(m, j) / q$.

Proof. Let $Y$ be a uniformly distributed random variable with values in the set $M_{m}\left(\mathbb{F}_{q}\right)$, let $Y^{(1)}$ denote the first column, and let $Y_{1}^{(1)}$ denote the upper left entry. If $\operatorname{Pr}[E]$ denotes the probability of the event $E$, then our claim is that

$$
\operatorname{Pr}\left[\operatorname{rank}(Y)=j \mid Y_{1}^{(1)}=0\right]>\operatorname{Pr}[\operatorname{rank}(Y)=j] .
$$

Now,

$$
\operatorname{Pr}\left[\operatorname{rank}(Y)=j \mid Y_{1}^{(1)}=0\right]
$$




$$
\begin{aligned}
= & \operatorname{Pr}\left[\operatorname{rank}(Y)=j \mid Y^{(1)}=0\right] \operatorname{Pr}\left[Y^{(1)}=0 \mid Y_{1}^{(1)}=0\right] \\
& +\operatorname{Pr}\left[\operatorname{rank}(Y)=j \mid Y^{(1)}=0\right]\left(1-\operatorname{Pr}\left[Y^{(1)}=0 \mid Y_{1}^{(1)}=0\right]\right),
\end{aligned}
$$

while

$$
\begin{aligned}
\operatorname{Pr}[\operatorname{rank}(Y)=j]= & \operatorname{Pr}\left[\operatorname{rank}(Y)=j \mid Y^{(1)}=0\right] \operatorname{Pr}\left[Y^{(1)}=0\right] \\
& +\operatorname{Pr}\left[\operatorname{rank}(Y)=j \mid Y^{(1)} \neq 0\right]\left(1-\operatorname{Pr}\left[Y^{(1)}=0\right]\right) .
\end{aligned}
$$

As

$$
\operatorname{Pr}\left[Y^{(1)}=0 \mid Y_{1}^{(1)}=0\right]>\operatorname{Pr}\left[Y^{(1)}=0\right],
$$

it suffices to show that

$$
\operatorname{Pr}\left[\operatorname{rank}(Y)=j \mid Y^{(1)}=0\right]>\operatorname{Pr}\left[\operatorname{rank}(Y)=j \mid Y^{(1)} \neq 0\right],
$$

or, equivalently,

$$
\operatorname{Pr}\left[\operatorname{rank}(Y)=j \mid Y^{(1)}=0\right]>\operatorname{Pr}[\operatorname{rank}(Y)=j] .
$$

More generally, let $p_{m, n}(j)$ denote the probability $\operatorname{Pr}[\operatorname{rank}(Y)=j]$ for $m \times n$ matrices $Y$ over $\mathbb{F}_{q}$. Then our assertion reduces to the statement that

$$
p_{m, n}(j)<p_{m, n-1}(j)
$$

when $j<\min (m, n)$.

Consider the action of $\mathrm{GL}_{m}(q) \times \mathrm{GL}_{n}(q)$ on $M_{m \times n}\left(\mathbb{F}_{q}\right)$ via $(g, h) \circ X=g X h^{-1}$. As $\Gamma$ acts transitively on the set $\Omega(m, n, j)$ of all $Y \in M_{m \times n}\left(\mathbb{F}_{q}\right)$ of rank $j$, by computing the stabilizer of $Y=\left(\begin{array}{cc}I_{j} & 0 \\ 0 & 0\end{array}\right)$ in $\mathrm{GL}_{m}(q) \times \mathrm{GL}_{n}(q)$, we see that

$$
|\Omega(m, n, j)|=\frac{\prod_{i=0}^{j-1}\left(q^{m}-q^{i}\right) \cdot \prod_{i=0}^{j-1}\left(q^{n}-q^{i}\right)}{\left|\mathrm{GL}_{j}(q)\right|} .
$$

Since $j<\min (m, n)$, it follows that

$$
\frac{p_{m, n}(j)}{p_{m, n-1}(j)}=\frac{|\Omega(m, n, j)| / q^{m n}}{|\Omega(m, n-1, j)| / q^{m(n-1)}}=\frac{q^{n}-1}{q^{m}\left(q^{n-j}-1\right)}<1,
$$

as required.

Recall the notation (3.12). Using Lemma 9.3, we now show

Corollary 9.4. Let $\chi=S(s, \lambda) \in \operatorname{Irr}\left(\mathrm{GL}_{n}(q)\right)$ with $\operatorname{deg}(s)=\left[\mathbb{F}_{q}(s): \mathbb{F}_{q}\right]=$ $d \geqslant 2$. Then $\mathrm{r}(\chi)=\lfloor n / 2\rfloor$. 
Proof. Assume the contrary and let $t \in \mathrm{GL}_{n}(q)$ be a transvection. Put $t$ in the unipotent radical $U_{1}$ of $P_{1}$. By [BDK, Corollary 5.4d(ii)], ${ }^{*} R_{L_{1}}^{G}(\chi)=0$ for a Levi subgroup $L_{1}$ of $P_{1}$. Since $L_{1}$ acts transitively on $\operatorname{Irr}\left(U_{1}\right) \backslash\left\{1_{U_{1}}\right\}$, it follows that $\left.\chi\right|_{U_{1}}$ is a multiple of the sum of all nontrivial linear characters of $U_{1}$, and so $\chi(t)=-\chi(1) /\left(q^{n-1}-1\right)<0$.

First, we consider the case $n=2 m \in 2 \mathbb{Z}$ and take $t=\left(\begin{array}{cc}I_{m} & Y \\ 0 & 0\end{array}\right)$ in the unipotent radical $U_{m}$ of $P_{m}$, with $Y=\operatorname{diag}(1,0, \ldots, 0)$. By the assumption, $\left.\chi\right|_{U_{m}}=$ $\sum_{j=0}^{m-1} n_{j} \Sigma_{j}$, where $n_{j} \in \mathbb{Z}_{\geqslant 0}$ and $\Sigma_{j}:=\sum_{\lambda \in \mathcal{O}_{j}} \lambda, 0 \leqslant j<m$. Here, $\mathcal{O}_{j}$ is the $P_{m}$-orbit of all rank $j$ characters $\lambda=\lambda_{X}$ of $U_{m}$. In the notation of Lemma 9.3, it is easy to see that $\lambda_{X}(t)=1$ if $X_{11}=0$, whereas $\sum_{\alpha \in \mathbb{F}_{q}^{\times}} \lambda_{\alpha X}(t)=-1$. It follows by Lemma 9.3

$$
\Sigma_{j}(t)=N^{\prime}(m, j)-\frac{N(m, j)-N^{\prime}(m, j)}{q-1}=\frac{q N^{\prime}(m, j)-N(m, j)}{q-1}>0,
$$

for all $0 \leqslant j<m$, and so $\chi(t) \geqslant 0$, a contradiction.

Now let $n=2 m+1 \geqslant 3$ be odd. Embed $H:=\mathrm{GL}_{2 m}(q)$ in $G=\mathrm{GL}_{n}(q)$ via $h \mapsto \operatorname{diag}(h, 1)$. Since $\chi(t)<0$, there is some irreducible constituent $\psi$ of $\left.\chi\right|_{H}$ with $\psi(t)<0$. By the previous result, $\mathrm{r}(\psi)=m$. It is easy to see that this implies $r(\chi) \geqslant m$, and so equality holds since $r(\chi) \leqslant n / 2$.

The main result of this subsection is the following theorem, resolving [GH2, Conjecture 6.3.5] and generalizing the complex case of the main result of [T2].

THEOREM 9.5. For any $\chi \in \operatorname{Irr}\left(\mathrm{GL}_{n}(q)\right)$, the $U$-rank $\mathrm{r}(\chi)$ is given by the formula

$$
r(\chi)=\min (\mathfrak{l}(\chi),\lfloor n / 2\rfloor) .
$$

In particular, $\mathfrak{l}(\chi) \geqslant \mathrm{r}(\chi) \geqslant\lfloor\mathfrak{l}(\chi) / 2\rfloor$.

Proof. Let $\mathfrak{l}(\chi)=j$. The case $j=0$ is obvious, so we may assume that $1 \leqslant j \leqslant$ $n$.

(a) First, we prove by induction on $j$ that if $1 \leqslant j \leqslant n / 2$, then $\mathrm{r}(\chi)=\mathfrak{l}(\chi)$. Multiplying $\chi$ by a suitable linear character of $G$, we may assume that $\chi$ is an irreducible constituent of $\left(\tau_{n}\right)^{j}$. Now if $j=1$, then again by [T2, Corollary 2.3], we have that $0<\mathrm{r}(\chi) \leqslant \mathrm{r}\left(\tau_{n}\right)=1$ and $\operatorname{so} \mathrm{r}(\chi)=1$.

For the induction step, we assume $2 \leqslant j \leqslant n / 2$. Fix a basis $\left(e_{1}, \ldots, e_{n}\right)$ of $A$ and a basis $\left(f_{1}, \ldots, f_{j}\right)$ of $B=\mathbb{F}_{q}^{j}$, and consider $v_{0}:=\sum_{i=1}^{j} e_{i} \otimes f_{i} \in$ $V=A \otimes_{\mathbb{F}_{q}} B$. Let $\rho$ denote the permutation character of $G \times S$ acting on $\Omega$, the set of vectors in $V$ of rank $j$, which contains $v_{0}$. As noted in Section 9.1, 
$(g, s) \in \Gamma:=G \times S$ fixes $v_{0}$ exactly when

$$
g=\left(\begin{array}{cc}
{ }^{t} X^{-1} & * \\
0 & Y
\end{array}\right), \quad s=X, X \in \mathrm{GL}_{j}(q), Y \in \mathrm{GL}_{n-j}(q)
$$

(in the chosen bases). Hence, $\operatorname{Stab}_{\tilde{U} \times S}\left(v_{0}\right)=1$, where $\tilde{U}$ is the unipotent radical of

$$
\tilde{P}:=\operatorname{Stab}_{G}\left(\left\langle e_{j+1}, e_{j+2}, \ldots, e_{n}\right\rangle_{\mathbb{F}_{q}}\right) .
$$

Thus, $\tilde{U} \times S$ has a regular orbit on $\Omega$. Since $\left.\tau\right|_{\tilde{U} \times S}$ contains $\left.\rho\right|_{\tilde{U} \times S}$, it follows that $\left.\tau\right|_{\tilde{U} \times S}$ contains the regular character

$$
\operatorname{reg}_{\tilde{U} \times S}=\sum_{\alpha \in \operatorname{Irr}(S), \lambda \in \operatorname{Irr}(\tilde{U})} \alpha(1) \lambda \otimes \alpha .
$$

Hence, for any $\alpha \in \operatorname{Irr}(S)$ and the $G$-character $D_{\alpha}$ defined in Theorem 1.1(ii), we have that $\left.D_{\alpha}\right|_{\tilde{U}}$ contains $\alpha(1) \mathbf{r e g}_{\tilde{U}}$.

By Theorem 1.1(ii), we may assume that $\chi=D_{\alpha}^{\circ}$ for some $\alpha \in \operatorname{Irr}(S)$. Next, let $\sigma$ denote the transpose-inverse automorphism of $G$ (defined in the given basis of $A$ ). Arguing as in (8.3), we see that $\sigma$ acts on any $G$-character via complex conjugation. Now observe that $\sigma$ sends $\tilde{P}$ to $P_{j}=\operatorname{Stab}\left(\left\langle e_{1}, \ldots, e_{j}\right\rangle_{\mathbb{F}_{q}}\right)$ and $\tilde{U}$ to the unipotent radical $U_{j}$ of $P_{j}$. So the result of the preceding paragraph, applied to $D_{\bar{\alpha}}$, implies that $\left.D_{\alpha}\right|_{U_{j}}$ contains $\alpha(1) \operatorname{reg}_{U_{j}}$. In particular, if $\lambda \in \operatorname{Irr}\left(U_{j}\right)$ has rank $j$ (recall $j \leqslant n / 2$ ), then $\left.D_{\alpha}\right|_{U_{j}}$ contains $\alpha(1) \lambda$. On the other hand, $\operatorname{r}\left(D_{\alpha}-\right.$ $\left.D_{\alpha}^{\circ}\right)<j$ by the induction hypothesis and Theorem 1.1(ii). It follows that $\chi=D_{\alpha}^{\circ}$ contains $\alpha(1) \lambda$, whence $r(\chi)=j$ by Corollary 9.2.

In fact, observe that for any $g \in U_{j}, \tau_{n}(g) \in\left\{q^{n}, q^{n-1}, \ldots, q^{n-j}\right\}$, and $\tau_{n}(g)=$ $q^{n}$ if and only if $g=1$. Hence, for $\psi:=\prod_{i=n-j}^{n-1}\left(\tau_{n}-q^{i} \cdot 1_{G}\right)$, we have that $\left.\psi\right|_{U_{j}}=\left|\mathrm{GL}_{j}(q)\right| \cdot \operatorname{reg}_{U_{j}}$. Since $\mathrm{r}\left(\left(\tau_{n}\right)^{i}\right) \leqslant i$, it follows that

$$
\left[\left.\left(\left(\tau_{n}\right)^{j}\right)\right|_{U_{j}}, \lambda\right]_{U_{j}}=\left|\mathrm{GL}_{j}(q)\right|=|S|=\sum_{\beta \in \operatorname{Irr}(S)} \beta(1)^{2} .
$$

On the other hand, each $D_{\beta}^{\circ}$ with $\beta \in \operatorname{Irr}(S)$ contains $\beta(1) \lambda$ as shown above. Hence, we have proved that each $U_{j}$-character of rank $j$ has multiplicity exactly $\alpha(1)$ in $\left.\chi\right|_{U_{j}}$ when $\chi=D_{\alpha}^{\circ}$ has true level $j$.

(ii) Now we prove by induction on $n \geqslant 1$ that if $j>n / 2$, then $\mathrm{r}(\chi)=\lfloor n / 2\rfloor$. The induction base $n \leqslant 3$ is obvious. For the induction step, denote $m:=\lfloor n / 2\rfloor$ and assume that the statement holds for $n$ up to $2 m-1$. We will also write $\chi$ in the form (3.12).

Consider the case $\chi=S(s, \lambda)$. If $\operatorname{deg}(s)>1$, then we are done by Corollary 9.4. Hence, we may assume that $s=1$, and so

$$
\lambda=\left(\lambda_{1}=n-j \geqslant \lambda_{2} \geqslant \cdots \geqslant \lambda_{s}>0\right) \vdash n
$$


by Theorem 3.9. The assumption $j>n / 2$ implies that $s \geqslant 2$.

Suppose first that $\lambda$ has repeated rows. Then we look at the last repeated rows of $\lambda$ :

$$
\lambda_{a}=\cdots=\lambda_{a+k}>\lambda_{a+k+1}>\cdots>\lambda_{s}
$$

with $a, k \geqslant 1$ and $a+k \leqslant s$. Then the $\operatorname{rim} R$ of the Young diagram $Y(\lambda)$ contains a 2-hook $H$ consisting of the last two nodes of $\lambda_{a+k-1}$ and $\lambda_{a+k}$. Setting

$$
\begin{gathered}
\boldsymbol{\gamma}:=\left(\lambda_{1}, \ldots, \lambda_{a+k-2}, \lambda_{a+k-1}-1, \lambda_{a+k}-1, \lambda_{a+k+1}, \ldots, \lambda_{s}\right) \vdash(n-2), \\
\delta:=(1,1),
\end{gathered}
$$

we see that $R \backslash H$ is the rim of $Y(\boldsymbol{\gamma})$. Denoting by $\chi^{\lambda}$ the irreducible character of $\mathrm{S}_{n}$ labeled by $\lambda$, and similarly $\chi^{\gamma} \in \operatorname{Irr}\left(\mathrm{S}_{n-2}\right)$ and $\chi^{\delta} \in \operatorname{Irr}\left(\mathrm{S}_{2}\right)$, we see by [GKNT, Lemma 4.1] that

$$
\chi^{\lambda} \mid S_{n-2} \times S_{2} \text { contains } \chi^{\gamma} \otimes \chi^{\delta} .
$$

Suppose now that $\lambda$ has no repeated rows. Removing the last node of the first and the last node of the second row of $Y(\lambda)$ from $R$, we now get the rim of $Y(\gamma)$, with $\gamma:=\left(\lambda_{1}-1, \lambda_{2}-1, \lambda_{3}, \ldots, \lambda_{s}\right) \vdash n-2$. Keeping $\delta:=(1,1)$, we then see by [JK, Corollary 2.8.14] that (9.1) holds in this case as well.

In turn, (9.1) implies by Lemma 3.3 that

$$
{ }^{*} R_{\mathrm{GL}_{n-2}(q) \times \mathrm{GL}_{2}(q)}^{\mathrm{GL}_{n}(\chi)}(\chi) \text { contains } S(1, \boldsymbol{\gamma}) \otimes S(1, \boldsymbol{\delta}) .
$$

Note that $\mathrm{r}(S(1, \boldsymbol{\delta}))=1$. Furthermore, the first part of $\boldsymbol{\gamma}$ is $n-j$ or $n-j-1$, and so $j^{\prime}:=\mathfrak{l}(S(1, \boldsymbol{\gamma})) \geqslant j-2 \geqslant m-1$. It follows by (i) when $j^{\prime}=m-1$ and by the induction hypothesis when $j^{\prime} \geqslant m$ that $\mathrm{r}(S(1, \boldsymbol{\gamma}))=\lfloor(n-2) / 2\rfloor=m-1$. Now applying Proposition 9.1(iii) to (9.2), we obtain that $r(\chi) \geqslant m$, as desired.

(iii) To complete the proof, it remains to consider the case $\chi=R_{L}^{G}(\alpha \otimes \beta)$, where $L=\mathrm{GL}_{a}(q) \times \mathrm{GL}_{b}(q)$ with $1 \leqslant a \leqslant b, \alpha \in \operatorname{Irr}\left(\mathrm{GL}_{a}(q)\right), \beta \in \operatorname{Irr}\left(\mathrm{GL}_{b}(q)\right)$. Here we can take

$$
L=\operatorname{Stab}_{G}\left(\left\langle e_{1}, \ldots, e_{a}\right\rangle_{\mathbb{F}_{q}},\left\langle e_{a+1}, \ldots, e_{n}\right\rangle_{\mathbb{F}_{q}}\right),
$$

a Levi subgroup of $P_{a}=\operatorname{Stab}_{G}\left(\left\langle e_{1}, \ldots, e_{a}\right\rangle_{\mathbb{F}_{q}}\right)$. We also consider a 'middle' parabolic subgroup

$$
\tilde{P}_{m}=\operatorname{Stab}_{G}\left(\left\langle e_{n-m+1}, \ldots, e_{n}\right\rangle_{\mathbb{F}_{q}}\right)
$$

with unipotent radical $Q$. Observe that $Q=R \times T$, where

$$
R:=P_{a} \cap Q=\left\{\left(\begin{array}{ccc}
I_{a} & 0 & 0 \\
0 & I_{b-m} & 0 \\
0 & * & I_{m}
\end{array}\right)\right\}, \quad T:=\left\{\left(\begin{array}{ccc}
I_{a} & 0 & 0 \\
0 & I_{b-m} & 0 \\
* & 0 & I_{m}
\end{array}\right)\right\} .
$$


Furthermore, $R$ is the unipotent radical of the parabolic subgroup

$$
\operatorname{Stab}_{\mathrm{GL}_{b}(q)}\left(\left\langle e_{n-m+1}, \ldots, e_{n}\right\rangle_{\mathbb{F}_{q}}\right)
$$

in the second direct factor of $L$.

The condition $\mathfrak{l}(\chi)=j \geqslant m+1$ implies by Theorem 3.9 that

$$
\mathfrak{l}(\beta) \geqslant b-(n-j)=j-a \geqslant b-m .
$$

As $b-m \leqslant b / 2$, applying (i) and the induction hypothesis to $\operatorname{GL}_{b}(q)$, we conclude that $\mathrm{r}(\beta) \geqslant b-m$. Hence, by Proposition 9.1(i), $\left.\beta\right|_{R}$ contains a character $v=\lambda_{Z}$ of maximum rank $b-m$; in fact, we can take $Z=\left(\begin{array}{ll}0 I_{b-m}\end{array}\right)$.

Recall that $\chi=\operatorname{Ind}_{P_{a}}^{G}(\varphi)$, where $U_{a} \leqslant \operatorname{Ker}(\varphi)$ and $\left.\varphi\right|_{L}=\alpha \otimes \beta$. Therefore, $\left.\chi\right|_{Q}$ contains

$$
\operatorname{Ind}_{P_{a} \cap Q}^{Q}\left(\left.\varphi\right|_{P_{a} \cap Q}\right)=\operatorname{Ind}_{R}^{R T}\left(\left.\varphi\right|_{R}\right)=\operatorname{Ind}_{R}^{R T}\left(\left.\alpha(1) \beta\right|_{R}\right)=\left(\left.\alpha(1) \beta\right|_{R}\right) \mathbf{r e g}_{T} ;
$$

in particular, $\left.\chi\right|_{Q}$ contains $v \cdot \mathbf{r e g}_{T}$. Since $2 m-b \leqslant a$, $\operatorname{reg}_{T}$ contains a character $\mu=\lambda_{Y}$ of rank $2 m-b$; in fact, we can take $Y=\left(\begin{array}{rr}I_{2 m-b} & 0 \\ 0 & 0\end{array}\right)$. Viewing $Q$ as $T \times R$, we see that $\left.\chi\right|_{Q}$ contains $\mu \otimes v=\lambda_{Y} \otimes \lambda_{Z}=\lambda_{X}$ with $X=\left(\begin{array}{l}Y \\ Z\end{array}\right)$. By the construction, $X$ has rank $m$, whence $r(\chi) \geqslant m$, as stated.

Note that Theorem 9.5 shows that [GH2, Proposition 11.1] is false in the case $\mathfrak{l}(\chi)=n=2 m+1$. Indeed, when $n=2 m+1$ we can take $\chi \in \operatorname{Irr}\left(\mathrm{GL}_{n}(q)\right)$ of maximum level $n$, for which we have $r(\chi)=m$, whereas [GH2, Proposition 11.1] states that $\mathfrak{l}(\theta) \leqslant 2 \mathrm{r}(\theta)$ for all $\theta \in \operatorname{Irr}\left(\mathrm{GL}_{2 m+1}(q)\right)$.

9.2. Some final remarks. First, we give an example to show that the exponent $1-1 / n$ in Theorem 1.6 is optimal (in the range where $q$ is bounded and $n \rightarrow \infty)$.

EXAmPLE 9.6. Let $G=\mathrm{GL}(A)=\mathrm{GL}_{n}^{\epsilon}(q)$ for $\epsilon= \pm$ and let $\chi=\chi^{(n-1,1)}$ be the unipotent Weil character of level 1 . Then $\chi(1) \approx q^{n} /(q-\epsilon)$.

(i) $\chi(g) \approx q^{n-1} /(q-\epsilon)$ if $g$ is a transvection.

(ii) For any $n / 2<k \leqslant n-1$, we can find $g \in \operatorname{SL}_{n}^{\epsilon}(q)$ such that the $g$-fixed point subspace on $A$ has dimension $k$ (and so $\delta(g)=d_{A}(g)=k$ ). Then $\chi(g) \approx q^{k} /(q-\epsilon)$.

REMARK 9.7. Let $G=\operatorname{GL}_{n}^{\epsilon}(q)$ for $\epsilon= \pm$ and $S=\operatorname{SL}_{n}^{\epsilon}(q)$. One can define a certain subgroup $D$ of outer automorphisms of $G$ (in a compatible way for all 
$n$ ) such that the action of $G \rtimes D$ on $S$ induces $\operatorname{Aut}(S)$; see [GKNT, (5.2)]. In particular, this gives rise to an action of $D$ on irreducible characters of $G, S$, and $\mathrm{GL}_{j}^{\epsilon}(q)$ with $1 \leqslant j \leqslant n$. Furthermore, $\Gamma:=\operatorname{Gal}(\overline{\mathbb{Q}} / \mathbb{Q})$ also acts naturally on those characters. In the case of $G$ and $\mathrm{GL}_{j}^{\epsilon}(q)$, these actions are well understood; see, for example, [GKNT, Section 5]. Furthermore, $\tau_{n}$, respectively $\zeta_{n}$, is $D$ invariant and $\Gamma$-invariant; in particular, $I^{*}(\chi)$ is preserved under the action of $D$ and $\Gamma$. It is straightforward now to check that the bijection in Corollary 3.10 is $D$-equivariant and $\Gamma$-equivariant. Finally, if $\varphi \in \operatorname{Irr}(S)$ has level $j<n / 2$, then by Corollary 8.6, it lies under a unique $\chi \in \operatorname{Irr}(G)$ of true level $j$. Combined with the $D$-equivariance and $\Gamma$-equivariance in the $\mathrm{GL}^{\epsilon}$-case, this implies that the bijection in Corollary 8.6(ii) is $D$-equivariant and $\Gamma$-equivariant.

Theorem 1.1 exhibits certain irreducible constituents of $\left.\tau\right|_{G \times S}$, namely $D_{\alpha}^{\circ} \otimes \alpha$ with $\alpha \in \operatorname{Irr}(S)$, where $G=\operatorname{GL}_{n}^{\epsilon}(q)$ and $S=\operatorname{GL}_{j}^{\epsilon}(q)$. One may be interested in the total number of irreducible constituents of $\left.\tau\right|_{G \times S}$ or at least $N_{n, j}:=\left[\left.\tau\right|_{G \times S}\right.$, $\left.\left.\tau\right|_{G \times S}\right]_{G \times S}$. We will now provide some upper and lower bounds on the latter invariant.

First, we consider the linear case, and let $S=\mathrm{GL}_{j}(q)=\mathrm{GL}(U), G=$ $\mathrm{GL}_{n}(q)=\mathrm{GL}(W)$ with $1 \leqslant j \leqslant n, U=\mathbb{F}_{q}^{j}, W=\mathbb{F}_{q}^{n}$. Set $V=U \otimes W \cong \mathbb{F}_{q}^{n j}$. As $\tau$ is the permutation character of $\operatorname{GL}(V)$ on the point set of $V, N_{n, j}$ is the number of orbits of $S \times G$ acting on $V \oplus V$. Note that $V$ is $\operatorname{Aut}(S \times G)$-equivalent to $\operatorname{Hom}(U, W)$ with the natural $S \times G$-action. Thus, we are counting orbits on ordered pairs of linear transformations from $U$ to $W$. These were classified by Kronecker (see [Ga], and, for an elementary treatment, see [Sp]).

Now, given a pair $(A, B)$ with $A, B \in \operatorname{Hom}(U, W)$ (or, equivalently, the pencil $A+x B)$, we can replace $W$ by any subspace of $W$ that contains $\operatorname{Im}(A)+\operatorname{Im}(B)$. Hence, the number of orbits $N_{n, j}$ for $n=2 j$ is the same as $N_{n, j}$ for any $n \geqslant 2 j$.

What Kronecker showed is that we can decompose any pencil $A+x B$ as a direct sum. More precisely, we can write $U=\bigoplus_{i} U_{i}$ and $W=\bigoplus_{i} W_{i}$ such that $A\left(U_{i}\right), B\left(U_{i}\right) \subseteq W_{i}$, and one of the following holds:

(i) $d=\operatorname{dim} U_{i}=\operatorname{dim} W_{i}$ and

(a) $\left(\left.A\right|_{U_{i}},\left.B\right|_{U_{i}}\right)=\left(I_{d}, X\right)$, where $X$ invertible;

(b) $\left(\left.A\right|_{U_{i}},\left.B\right|_{U_{i}}\right)=\left(I_{d}, Y\right)$, where $Y$ is nilpotent; or

(c) $\left(\left.A\right|_{U_{i}},\left.B\right|_{U_{i}}\right)=\left(Y, I_{d}\right)$ where $Y$ is nilpotent;

(ii) $\operatorname{dim} U_{i}+1=\operatorname{dim} W_{i} \geqslant 2$ and there is a unique $\left(\left.A\right|_{U_{i}},\left.B\right|_{U_{i}}\right)$ depending only on $\operatorname{dim} U_{i}$;

(iii) $\operatorname{dim} U_{i}=1+\operatorname{dim} W_{i} \geqslant 2$ and there is a unique $\left(\left.A\right|_{U_{i}},\left.B\right|_{U_{i}}\right)$ depending only on $\operatorname{dim} U_{i}$;

(iv) $\left(\left.A\right|_{U_{i}},\left.B\right|_{U_{i}}\right)=(0,0)$. 
Here, in (a), (b), respectively (c), $A$, respectively $B$, is represented by the identity matrix $I_{d}$ in suitable bases of $U_{i}$ and $W_{i}$. Moreover, this decomposition is unique up to the conjugacy of $X$ and $Y$ and the dimensions of the pieces. We can also combine pieces of the same kind (a), (b), (c), or (iv) so that each of these four types occurs for at most one index $i$.

Now assume that the term of type (a) in the decomposition occurs for $r=\operatorname{dim} U_{i}$ (with $0 \leqslant r \leqslant j$ ). The number of orbits on the first part of the decomposition is just $k\left(\mathrm{GL}_{r}(q)\right)$, where $k(H)=|\operatorname{Irr}(H)|$ as usual. For the remainder of the decomposition, we write $j-r=a+b+c+d+e$ as the sum of the totals of $\operatorname{dim} U_{i}$ for each of the remaining five types. Let $p(m)$ denote the number of partitions of $m$, and let $p^{\prime}(m)$ be the number of partitions of $m$ with no parts of size 1 . Then the number of nilpotent classes of $a \times a$-matrices is $p(a)$. Next, the contribution of type (ii), respectively (iii), to $N_{n, j}$ is $p(c)$, respectively $p^{\prime}(d)$. It follows for $n \geqslant 2 j$ that $N_{n, j}=F(j, q)$, where

$$
F(j, q):=\sum_{r=0}^{j} f_{j-r} k\left(\operatorname{GL}_{r}(q)\right),
$$

where

$$
f_{m}:=\sum_{a, b, c, d \in \mathbb{Z} \geqslant 0, a+b+c+d \leqslant m} p(a) p(b) p(c) p^{\prime}(d) .
$$

Note that we have, in fact, shown that $N_{n, j} \leqslant F(j, q)$ for any $1 \leqslant j \leqslant n$. If $j \geqslant n / 2$, to find $N_{n, j}$ precisely, the only extra condition required is that we have to see that $\sum_{i} \operatorname{dim} W_{i}$ in the decomposition is at most $n$. One can easily write down the exact formula. We just note that by ignoring the pieces of type (ii) (where $\operatorname{dim} W_{i}>\operatorname{dim} U_{i}$ ), we obtain the following lower bound:

$$
N_{n, j} \geqslant \sum_{r=0}^{j} h_{j-r} k\left(\mathrm{GL}_{r}(q)\right) \geqslant \sum_{r=0}^{j} k\left(\mathrm{GL}_{r}(q)\right),
$$

where

$$
h_{m}:=\sum_{a, b, d \in \mathbb{Z} \geqslant 0, a+b+d \leqslant m} p(a) p(b) p^{\prime}(d) .
$$

Next, we consider the unitary case and let $S=\mathrm{GU}_{j}(q)=\mathrm{GU}(U), G=$ $\mathrm{GU}_{n}(q)=\mathrm{GU}(W)$ with $1 \leqslant j \leqslant n, U=\mathbb{F}_{q^{2}}^{j}, W=\mathbb{F}_{q^{2}}^{n}$. Set $V=U \otimes W \cong \mathbb{F}_{q^{2}}^{n j}$. As $\tau^{2}$ is now the permutation character of $\mathrm{GU}(V)$ on the point set of $V, N_{n, j}$ is the number of orbits of $S \times G$ acting on $V$. We again replace $V$ by $\operatorname{Hom}(U, W)$, and note a couple of easy facts.

We may assume that $n \leqslant 2 j$, for we can replace $W$ by any nondegenerate subspace containing the image of $T \in \operatorname{Hom}(U, W)$. Suppose that $\operatorname{Ker}(T)=0$ 
and $\operatorname{Im}(T)$ is nondegenerate. Then $T^{*} T$, with $T^{*}={ }^{t} T^{(q)}=\left(x_{s r}^{q}\right)$ for $T=\left(x_{r s}\right)$, is an invertible Hermitian operator on $U$ and the $S$-conjugacy class of the latter is an invariant for the $S \times G$-orbit of $T$. Conversely, if $M$ is any invertible Hermitian $j \times j$-matrix over $\mathbb{F}_{q^{2}}$, then the corresponding Hermitian form has Gram matrix $I_{j}$ in a suitable basis of $U$ and so $M=T^{*} T$ for a suitable injective $T$ with nondegenerate image. Also note that $M={ }^{t} M^{(q)}$ is $\mathrm{GL}_{j}\left(\overline{\mathbb{F}}_{q}\right)$-conjugate to $M^{(q)}$ and so, by the Lang-Steinberg theorem, $M$ is $\mathrm{GL}_{j}\left(\overline{\mathbb{F}}_{q}\right)$-conjugate to some $M^{\prime} \in$ $\mathrm{GL}_{j}(q)$. By [TaZ, Theorem 1], ${ }^{t} M^{\prime}=A M A^{-1}$ for some symmetric $A \in \mathrm{GL}_{j}(q)$, and so $M^{\prime}$ is self-adjoint with respect to the Hermitian form with Gram matrix $A$. Finally, two elements of $\mathrm{GL}_{j}(q)$ are $\mathrm{GL}_{j}(q)$-conjugate precisely when they are $\mathrm{GL}_{j}\left(\overline{\mathbb{F}}_{q}\right)$-conjugate, again by the Lang-Steinberg theorem. We have, therefore, shown that the number of $\mathrm{GU}_{j}(q)$-conjugacy classes of invertible Hermitian $j \times j$-matrices over $\mathbb{F}_{q^{2}}$ is at least $k\left(\mathrm{GL}_{j}(q)\right)$ (in fact, equality holds; see [FG2, Lemma 3.1]).

We can do the same thing for $T$ such that both $\operatorname{Ker}(T)$ and $\operatorname{Im}(T)$ are nondegenerate. Thus, we obtain the lower bound $N_{n, j} \geqslant \sum_{r=0}^{j} k\left(\mathrm{GL}_{r}(q)\right)$.

For $1 \leqslant j \leqslant n / 2$, we can prove another lower bound for $N_{n, j}$. Note that $\left.\tau\right|_{G}=\left(\zeta_{n}\right)^{j}$ contains $\left(\zeta_{n}\right)^{j-2}$ since $\left(\zeta_{n}\right)^{2}$ is the permutation character of $G$ on the point set of $W$. It follows that $\left.\tau\right|_{G}$ contains all irreducible characters of true level $j-2 i, 0 \leqslant i \leqslant j / 2$. Hence, Theorem 1.1(i) implies the lower bound

$$
N_{n, j} \geqslant \sum_{0 \leqslant i \leqslant j / 2} k\left(\mathrm{GU}_{j-2 i}(q)\right) .
$$

We summarize our results in the following statement.

PROPOSITION 9.8. In the notation of Theorem 1.1, let $N_{n, j}:=\left[\left.\tau\right|_{G \times S}\right.$, $\left.\left.\tau\right|_{G \times S}\right]_{G \times S}$. Then

$$
N_{n, j} \geqslant \sum_{r=0}^{j} k\left(\mathrm{GL}_{r}(q)\right) .
$$

In fact, in the linear case, that is when $(G, S)=\left(\mathrm{GL}_{n}(q), \mathrm{GL}_{j}(q)\right)$, we have

$$
\sum_{r=0}^{j} h_{j-r} k\left(\mathrm{GL}_{r}(q)\right) \leqslant N_{n, j} \leqslant \sum_{r=0}^{j} f_{j-r} k\left(\mathrm{GL}_{r}(q)\right)
$$

with $f_{m}$ and $h_{m}$ as defined in (9.4) and (9.5). In the unitary case, if $1 \leqslant j \leqslant n / 2$, then

$$
N_{n, j} \geqslant \sum_{0 \leqslant i \leqslant j / 2} k\left(\mathrm{GU}_{j-2 i}(q)\right)
$$


In fact, using the Lang-Steinberg theorem, one can show that the function $F(j, q)$ defined in (9.3) also gives an upper bound for $N_{n, j}$ in the unitary case (for a detailed argument see $[\mathrm{Gu}]$ ).

\section{Acknowledgements}

Robert Guralnick was partially supported by NSF grants DMS-1600056 and DMS-1901595. Michael Larsen was partially supported by NSF grants DMS1401419 and DMS-1702152. Pham Tiep was partially supported by NSF grants DMS-1201374 and DMS-1840702, the Simons Foundation Fellowship 305247, the Joshua Barlaz Chair in mathematics, and a Clay Senior Scholarship. The authors are grateful to the referee for the careful reading and many helpful comments that greatly improved the exposition of the paper.

Conflict of Interest: There are no conflicts of interest.

\section{References}

[AH] Z. Arad and M. Herzog (Eds.), Products of Conjugacy Classes in Groups, Springer Lecture Notes, 1112 (Springer, Berlin, 1985).

[A] M. Aschbacher, Finite Group Theory, 2nd edn, Cambridge Studies in Advanced Mathematics, 10 (Cambridge University Press, Cambridge, 2000).

[BLST] R. Bezrukavnikov, M. W. Liebeck, A. Shalev and P. H. Tiep, 'Character bounds for finite groups of Lie type', Acta Math. 221 (2018), 1-57.

[BDK] J. Brundan, R. Dipper and A. S. Kleshchev, 'Quantum linear groups and representations of $\mathrm{GL}_{n}\left(\mathbb{F}_{q}\right)$ ', Mem. Amer. Math. Soc. 149(706) (2001).

[C] R. Carter, Finite Groups of Lie type: Conjugacy Classes and Complex Characters, (Wiley, Chichester, 1985).

[DS] P. Diaconis and M. Shahshahani, 'Generating a random permutation with random transpositions', Z. Wahrsch. Verw. Gebiete 57 (1981), 159-179.

[DM1] F. Digne and J. Michel, 'Foncteurs de Lusztig et caractères des groupes linéaires et unitaires sur un corps fini', J. Algebra 107 (1987), 217-255.

[DM2] F. Digne and J. Michel, Representations of Finite Groups of Lie Type, London Mathematical Society Student Texts, 21 (Cambridge University Press, Cambridge, 1991).

[E] V. Ennola, 'On the characters of the finite unitary groups', Ann. Acad. Sci. Fenn. Ser. A I(323) (1963), 35 pages.

[FS] P. Fong and B. Srinivasan, 'The blocks of finite general and unitary groups', Invent. Math. 69 (1982), 109-153.

[FG1] J. Fulman and R. M. Guralnick, 'Bounds on the number and sizes of conjugacy classes in finite Chevalley groups with applications to derangements', Trans. Amer. Math. Soc. 364 (2012), 3023-3070.

[FG2] J. Fulman and R. M. Guralnick, 'Enumeration of commuting pairs in Lie algebras over finite fields', Ann. Comb. 22 (2018), 295-316. 
[Ga] F. R. Gantmacher, Matrix Theory, Vol. 2 (Chelsea, New York, 1977).

[Geck] M. Geck, 'On the average values of the irreducible characters of finite groups of Lie type on geometric unipotent classes', Doc. Math. 1 (1996), 293-317.

[GM] M. Geck and G. Malle, 'On the existence of a unipotent support for the irreducible characters of a finite group of Lie type', Trans. Amer. Math. Soc. 352 (2000), 429-456.

[GP] M. Geck and G. Pfeiffer, Characters of Finite Coxeter Groups and IwahoriHecke Algebras, London Mathematical Society Monographs (Oxford University Press, Oxford, 2000).

[Ge] P. Gérardin, 'Weil representations associated to finite fields', J. Algebra 46 (1977), 54-101.

[GKNT] E. Giannelli, A. S. Kleshchev, G. Navarro and P. H. Tiep, 'Restriction of odd degree characters and natural correspondences', Int. Math. Res. Not. IMRN 2017(20) (2017), 6089-6118.

[Gr] B. H. Gross, 'Group representations and lattices', J. Amer. Math. Soc. 3 (1990), 929-960.

[Gu] R. M. Guralnick, 'On the singular value decomposition over finite fields and orbits of $G U \times G U^{\prime}$, Preprint, 2018, arXiv:1805.06999.

[GLT] R. M. Guralnick, M. Larsen and P. H. Tiep, 'Character levels and character bounds for finite classical groups', Preprint, 2019, arXiv:1904.08070.

[GMST] R. M. Guralnick, K. Magaard, J. Saxl and P. H. Tiep, 'Cross characteristic representations of symplectic groups and unitary groups', J. Algebra 257 (2002), 291-347. Addendum, J. Algebra 299 (2006), 443-446.

[GT] R. M. Guralnick and P. H. Tiep, 'Cross characteristic representations of even characteristic symplectic groups', Trans. Amer. Math. Soc. 356 (2004), 4969-5023.

[GH1] S. Gurevich and R. Howe, 'Small representations of finite classical groups', in Representation Theory, Number Theory, and Invariant Theory, Progress in Mathematics, 323 (Birkhäuser/Springer, Cham, 2017), 209-234.

[GH2] S. Gurevich and R. Howe, 'Rank and duality in representation theory', Preprint.

[Hw] R. E. Howe, 'On the characters of Weil's representations', Trans. Amer. Math. Soc. 177 (1973), 287-298.

[Is] I. M. Isaacs, Character Theory of Finite Groups (AMS-Chelsea, Providence, 2006).

[J1] G. James, 'The irreducible representations of the finite general linear groups', Proc. Lond. Math. Soc. (3) 52 (1986), 236-268.

[J2] G. James, 'The decomposition matrices of $\mathrm{GL}_{n}(q)$ for $n \leqslant 10$ ', Proc. Lond. Math. Soc. (3) 60 (1990), 225-265.

[JK] G. James and A. Kerber, The Representation Theory of the Symmetric Group, Encyclopedia of Mathematics and its Applications, 16 (Addison-Wesley Publishing Co., Reading, MA, 1981).

[K] N. Kawanaka, 'Generalized Gelfand-Graev representations of exceptional simple algebraic groups over a finite field. I', Invent. Math. 84 (1986), 575-616.

[KT1] A. S. Kleshchev and P. H. Tiep, 'Representations of finite special linear groups in nondefining characteristic', Adv. Math. 220 (2009), 478-504.

[KT2] A. S. Kleshchev and P. H. Tiep, 'Representations of general linear groups which are irreducible over subgroups', Amer. J. Math. 132 (2010), 425-473.

[Ku] B. A. Kupershmidt, ' $q$-Newton binomial: from Euler to Gauss', J. Nonlinear Math. Phys. 7 (2000), 244-262.

[LMT] M. Larsen, G. Malle and P. H. Tiep, 'The largest irreducible representations of simple groups', Proc. Lond. Math. Soc. (3) 106 (2013), 65-96. 
[LST] M. Larsen, A. Shalev and P. H. Tiep, 'Probabilistic Waring problems for finite simple groups', Ann. of Math. (2) 190 (2019), 561-608.

[LBST1] M. W. Liebeck, E. A. O'Brien, A. Shalev and P. H. Tiep, 'The Ore conjecture', J. Eur. Math. Soc. (JEMS) 12 (2010), 939-1008.

[LBST2] M. W. Liebeck, E. A. O'Brien, A. Shalev and P. H. Tiep, 'Products of squares in finite simple groups', Proc. Amer. Math. Soc. 140 (2012), 21-33.

[LS] M. W. Liebeck and A. Shalev, 'Character degrees and random walks in finite groups of Lie type', Proc. Lond. Math. Soc. (3) 90 (2005), 61-86.

[L] G. Lusztig, Characters of Reductive Groups over a Finite Field, Annals of Mathematics Studies, 107 (Princeton Univ. Press, Princeton, 1984).

[M] I. G. Macdonald, Symmetric Functions and Hall Polynomials, 2nd edn, Oxford Mathematical Monographs (Oxford University Press, New York, 1995), with contributions by A. Zelevinsky.

[Ma] G. Malle, 'Unipotente Grade imprimitiver komplexer Spiegelungsgruppen', J. Algebra 177 (1995), 768-826.

[Ol] J. B. Olsson, 'Remarks on symbols, hooks and degrees of unipotent characters', J. Combin. Theory Ser. A 42 (1986), 223-238.

[Sp] C. de Seguins Pazzis, 'Invariance of simultaneous similarity and equivalence of matrices under extension of the ground field', Linear Algebra Appl. 433 (2010), $618-624$.

[Sh] A. Shalev, 'Commutators, words, conjugacy classes and character methods', Turk. J. Math. 31 (2007), 131-148.

[ST] A. Shalev and P. H. Tiep, 'Some conjectures on Frobenius' character sum', Bull. Lond. Math. Soc. 49 (2017), 895-902.

[S1] B. Srinivasan, 'The modular representation ring of a cyclic p-group', Proc. Lond. Math. Soc. (3) 14 (1974), 677-688.

[S2] B. Srinivasan, 'Weil representations of finite classical groups', Invent. Math. 51 (1979), 143-153.

[TaZ] O. Taussky and H. Zassenhaus, 'On the similarity transformation between a matrix and its transpose', Pacific J. Math. 9 (1959), 893-896.

[TT] J. Taylor and P. H. Tiep, 'Lusztig induction, unipotent support, and character bounds', Preprint, 2018, arXiv:1809.00173.

[ThV] N. Thiem and C. R. Vinroot, 'On the characteristic map of finite unitary groups', $A d v$. Math. 210 (2007), 707-732.

[T1] P. H. Tiep, 'Dual pairs of finite classical groups in cross characteristic', Contemp. Math. 524 (2010), 161-179.

[T2] P. H. Tiep, 'Weil representations of finite general linear groups and finite special linear groups', Pacific J. Math. 279 (2015), 481-498.

[TZ] P. H. Tiep and A. E. Zalesskii, 'Some characterizations of the Weil representations of the symplectic and unitary groups', J. Algebra 192 (1997), 130-165.

[W] A. Weil, 'Sur certains groupes d'opérateurs unitaires', Acta Math. 111 (1964), 143-211. 